Medidas DLR e Transições de Fase Tipo Volume em Shifts de Markov com Alfabeto Enumerável

Elmer Rusbert Calderón Beltrán 



\title{
Medidas DLR e Transições de Fase Tipo Volume em Shifts de Markov com Alfabeto Enumerável
}

\author{
Elmer Rusbert Calderón Beltrán
}

TESE APRESENTADA

$\mathrm{AO}$

INSTITUTO DE MATEMÁTICA E ESTATÍSTICA

DA

UNIVERSIDADE DE SÃO PAULO

PARA

OBTENÇÃO DO TÍTULO

$\mathrm{DE}$

DOUTOR EM CIÊNCIAS

Programa: Matemática Aplicada

Orientador: Prof. Dr. Rodrigo Bissacot.

Durante o desenvolvimento deste trabalho o autor recebeu auxílio financeiro da CAPES/CNPq

São Paulo, Março 2019 



\section{Dedicatória}

A meus pais, Elmer e Nancy, e para meus avós Bernardo, Walter e Maria. 



\section{Agradecimentos}

Ao Instituto de matemática e estatística da Universidade de São Paulo, IME - USP, pelo seu acolhimento. Ao CAPES $\backslash \mathrm{CNPq}$, pelo seu apoio financeiro o qual foi de grande ajuda durante a minha permanência no Brasil.

Aos meus pais Elmer e Nancy pela educação que me deram. Aos meus irmãos Arnold, Karin e Shaarón pela sua confiança. A todos os meus tios por seu apoio, vocês sempre me ajudaram quando precisei, sempre serei grato a vocês. À minha avó Maria e aos meus avôs Bernardo e Walter por todo seu amor e carinho.

A meu orientador Prof. Dr. Rodrigo Bissacot pelo acompanhamento, disponibilidade, ensinamentos durante o desenvolvimento desta tese e por seus conselhos para minha formação como pesquisador. Sempre lembrarei dos seus ensinamentos em meus trabalhos futuros. Agradeço também sua amizade e a revisão da língua portuguesa. Desde já sou grato a ele. Ao Prof. Ricardo Freire pelo seu argumento da prova do item iii.) da Proposição 104.

A todos meus colegas do grupo de pesquisa do Prof. Rodrigo Bissacot por sua amizade e os conhecimentos compartilhados nos seminários do grupo. Em especial para Eric Endo por as muitas reuniões e discussões dos resultados desta tese, assim como, a ajuda ao escrever o artigo.

A todos meus amigos pelo seu apoio no momento certo, em especial para Cristian, Edu, Julio, Mariela e Reynaldo.

A todas as pessoas que não foram nomeadas mas fizeram parte da minha vida ao longo deste trabalho. 



\section{Resumo}

ELMER, R. BELTRAN. Medidas DLR e Transições de Fase Tipo Volume em shifts de Markov com Alfabeto Enumerável. 2019. 79 f. Tese Doutorado - Instituto de matemática e estatística, Universidade de São Paulo, São Paulo, 2019.

Introduzimos a extensão natural da definição de medida DLR para medidas sigma-finitas em shift de Markov com alfabeto enumerável. Provamos que o conjunto de medidas DLR contém o conjunto de medidas conformes associadas aos potenciais satisfazendo a condição de Walters. No caso BIP ou quando o potencial normaliza o operador de Ruelle, provamos que as noções de DLR e conformes coincidem. No shift de renewal obtemos uma caracterização de quando as medidas conformes são infinitas, estudamos o problema para descrever os casos em que o conjunto de medidas conformes pula de medidas finitas para infinitas quando consideramos altas e baixas temperaturas, respectivamente.

Palavras-chaves: medidas conformes, medidas DLR $\sigma$-finita, shift de Markov, transições de fase tipo volume, shift de renewal. 



\section{Abstract}

ELMER, R. BELTRAN. Infinite DLR Measures and Volume-Type Phase Transitions on Countable Markov Shifts. 2019. 79 p. PhD Thesis - Mathematics and Statistics Institute, São Paulo University, São Paulo, 2019.

We introduce the natural extension of the definition of DLR measure for sigma-finite measures on countable Markov shifts. We prove that the set of DLR measures contains the set of conformal measures associated to Walters potentials. In the BIP case or when the potential normalizes the Ruelle's operator we prove that the notions of DLR and conformal coincide. On renewal type shifts we obtained a characterization when the conformal measures are infinite, we study the problem to describe the cases when the set of conformal measures jumps from finite to infinite measures when we consider high and low temperatures, respectively.

Keywords: conformal measure, DLR measure $\sigma$-finite, Markov shift, phase transition type volume, shift of renewal. 



\section{Conteúdo}

1 Formalismo Termodinâmico em Shifts de Markov sobre Alfabetos Enumeráveis 5

1.1 Shift de Markov . . . . . . . . . . . . . . . . . . . . . . 5

1.2 Regularidade do Potencial . . . . . . . . . . . . . . . . . . . 8

1.3 Medidas Conformes . . . . . . . . . . . . . . . . . . . . . 9

1.3.1 Medidas Conformes no shift de Markov . . . . . . . . . . . . . . . . 10

1.4 Operador de Ruelle . . . . . . . . . . . . . . . . . . . . . 12

1.4.1 Operador de Transferência . . . . . . . . . . . . . . . . . . . . 12

1.4.2 Pressão de Gurevich ． . . . . . . . . . . . . . . . . . . . . . . 19

1.4.3 Teorema de Ruelle-Perron-Frobenius Generalizado . . . . . . . . . . . . . . 21

1.5 Medidas de Equilíbrio . . . . . . . . . . . . . . . . . . . 29

2 Medidas DLR $\quad 33$

2.1 Medidas DLR finitas . . . . . . . . . . . . . . . . . . . . . 33

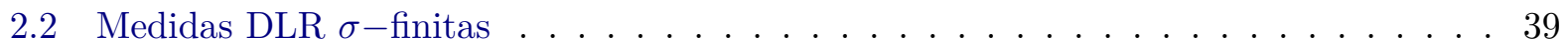

2.2.1 Esperança Condicional para Medidas $\sigma$-finitas . . . . . . . . . . . . . 39

2.2.2 Medidas DLR e Medidas Conformes no caso $\sigma$-finito . . . . . . . . . . 43

2.3 Equivalência entre Medidas DLR e de Equilíbrio . . . . . . . . . . . . . 57

3 Transições de Fase Tipo Volume em Shifts de Markov $\quad 65$

3.1 Existência de Transições de fase Tipo Volume . . . . . . . . . . . . . . . . . . 69

$\begin{array}{ll}\text { Conclusões e Questões Futuras } & 73\end{array}$

$\begin{array}{ll}\text { Referências } & 75\end{array}$

$\begin{array}{ll}\text { Índice Remissivo } & 79\end{array}$ 



\section{Lista de Notações}

$\mathcal{B}$

$\mathbb{N}$

$x_{m}^{n}$

$x_{m}^{\infty}$

$S$

$\sigma$

$\mathcal{M}\left(\Sigma_{A}\right) \quad$ Conjunto de medidas $\sigma$-finitas

$\mathcal{M}^{1}\left(\Sigma_{A}\right) \quad$ Conjunto de medidas de probabilidade

$\mathcal{M}_{\sigma}^{1}\left(\Sigma_{A}\right)$ Conjunto de medidas de probabilidade $\sigma$-invariante

$\mathcal{W}_{n} \quad$ Conjunto de palavras de tamanho $n$

$\mathcal{W}_{n}(c) \quad$ Conjunto de palavras de tamanho $n$ com simbolo final $c$

$\mathcal{W} \quad$ Conjunto de palavras finitas

$(\underline{a})_{p e r} \quad$ configuração periódica

$\mathbb{1}_{E} \quad$ Função característica do Boreliano $E$

$C_{b}\left(\Sigma_{A}\right) \quad$ Funções reais contínuas e acotadas definidas no shift de Markov 
$C_{c}\left(\Sigma_{A}\right) \quad$ Funções reais contínuas com suporte compacto definidas no shift de Markov

$L_{\phi} \quad$ Operador de Ruelle

$\hat{T} \quad$ Operador de Transferência

$P_{G}(\phi) \quad$ Pressão de Gurevich

q.t.p. $\quad$ Quase em todas partes

$\Sigma_{A} \quad$ Shift de Markov

$\underline{a} \quad$ Uma palavra

[a] $\quad$ Um cilindro 


\section{Introdução}

O formalismo termodinâmico, ou seja, o formalismo da física estatística de equilíbrio, originouse no trabalho de Boltzman e Gibbs e, posteriormente, foi adaptado à teoria dos sistemas dinâmicos nas obras clássicas do Sinai [Si], Ruelle [Ru04], [Ru67] e Bowen [Bo75]. D. Ruelle denominou este estudo por formalismo termodinâmico. A palavra "formalismo"é apropriada, uma vez que são obtidos teoremas abstratos e não apenas resultados para modelos específicos.

Em 1967 - 1968 Dobrushin [Dob], Landford [LaRu] e Ruelle [Ru67] introduziram o que hoje são conhecidas como medidas DLR. Estas medidas caracterizam as medidas de Gibbs (também chamada de distribuições Gibbsianas) em termos de uma família de probabilidades condicionais.

Nesta tese consideraremos shifts de Markov com alfabeto infinito enumerável. É conhecido que neste caso o shift de Markov em geral não é compacto, e estudaremos dois problemas. O primeiro é a equivalência entre a noção de medida DLR e medida conforme quando a medida for $\sigma$-finita. Para isso suporemos alguma regularidade tanto no shift de Markov como no potencial. O segundo problema é demonstrar que existe uma transição de fase tipo volume para a família de medidas conformes $\left\{\nu_{\beta \phi}\right\}_{\beta>0}$, onde cada medida $\nu_{\beta \phi}$ está associada ao potencial $\beta \phi$ com autovalor $e^{P_{G}(\beta \phi)}$, definidas em um shift renewal.

Quando o shift de Markov é topologicamente mixing e tem alfabeto finito, O. Sarig, em [Sa5], mostrou que para um potencial $\phi: \Sigma_{A} \rightarrow \mathbb{R}$ satisfazendo a condição de Walters existe uma única medida DLR e esta é $\lambda$-conforme, onde $\lambda=e^{P_{G}(\phi)}$. Uma prova diferente é dada por L. Cioletti e A. O. Lopes em [CiLo] no caso do full shift. Nosso primeiro teorema generaliza o resultado de O. Sarig para o caso de alfabeto infinito enumerável. Para isso consideramos a classe de shifts de Markov que possuem a propriedade $B I P^{1}$.

Teorema A. Sejam $\Sigma_{A}$ um shift de Markov topologicamente mixing com a propriedade BIP $e$ $\phi: \Sigma_{A} \rightarrow \mathbb{R}$ um potencial Walters com $\operatorname{Var}_{1} \phi<\infty$ e $P_{G}(\phi)<\infty$. Então existe uma única medida de probabilidade DLR e esta é $e^{P_{G}(\phi)}$-conforme.

Note que o teorema acima dá hipóteses no shift de Markov com alfabeto infinito enumerável para a existência de uma única medida DLR. Nas hipóteses do Teorema A, temos que o potencial é positivamente recorrente e a medida conforme associada a $\phi$, que denotaremos por $\nu$, é finita. Isto é consequência da propriedade $B I P$, veja [Sa4]. A demonstração deste teorema é feita em dois passos. Suponhamos que existe uma medida de probabilidade DLR $\mu$, primeiro demonstraremos que $\mu \ll \nu$. Logo existe uma função mensurável $\varphi: \Sigma_{A} \rightarrow[0, \infty)$ tal que $\varphi=\frac{\mathrm{d} \mu}{\mathrm{d} \nu}$. O segundo passo é usar a caracterização de medidas DLR dada por O. Sarig, Proposição 2.1 em [Sa5], para mostrar que $\varphi=1, \nu$-q.t.p.. Portanto $\mu=\nu$, para os detalhes da prova veja a demonstração do Teorema 93.

S. Muir em [Mu1] prova relações entre as medidas de equilíbrio e DLR para o espaço $\mathbb{N}^{d}$. Essas relações já haviam sido estudadas para o caso $S^{\mathbb{Z}^{d}}$, quando $|S|<\infty$, por [Kel] e [Ki] entre outros. Para obter resultados nessa direção foi provado o Teorema $B$, para isso primeiro provamos a seguinte proposição que é útil para a prova do teorema mencionado.

\footnotetext{
${ }^{1}$ Veja o item iii.) da Definição 2.
} 
Proposição A. Sejam $\Sigma_{A}$ um shift topologicamente mixing e $\phi: \Sigma_{A} \rightarrow \mathbb{R}$ um potencial recorrente que satisfaz a condição de Walters com $P_{G}(\phi)<\infty$. Consideremos $m:=h \mathrm{~d} \nu$ onde $h$ e $\nu$ é a autofunção e automedida do operador de Ruelle respectivamente, logo as seguintes afirmações são equivalentes:

i.) $m$ é $\phi-\mathrm{DLR}$;

ii.) $h(x)=\alpha, \nu$ - q.t.p., para algum $\alpha>0$.

Teorema B. Sejam $\Sigma_{A}$ um shift de Markov topologicamente mixing com a propriedade BIP $e$ $\phi: \Sigma_{A} \rightarrow \mathbb{R}$ um potencial que satisfaz a condição de Walters com $\operatorname{Var}_{1} \phi<\infty$ e $P_{G}(\phi)<\infty$. Então:

i.) $\{$ medida $\phi-\operatorname{DLR} \sigma-$ inavariante $\} \cap\left\{h_{(\cdot)}(\sigma)<\infty\right\} \subset\{$ medida $\phi-E q u i l i b r i o\}$;

ii.) $\{$ medida $\phi-$ Equilíbrio $\} \subset\{$ medidas $\widetilde{\phi}-\mathrm{DLR} \sigma-$ invariante $\}$,

onde $\widetilde{\phi}=\phi+\log h-\log h \circ \sigma-P_{G}(\phi)$.

A prova do teorema acima segue um roteiro análogo ao do Teorema $A$.

É conhecido que quando um shift de Markov topologicamente mixing tem alfabeto finito e o potencial satisfaz a condição de Walters, as medidas conformes são finitas. Este resultado é conhecido como o Teorema de Ruelle-Perron-Frobenius e foi demonstrado por D. Ruelle em [Ru68]. Quando o alfabeto é enumerável com cardinalidade infinita, O. Sarig estende esse teorema e o resultado é chamado de Teorema Generalizado de Ruelle-Perron-Frobenius, Teorema 3.4 em [Sa5]. Nesse caso, as medidas conformes não são necessariamente de probabilidade, estas são $\sigma$-finitas podendo dar massa infinita ao shift de Markov; veja o Exemplo 37 quando $\beta=1$.

O fato de existirem medidas conformes $\sigma$-finitas inspira estender a noção de medidas DLR, que foram introduzidas como medidas de probabilidade, ao caso $\sigma$-finito e também o estudo das relações destas com as medidas conformes. É assim que nesta tese estendemos a definição de medida DLR para o caso de medidas $\sigma$-finitas; veja a Definição 77. Note que o Teorema A mostra a equivalência entre medidas DLR e conformes para o caso de medidas com massa finita. Estamos interessados em mostrar as relações entre medidas DLR e conformes para o caso $\sigma$-finito com massa infinita; isso sera abordado nos Teoremas C e D.

Uma medida de probabilidade DLR é dada em termos de uma família de esperanças condicionais pré-definidas. Notemos que a esperança condicional está bem definida para este tipo de medida. Quando a medida é $\sigma$-finita, a esperança condicional pode ser definida sempre que a medida restrita à sub- $\sigma$-álgebra seja $\sigma$-finita com respeito a essa. A este fato chamaremos que a sub- $\sigma$-álgebra é compatível com a esperança condicional; veja a Definição 70. Para definir as medidas DLR no caso $\sigma$-finito, cada esperança condicional de $\left\{\mathbb{E}\left[\cdot \mid \sigma^{-n} \mathcal{B}\right]\right\}_{n \geq 1}$ deve estar bem definida, isto é equivalente a exigir que cada sub- $\sigma$-álgebra

$$
\sigma^{-1} \mathcal{B} \supset \sigma^{-2} \mathcal{B} \supset \ldots \supset \sigma^{-n} \mathcal{B} \supset \ldots
$$

seja compatível com a esperança condicional.

Nosso segundo resultado mostra que, impondo uma hipótese no potencial, cada esperança condicional de $\left\{\mathbb{E}\left[\cdot \mid \sigma^{-n} \mathcal{B}\right]\right\}_{n \geq 1}$ está bem definida em relação às medidas conformes. Assim, estas podem ser nossas medidas DLR no caso $\sigma$-finito.

Proposição B. Sejam $\Sigma_{A}$ o shift de Markov, $\phi: \Sigma_{A} \rightarrow \mathbb{R}$ um potencial mensurável e $\nu$ uma medida $(\phi, \lambda)$-conforme, para algum $\lambda>0$, tal que $\nu([a])<\infty$ para cada $a \in S$. Se $\left\|L_{\phi} \mathbb{1}\right\|_{\infty}<\infty$ então $\nu\left(\pi_{n}^{-1}\{a\}\right)<\infty$ para cada $n \in \mathbb{N}$ e $a \in S$. Em particular $\sigma^{-n} \mathcal{B}$ é compatível com a esperança condicional para cada $n \geq 1$. 
Notemos que a condição $\left\|L_{\phi} \mathbb{1}\right\|_{\infty}<\infty$ não implica que a medida conforme seja finita; veja o Exemplo 37. A ideia da prova é usar a forma integral da medida conforme; para mais detalhes veja a demonstração da Proposição 78.

Para um shift de Markov, qualquer medida conforme de massa finita associada a um potencial mensurável é DLR. Este fato foi demonstrado por O. Sarig; veja a Proposição 2.2 em [Sa5]. Nosso próximo resultado estende essa afirmação para medidas conformes $\sigma$-finitas.

Teorema C. Sejam $\Sigma_{A}$ o shift de Markov, $\phi: \Sigma_{A} \rightarrow \mathbb{R}$ um potencial mensurável e $\nu$ uma medida tal que $\nu\left(\pi_{n}^{-1}\{a\}\right)<\infty$ para cada $n \geq 0$ e a $\in$ S. Se $\nu$ é uma medida não-singular $e$ $(\phi, \lambda)$-conforme, para algum $\lambda>0$, então é $\phi-\mathrm{DLR}$.

Note que a hipótese $\nu\left(\pi_{n}^{-1}\{a\}\right)<\infty$, para cada $n \geq 0$ e $a \in S$ no teorema acima é para garantir que cada $\sigma^{-n} \mathcal{B}$ seja compatível com a esperança condicional, para cada $n \geq 1$. Assim cada esperança condicional de $\left\{\mathbb{E}\left[\cdot \mid \sigma^{-n} \mathcal{B}\right]\right\}_{n>1}$ está bem definida em relação à medida conforme $\nu$. Quando $\nu$ é finita, essa hipótese é satisfeita trivialmente. O roteiro da prova é o mesmo usado por O. Sarig para demonstrar o caso finito, porém a demonstração passa por duas passagens importantes. A primeira é estender o resultado de convergência de Martingais para medidas $\sigma$-finitas, veja o Teorema 76 , e a segunda é estender uma caracterização de medidas DLR dada por O. Sarig para o caso $\sigma$-finito, veja a Proposição 81. Para os detalhes da prova veja a demonstração do Corolário 85.

O próximo teorema é nosso último resultado sobre medidas DLR e conformes, este dá hipóteses necessárias e suficientes para que uma medida DLR no caso $\sigma$-finito seja conforme.

Teorema D. Sejam $\Sigma_{A}$ um shift de Markov, $\phi: \Sigma_{A} \rightarrow \mathbb{R}$ um potencial mensurável e $m$ uma medida $\phi$-DLR $\sigma$-invariante. Então $L_{\phi}(1)=\lambda m$-q.t.p. se, e somente, se $m$ é $(\phi, \lambda)$-conforme, onde $\lambda>0$.

O segundo problema abordado nesta tese é a transição de fase tipo volume em shifts renewal. Shifts renewal são estudados por G. Iommi e O. Sarig em [Io] e [Sa3], respectivamente. Nestes trabalhos são obtidos resultados que mostram um bom comportamento destes shifts em relação aos modos de recorrência e também estudam a existência de medidas maximizantes. Consideremos um potencial $\phi: \Sigma_{A} \rightarrow \mathbb{R}$ localmente Hölder tal que $\sup \phi<\infty$. O. Sarig mostrou que a família de potenciais $\{\beta \phi\}_{\beta>0}$ tem uma transição de fase tipo modo de recorrência, isto é, existe um único $\beta_{c} \in(0, \infty]$ tais que $\beta \phi$ é positivamente recorrente para cada $\beta<\beta_{c}$ e transiente para $\beta>\beta_{c}$, veja o Teorema $5 \mathrm{em} \mathrm{[Sa3].} \mathrm{Por} \mathrm{outro} \mathrm{lado} \mathrm{G.} \mathrm{Iommi} \mathrm{mostrou} \mathrm{que} \mathrm{quando} \beta_{c}=\infty$, neste caso existe uma medida que maximizante, isto é, existe $m \in \mathcal{M}_{\sigma}^{1}\left(\Sigma_{A}\right)$ tal que

$$
\int \phi \mathrm{d} m=\sup _{\nu \in \mathcal{M}_{\sigma}^{1}\left(\Sigma_{A}\right)}\left\{\int \phi \mathrm{d} \nu\right\}
$$

e quando $\beta_{c}<\infty$ não existe medida maximizante, para mais detalhes veja o Teorema $1.1 \mathrm{em}$ [Io].

O exemplo que deu origem ao estudo de transição de fase tipo volume em shifts renewal, foi o seguinte:

Exemplo A. Seja $\Sigma_{A}$ o shift renewal e $\phi: \Sigma_{A} \rightarrow \mathbb{R}$ o potencial dado por $\phi(x)=x_{0}-x_{1}$. Para cada $\beta>0$ pode-se mostrar que o potencial $\beta \phi$ é positivamente recorrente, neste caso $\beta_{c}=\infty$, e temos associada uma automedida $\nu_{\beta}$ tal que é finita para $\beta \in(0, \log 2)$ e infinita para cada $\beta \in[\log 2,+\infty)$, para mais detalhes veja o Exemplo 37 .

Como pode-se ver, existe um $\tilde{\beta}_{c}=\log 2 \leq \beta_{c}$ onde a medida muda de finita para infinita. A pergunta natural é se este fenômeno sempre acontece no shift renewal. Nosso próximo resultado dá uma resposta afirmativa.

Teorema E. Sejam $\Sigma_{A}$ um shift renewal e $\phi: \Sigma_{A} \rightarrow \mathbb{R}$ um potencial localmente Hölder tal que $\sup \phi<\infty$. Então existe $\tilde{\beta}_{c} \in\left(0, \beta_{c}\right]$ tal que $\nu_{\beta}$ é uma medida finita para $\beta \in\left(0, \tilde{\beta}_{c}\right)$ e é infinita para $\beta \in\left(\tilde{\beta}_{c}, \beta_{c}\right)$, onde $\nu_{\beta}$ é a automedida associada ao potencial $\beta \phi$. 
O teorema acima além de demonstrar uma transição de fase tipo volume, mostra que sempre existe medida finita para valores de $\beta>0$ pequenos. A prova é dada em dois passos. O primeiro passo é usar a forma integral das medidas conformes para cada potencial $\beta \phi, \beta<\beta_{c}$, e o comportamento dos shifts renewal. O segundo passo é estudar as propriedades da função $\psi:\left(0, \beta_{c}\right) \rightarrow \mathbb{R}$ definida por

$$
\psi(\beta)=\frac{P_{G}(\beta \phi)}{\beta} .
$$

Mostraremos que $\psi$ tem as seguintes propriedades:

- é contínua;

- é decrescente;

- $\lim _{\beta \rightarrow 0^{+}} \psi(\beta)=+\infty$.

Para os detalhes da prova, veja a demonstração do Teorema 105.

A tese está organizada da seguinte forma:

No Capítulo 1 fazemos uma introdução ao formalismo termodinâmico para shifts de Markov com alfabeto infinito enumerável abordando os principais resultados da área. Para isso, seguimos principalmente os trabalhos feitos por O. Sarig, [Sa1, Sa2, Sa4, Sa5], pois ele é a referência principal da área. Além disso, também citamos alguns resultados obtidos por V. Cyr, Y. Daon e O. Shwartz em [Cyr1], [Da] e [Sw], respectivamente. Neste capítulo estudamos conceitos como tipos de shift de Markov, regularidades do potencial, medidas conformes, pressão de Gurevich e medidas de equilíbrio. Os principais resultados deste capítulo são o Teorema Generalizado de Ruelle-PerronFrobenius (Teorema 36) que caracteriza cada potencial de acordo seu modo de recorrência, e o Teorema 46, que mostra a existência de uma autofunção e automedida para potenciais transientes. O primeiro teorema é dado por O. Sarig e o segundo por V. Cyr e O. Shwartz.

No Capítulo 2 estudaremos medidas DLR em shifts de Markov com alfabeto infinito enumerável, onde consideraremos medidas $\sigma$-finitas. O primeiro resultado importante é o Teorema 93 que mostra a equivalência entre a medida DLR e conforme quando o shift de Markov tem a propriedade $B I P$; é assim que temos uma classe de shifts de Markov onde a noção de medida DLR e de medida conforme são equivalentes mas para o caso de medidas finitas, pois a propriedade $B I P$ e $P_{G}(\phi)<\infty$ imposta no shift de Markov implica que a medida conforme é finita. Na Seção 2.2 abordamos o estudo das medidas DLR $\sigma$-finitas. Na Subseção 2.2.1 estendemos a definição de Esperança Condicional e o Teorema da Convergência de Martingais para o caso $\sigma$-finito, isto é necessário para a próxima subseção. Na Subseção 2.2.2 estendemos a definição de medida DLR para o caso $\sigma$-finito; note que esta coincide com a definição clássica quando a medida é de probabilidade, veja a Definição 77. Além disso, mostraremos que, impondo uma condição no potencial, as medidas conformes $\sigma$-finitas são candidatas a ser DLR, veja a Proposição 78. Neste capítulo estendemos uma caracterização das medidas DLR para o caso $\sigma$-finito e demonstramos que toda medida conforme $\sigma$-finita é DLR, veja a Proposição 81 e o Corolário 85 respectivamente. Terminaremos este capítulo com o Teorema 89 que dá condições para uma medida DLR no shift de Markov ser conforme; a reciproca é falsa no caso geral.

No Capítulo 3 abordaremos o problema de transição de fase tipo volume para shifts renewal. Na primeira parte deste capítulo estudaremos dois resultados importantes sobre shifts renewal, os Teoremas 99 e 101 dados por O. Sarig em [Sa3], e G. Iommi em [Io], respectivamente. O resultado principal deste capítulo aparece na Seção 3.1; Teorema 105. Este mostra a existência de transição de fase tipo volume na família de automedidas $\nu_{\beta}$ correspondente aos potenciais $\beta \phi$ para $0<\beta<\beta_{c}$. Além disso, mostraremos que um shift de Markov com um comportamento análogo ao shift renewal não tem transição de fase tipo volume, veja a Proposição 107. 


\section{Capítulo 1}

\section{Formalismo Termodinâmico em Shifts de Markov sobre Alfabetos Enumeráveis}

\subsection{Shift de Markov}

Seja $S$ um conjunto enumerável com cardinalidade infinita, chamaremos $S$ de alfabeto no decorrer deste trabalho e podemos pensar como sendo $S=\mathbb{N}$. Seja $A: S \times S \rightarrow\{0,1\}$ uma matriz de zeros e uns sem colunas ou linhas que são todas zeros. O shift de Markov denotado por $\Sigma_{A}$ são as sequências permitidas (segundo a matriz $A$ ), isto é,

$$
\Sigma_{A}:=\left\{x=\left(x_{0}, x_{1}, \ldots, x_{i}, \ldots\right) \in S^{\mathbb{N}_{0}}: A\left(x_{i}, x_{i+1}\right)=1, \forall i \geq 0\right\} .
$$

Seja $w=\left(w_{0}, w_{1}, \ldots, w_{k-1}\right)$, com $w_{i} \in S$ para $0 \leq i \leq k-1$, definimos o cilindro de $w$ com comprimento $k \in \mathbb{N}$ pelo conjunto

$$
[w]:=\left\{x \in \Sigma_{A} \mid x_{i}=w_{i} \text { para } 0 \leq i \leq k-1\right\} .
$$

Uma palavra permitida ${ }^{1}$ de tamanho $n \in \mathbb{N}$ é uma concatenação finita $\underline{\omega}=\left(\omega_{0}, \omega_{1}, \ldots, \omega_{n-1}\right)$ de $n$ símbolos em $S$ tal que $[\underline{\omega}] \neq \emptyset$. Para cada $n \in \mathbb{N}$, denotaremos por $\mathcal{W}_{n}$ o conjunto de palavras com tamanho $n$.

Fixado $0<\lambda<1$, define-se a métrica em $\Sigma_{A}$ por

$$
d_{\lambda}(x, y)=\lambda^{\inf \left\{j: x_{j} \neq y_{j}\right\}} .
$$

Note que cada cilindro é aberto e fechado em relação a métrica $d_{\lambda}$ e a topologia gerada pela métrica tem os cilindros como base.

O shift de Markov $\left(\Sigma_{A}, d_{\lambda}\right)$ é um espaço métrico não-compacto e separável. Quando o alfabeto é finito sabe-se que o espaço é compacto. A seguir temos uma condição na matriz de transição para que cada cilindro seja compacto.

Proposição 1. Seja $\Sigma_{A}$ um shift de Markov. Então $\sum_{b \in S} A(a, b)<\infty$ para cada a $\in$ Se, e somente, se cada cilindro é compacto.

Demonstração. Consideremos $\sum_{b \in S} A(a, b)<\infty$ para todo $a \in S$. Para cada $a \in S$ e $n \in \mathbb{N}$

\footnotetext{
${ }^{1}$ Ao longo deste trabalho chamaremos simplesmente de palavra
} 
definamos

$$
A^{n}(a):=\left\{j_{1}, j_{2}, \ldots j_{n} \in S: A\left(a, j_{1}\right) A\left(j_{1}, j_{2}\right) \ldots A\left(j_{n-1}, j_{n}\right)=1\right\},
$$

por hipótese temos que $|A(a)|<\infty$.

Seja $[\underline{\omega}]:=\left[\omega_{0}, \omega_{1}, \ldots, \omega_{m-1}\right]$ um cilindro arbitrário de tamanho $m \in \mathbb{N}$. Desde que

$$
[\underline{\omega}]=\left\{\omega_{0}\right\} \times\left\{\omega_{1}\right\} \times \ldots \times\left\{\omega_{m-1}\right\} \times A\left(\omega_{m-1}\right) \times A^{2}\left(\omega_{m-1}\right) \times \ldots A^{n}\left(\omega_{m-1}\right) \times \ldots
$$

e $A^{n}\left(\omega_{m-1}\right)<\infty$ para cada $n \in \mathbb{N}$ temos que $[\underline{\omega}]$ é compacto na topologia produto. Como a topologia produto coincide com a topologia da métrica temos que $[\underline{\omega}]$ é compacto.

Suponhamos que $[\underline{\omega}]:=\left[\omega_{0}, \omega_{1}, \ldots, \omega_{m-1}\right]$ seja um cilindro compacto de tamanho $m \in \mathbb{N}$ e $\sum_{b \in S} A\left(\omega_{m-1}, b\right)=\infty$. Logo $[\underline{\omega}]$ pode se escrever como

$$
[\underline{\omega}]=\bigcup_{b \in S}[\underline{\omega} b],
$$

onde $\{[\underline{\omega} b]\}_{A\left(\omega_{m-1}, b\right)}$ é uma cobertura de $[\underline{\omega}]$, isso contradiz o fato que $[\underline{\omega}]$ é compacto.

Seja $\sigma$ a dinâmica em $\Sigma_{A}$ definida por

$$
\begin{aligned}
\sigma: \Sigma_{A} & \longrightarrow \Sigma_{A} \\
x=\left(x_{0}, x_{1}, \ldots\right) & \longmapsto \sigma(x)=\left(x_{1}, x_{2}, \ldots\right),
\end{aligned}
$$

esta aplicação é chamada de aplicação shift.

A seguir vamos caracterizar os diferentes tipos de shifts de Markov que aparecem na literatura do formalismo termodinâmico.

Definição 2. Dizemos que um shift de Markov $\Sigma_{A}$ é:

i.) Transitivo, quando para todo $a, b \in S$ existe $N>0$, tal que $[a] \cap \sigma^{-N}[b] \neq \emptyset$.

iii.) Topologicamente mixing, quando para todo $a, b \in S$ existe $N_{a, b}>0$, tal que para todo $n>N_{a, b}$, temos $[a] \cap \sigma^{-n}[b] \neq \emptyset$.

iii.) Satisfaz a propriedade $B I P$ se, e somente, se existe um conjunto finito $B=\left\{b_{1}, b_{2}, \ldots, b_{m}\right\}$ de elementos em $S$ tal que para qualquer $a \in S$ existem $i, j \in\{1,2, \ldots, m\}$ tais que $A\left(b_{i}, a\right)=$ $A\left(a, b_{j}\right)=1$.

iv.) Primitivo, quando existe $k_{0} \in \mathbb{N}$ e um sub-alfabeto $\mathbb{F} \subset S$ tal que qualquer par de símbolos do alfabeto $S$ pode ser conectado por uma palavra com exatamente $k_{0}$ símbolos em $\mathbb{F}$. Dizemos que $\Sigma_{A}$ é finitamente primitivo quando $\mathbb{F}$ é finito.

É fácil ver que todo shift de Markov topologicamente mixing é transitivo no entanto a recíproca não sempre é verdade. O seguinte lema mostra que as condições finitamente primitivo e BIP são equivalentes em shifts de Markov topologicamente mixing. A prova pode ser encontrada em [Sa5].

Lema 3. Seja $\Sigma_{A}$ um shift topologicamente mixing, então $\Sigma_{A}$ tem a propriedade BIP se, e somente, se $\Sigma_{A}$ é finitamente primitivo.

Vale ressaltar que no lema anterior não precisamos o fato do shift ser topologicamente mixing para mostrar que todo shift finitamente primitivo satisfaz a propriedade $B I P$.

Proposição 4. Seja $\Sigma_{A}$ um shift de Markov transitivo. Logo $\sum_{b \in S} A(a, b)<\infty$ para cada $a \in S$ se, e somente, se $\Sigma_{A}$ é localmente compacto. 
Demonstração. A primeira implicação é uma consequência direta da Proposição 1. Provemos a recíproca, suponhamos que existe $s \in S$ tal que $\sum_{b \in S} A(s, b)=\infty$. Seja $x_{0} \in S$ e $\underline{\omega}, \underline{v}$ duas palavras tais que $x=\left(x_{0}, \underline{\omega}, \underline{s} \underline{\underline{v}}\right)_{\text {per }} \in \Sigma_{A}$, como $\Sigma_{A}$ é um espaço Hausdorff, localmente compacto temos que existe um aberto $U \ni x$ tal que $\bar{U}$ é compacto.

Desde que $U$ é aberto e a família de cilindros é a base da topologia temos que existe $n \in \mathbb{N}$ e um cilindro $[\underline{\theta}]$ de tamanho $n$ tal que

$$
x \in[\underline{\theta}] \subset \bar{U} .
$$

Como $[\underline{\theta}]$ é fechado temos que é compacto, assim todo subconjunto fechado tem que ser compacto mas $\left[x_{0}, \underline{\omega}, s\right] \subset[\underline{\theta}]$ não é compacto. Portanto $\sum_{b \in S} A(a, b)<\infty$ para todo $a \in S$.

A seguir apresentamos um shift topologicamente mixing que não satisfaz a propriedade $B I P$ e será estudado no Capítulo 3.

Definição 5 (shift renewal). Consideremos a matriz de transição $\left(A_{i, j}\right)_{\mathbb{N} \times \mathbb{N}}$ com entradas $A(1, i)$, $A(i, i-1)$ igual a 1 , para cada $i \geq 2, A(1,1)=1$ e o resto das entradas igual a zero. $O$ shift de Markov dada pela matriz $A$ é chamado de shift renewal. O elemento $1 \in \mathbb{N}$ é chamado de vértice de renewal.

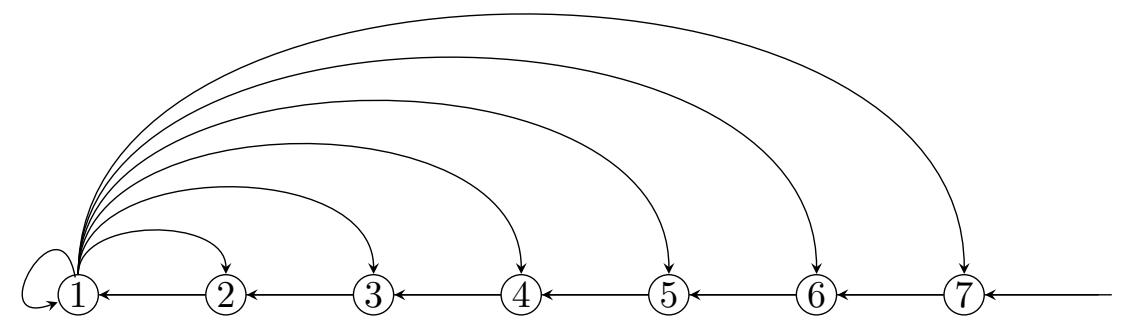

Figura 1.1: shift renewal

Note que o shift renewal, representado pelo grafo da Figura 1.1, é topologicamente mixing e não satisfaz a propriedade $B I P$.

Observação 6. G. Iommi em [Io], inspirado no shift renewal define uma classe de shifts de Markov que tem o comportamento do shift renewal e chama de classe renewal. A classe renewal e denotada por $\Sigma_{\mathcal{R}}$ e são todos os shifts topologicamente mixing tais que para cada $n \in \mathbb{N}$ há no máximo uma palavra admissível $x_{0} x_{1} \ldots x_{n-1}$ de tamanho $n$ satisfazendo $A\left(x_{n-1}, 1\right)=1$ e $x_{i}=1$ se, e somente, se $i=0$.

Exemplo 7. Consideremos $\left\{d_{n}\right\}_{n \in \mathbb{N}}$ uma sequência crescente em $\mathbb{N}$ tal que $\lim _{n \rightarrow \infty} d_{n}=+\infty$. Notemos que o shift representado pelo grafo da Figura 1.2 é um shift que pertence a classe $\Sigma_{\mathcal{R}} e$ denotaremos por $\Sigma_{\mathcal{R}_{1}}$.

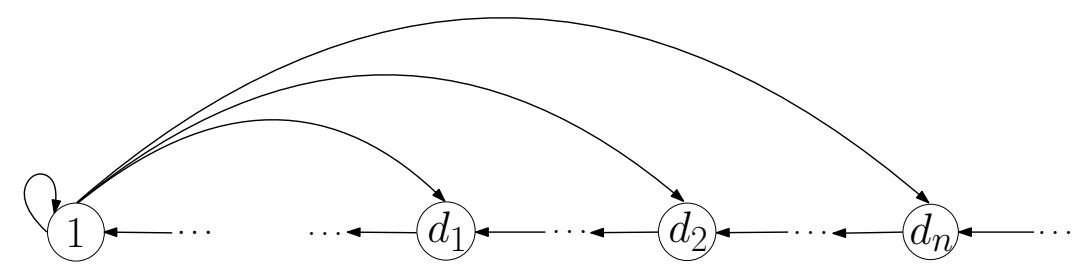

Figura 1.2 


\subsection{Regularidade do Potencial}

Uma função real e contínua definida em $\Sigma_{A}$ será chamada de potencial. Dado qualquer potencial $\phi: \Sigma_{A} \rightarrow \mathbb{R}$, para cada $n \in \mathbb{N}$ definimos:

i.) a $n$-ésima soma ergódica de $\phi$ por

$$
\phi_{n}:=\sum_{i=0}^{n-1} \phi \circ \sigma^{i}
$$

ii.) a n-ésima variação de $\phi$ como

$$
\operatorname{Var}_{n} \phi:=\sup \left\{|\phi(x)-\phi(y)|: x_{0}=y_{0}, x_{1}=y_{1}, \ldots, x_{n-1}=y_{n-1}\right\} \text {. }
$$

A seguir definimos algumas regularidades no potencial.

Definição 8. Dado um potencial $\phi: \Sigma_{A} \rightarrow \mathbb{R}$, dizemos que $\phi$ :

i.) é somável se

$$
\sum_{i \geq 1} e^{\left.\sup \phi\right|_{[i]}}<\infty
$$

onde $\left.\sup \phi\right|_{[i]}:=\sup _{x \in[i]} \phi(x)$.

ii.) é localmente Hölder quando existem uma constante $H_{\phi}>0$ e $r \in(0,1)$ tais que, para todo inteiro $k \geq 2$, temos

$$
\operatorname{Var}_{k} \phi \leq H_{\phi} r^{k}
$$

iii.) tem variação somável se

$$
\operatorname{Var} \phi:=\sum_{k \geq 2} \operatorname{Var}_{k} \phi<\infty
$$

iv.) satisfaz a condição de Walters, se

$$
\sup _{n \geq 1} \operatorname{Var}_{n+k} \phi_{n}<\infty, \text { para cada } k \geq 1, e \lim _{k \rightarrow \infty} \sup _{n \geq 1} \operatorname{Var}_{n+k} \phi_{n}=0 .
$$

A condição localmente Hölder significa que a $k$-ésima variação, $\operatorname{Var}_{k} \phi$, decai exponencialmente a zero quando $k \rightarrow \infty$. Claramente a condição localmente Hölder é mais forte que a variação somável. Cada uma destas condições implicam que o potencial é uniformemente contínuo, no entanto elas não implicam que o potencial seja limitado, pois $\sup _{x, y \in \Sigma_{A}}|\phi(x)-\phi(y)|<\infty$ não está incluída nestas definições.

A seguinte proposição mostra que a condição de Walters é mais fraca que a variação somável. A prova pode ser encontrada em [Sa5].

Proposição 9. Sejam $\Sigma_{A}$ um shift de Markov e $\phi: \Sigma_{A} \rightarrow \mathbb{R}$ um potencial com variação somável. Para todo $n \geq 1$ e qualquer palavra $\underline{a}=\left(a_{0}, a_{1}, \ldots, a_{n-1}\right)$ de tamanho $n$, se $x, y \in \sigma\left[a_{n-1}\right]$ e $x_{0}=y_{0}, x_{1}=y_{1}, \ldots, x_{m-1}=y_{m-1}$, ent $\tilde{a} o$

$$
\left|\phi_{n}(\underline{a} x)-\phi_{n}(\underline{a} y)\right| \leq \sum_{k=m+1}^{\infty} \operatorname{Var}_{k} \phi .
$$

A condição de Walters foi introduzida por P. Walters em [Wal78] para shifts de Markov com alfabeto finito, isto é, shifts de Markov compactos. A definição original não incluía a condição $\sup _{n \geq 1} \operatorname{Var}_{n+k} \phi_{n}<\infty$ para $k \geq 1$ pois no caso compacto esta é uma consequência de 
$\lim _{k \rightarrow \infty} \sup _{n \geq 1} \operatorname{Var}_{n+k} \phi_{n}=0$. A classe de potenciais que satisfazem a condição de Walters é a maior classe de potenciais onde o formalismo termodinâmico está desenvolvido. A seguir mostramos um exemplo de potenciais que satisfazem a condição de Walters.

Exemplo 10. Seja $\phi: \Sigma_{A} \rightarrow \mathbb{R}$ um potencial tal que $\phi$ depende do primeiro símbolo, isto é, $\phi(x)=x_{0}$. É fácil ver que $\operatorname{Var}_{n+k}\left(\phi_{n}\right)=0$ para cada $n \in \mathbb{N}$ e cada $k \geq 1$, logo $\phi$ satisfaz a condição de Walters.

Lema 11. Sejam $\Sigma_{A}$ um shift de Markov e $\phi: \Sigma_{A} \rightarrow \mathbb{R}$ um potencial que satisfaz a condição de Walters com $\operatorname{Var}_{1} \phi<\infty$. Existe $M>0$ tal que para cada cilindro [ $\left.\underline{w}\right]$ de tamanho $n \in \mathbb{N}$ temos

$$
\left|\phi_{n}(x)-\phi_{n}(y)\right| \leq M
$$

onde $x, y \in[\underline{w}]$.

Demonstração. Definamos $M_{0}:=\sup _{n \geq 1} \operatorname{Var}_{n+1} \phi_{n}+\operatorname{Var}_{1} \phi$. Dado [ $\left.\underline{w}\right]$ um cilindro de tamanho $n \geq 1$, definamos

$$
\underline{\phi_{n}}[\underline{w}]:=\inf _{x \in[w]} \phi_{n}(x), \quad \text { e } \quad \overline{\phi_{n}}[\underline{w}]:=\sup _{x \in[w]} \phi_{n}(x) .
$$

Notemos que para cada $x \in[\underline{w}]$ temos

$$
\left|\underline{\phi_{n}}[\underline{w}]-\phi_{n}(x)\right| \leq M_{0}, \quad \text { e } \quad\left|\overline{\phi_{n}}[\underline{w}]-\phi_{n}(x)\right| \leq M_{0} .
$$

Das desigualdades de (1.2) obtemos a desigualdade (1.1).

Observação 12. Nas condições do lema acima temos que dado um cilindro $[\underline{w}]$ de tamanho $n \in \mathbb{N}$, existem constantes finitas $C_{1}(\underline{w})$ e $C_{2}(\underline{w})$ tal que

$$
C_{1}(\underline{w})<\phi_{n}(x)<C_{2}(\underline{w}), \quad \text { para todo } x \in[\underline{w}] .
$$

\subsection{Medidas Conformes}

M. Denker e M. Urbański introduzem uma definição geral de medida conforme, Definição 13, para espaços métricos compactos e estudam suas propriedades assim como as condições suficientes para sua existência, veja [DeUr]. As medidas conforme aparecem originalmente em [Pa], e são obtidas em conjuntos limite de subgrupos discretos finitamente gerados de aplicações conformes no espaço hiperbólico.

Definição 13 ([DeUr]). Sejam $(\Omega, F)$ um espaço mensurável, $T: \Omega \rightarrow \Omega$ um endomorfismo mensurável e $\phi: \Omega \rightarrow[0, \infty)$ um potencial mensurável. Uma medida $\mu$ em $\mathcal{F}$ é chamada $\phi$-conforme se,

$$
\mu(T(A))=\int_{A} \phi(x) \mathrm{d} \mu(x)
$$

onde $A \in \mathcal{F}$ é tal que $T(A)$ é mensurável e $T: A \rightarrow T(A)$ é invertível. O conjunto $A$ é chamado de especial.

Da Equação (1.3) temos que, estudar a existência de medidas conformes é equivalente a estudar condições para que $\mu \circ T \ll \mu$ e que a derivada de Radon-Nikodým seja $\frac{\mathrm{d} \mu \circ T}{\mathrm{~d} \mu}=\phi$ em cada conjunto especial. As medidas satisfazendo a Equação (1.3) desempenham um papel importante no estudo da teoria de equilíbrio em sistemas dinâmicos, para mais detalhes veja [DeUr]. 


\subsubsection{Medidas Conformes no shift de Markov}

Ao longo deste trabalho denotaremos por $\mathcal{B}$ a $\sigma$-álgebra de Borel em $\Sigma_{A}$.

Definição 14. Sejam $\Sigma_{A}$ um shift de Markov e $\nu$ uma medida em $\mathcal{B}$. Denotamos $\nu \odot \sigma$ a medida definida por

$$
\nu \odot \sigma(E):=\sum_{a \in S} \nu(\sigma(E \cap[a])), \quad E \in \mathcal{B} .
$$

Notemos que:

i) $\nu \odot \sigma$ está bem definida.

Para isso devemos mostrar que para cada $a \in S$ e cada $E \in \mathcal{B}, \sigma(E \cap[a]) \in \mathcal{B}$.

Com efeito, fixado $a \in S$ consideremos,

$$
\begin{aligned}
\left.\sigma\right|_{[a]}:[a] & \longrightarrow A=\left\{y \in \Sigma_{A} \mid a y \in \Sigma_{A}\right\} \\
x & \left.\longmapsto \sigma\right|_{[a]}(x)=\sigma(x)
\end{aligned}
$$

é fácil ver que $\left.\sigma\right|_{[a]}$ é bijetora e um homeomorfismo sobre sua imagem. Para cada $E \in \mathcal{B}$ temos que $E \cap[a]$ é mensurável em $\left.[a] \cap \mathcal{B} \operatorname{logo} \sigma\right|_{[a]}(E \cap[a])$ é mensurável em $A$ e por consequência é mensurável em $\mathcal{B}$, pois $A=\cup_{b \in S \mid A(a, b)=1}[b]$ é mensurável em $\mathcal{B}$.

Notemos que a afirmação foi consequência do fato que $\left.\sigma\right|_{[a]}:[a] \rightarrow \sigma[a]$ é um homeomorfismo, para cada $a \in S$.

ii) Em geral $(\nu \odot \sigma)(E) \neq \nu \circ \sigma(E)$.

É fácil ver que $\nu \odot \sigma([a c] \cup[b c])=2 \nu([c])$, no entanto $\nu(\sigma([a c] \cup[b c]))=\nu[c]$. Podemos entender $(\nu \odot \sigma)(E)$ como a medida de $\sigma(E)$ contando multiplicidades.

Definição 15. Sejam $(\Omega, \mathcal{F}, \mu)$ um espaço de medida e $T: \Omega \rightarrow \Omega$ uma aplicação mensurável. A medida $\mu$ (ou a aplicação $T$ ) é chamada não-singular, se $\mu \circ T^{-1} \sim \mu$, isto é, $\mu\left(T^{-1} E\right)=0$ se, e somente, se $\mu(E)=0, E \in \mathcal{F}$.

Lema 16. Sejam $\Sigma_{A}$ um shift de Markov e $\nu$ uma medida em $\mathcal{B}$. Então:

i.) Para toda função mensurável $f: \Sigma_{A} \rightarrow[0, \infty)$, temos

$$
\int_{\Sigma_{A}} f \mathrm{~d} \nu \odot \sigma=\sum_{a \in S} \int_{\sigma[a]} f(a x) \mathrm{d} \nu(x) .
$$

ii.) Se $\nu$ é não-singular então, $\nu \ll \nu \odot \sigma$.

Demonstração.

i) Seja $E \in \mathcal{B}$ e consideremos $f:=\mathbb{1}_{E}$. Logo usando a definição da medida $\nu \odot \sigma$ e o fato que $\left.\sigma\right|_{[a]}:[a] \rightarrow \sigma[a]$ é um homeomorfismo, temos

$$
\begin{aligned}
\int_{\Sigma_{A}} \mathbb{1}_{E}(x) \mathrm{d} \nu \odot \sigma(x) & =\int_{E} \mathrm{~d} \nu \odot \sigma \\
& =\nu \odot \sigma(E) \\
& =\sum_{a \in S} \nu(\sigma(E \cap[a])) \\
& =\sum_{a \in S} \int_{\sigma(E \cap[a])} \mathrm{d} \nu
\end{aligned}
$$




$$
\begin{aligned}
& =\sum_{a \in S} \int_{\Sigma_{A}} \mathbb{1}_{\left.\sigma\right|_{[a]}(E \cap[a])}(x) \mathrm{d} \nu(x) \\
& =\sum_{a \in S} \int_{\Sigma_{A}} \mathbb{1}_{E \cap[a]}\left(\left.\sigma\right|_{[a]} ^{-1} x\right) \mathrm{d} \nu(x) \\
& =\sum_{a \in S} \int_{\Sigma_{A}} \mathbb{1}_{E}\left(\left.\sigma\right|_{[a]} ^{-1} x\right) \mathbb{1}_{[a]}\left(\left.\sigma\right|_{[a]} ^{-1} x\right) \mathrm{d} \nu(x) \\
& =\sum_{a \in S} \int_{\Sigma_{A}} \mathbb{1}_{E}(a x) \mathbb{1}_{\sigma[a]}(x) \mathrm{d} \nu(x) \\
& =\sum_{a \in S} \int_{\sigma[a]} \mathbb{1}_{E}(a x) \mathrm{d} \nu(x) .
\end{aligned}
$$

As igualdades acima se estendem facilmente para funções simples. Seja $f \geq 0$ uma função mensurável, logo existe uma sequencia de funções simples $\left\{s_{n}\right\}_{n \geq 1}$ tais que $0 \leq s_{1} \leq s_{2} \leq \ldots \leq$ $s_{n} \leq \ldots \leq f$ e $s_{n}(x) \stackrel{n \rightarrow \infty}{\longrightarrow} f(x)$ para todo $x \in \Sigma_{A}$. Pelo Teorema da Convergência Monótona temos

$$
\begin{aligned}
\int_{\Sigma_{A}} f \mathrm{~d} \nu \odot \sigma & =\lim _{n \rightarrow \infty} \int_{\Sigma_{A}} s_{n} \mathrm{~d} \nu \odot \sigma \\
& =\lim _{n \rightarrow \infty} \sum_{a \in S} \int_{\sigma[a]} s_{n}(a x) \mathrm{d} \nu(x) \\
& =\sum_{a \in S} \lim _{n \rightarrow \infty} \int_{\sigma[a]} s_{n}(a x) \mathrm{d} \nu(x) \\
& =\sum_{a \in S} \int_{\sigma[a]} f(a x) \mathrm{d} \nu(x) .
\end{aligned}
$$

ii) Seja $E \in \mathcal{B}$ tal que $\nu \odot \sigma(E)=0$, isto é, $\nu(\sigma(E \cap[a]))=0$ para todo $a \in S$. Por hipótese $\nu$ é não-singular e então temos $\nu\left(\left.\sigma^{-1} \sigma\right|_{[a]}(E \cap[a])\right)=0$. Como $\left.\left.\left.\sigma\right|_{[a]} ^{-1} \sigma\right|_{[a]}(E \cap[a]) \subset \sigma^{-1} \sigma\right|_{[a]}(E \cap[a])$ e $\left.\sigma\right|_{[a]}$ é um homeomorfismo local temos $\nu(E \cap[a])=0$ para todo $a \in S$, $\operatorname{logo} \nu(E)=0$. Portanto $\nu \ll \nu \odot \sigma$.

Pelo lema anterior temos que a partir de uma medida $\mu$ não-singular em $\mathcal{B}$ a derivada de Radon-Nikodým $\frac{\mathrm{d} \mu}{\mathrm{d} \mu \odot \sigma}$ está bem definida.

Definição 17 ([Sa5]). Sejam $\Sigma_{A}$ um shift de Markov, $\phi: \Sigma_{A} \rightarrow \mathbb{R}$ um potencial mensurável e $\mu$ uma medida $\sigma$-finita e não-singular em $\mathcal{B}$. Dizemos que $\mu$ é $(\phi, \lambda)$-conforme, se

$$
\frac{\mathrm{d} \mu}{\mathrm{d} \mu \odot \sigma}=\lambda^{-1} e^{\phi}, \quad \mu-\text { q.t.p. },
$$

onde $\lambda>0$.

Na Proposição 19 mostraremos a relação entre as medidas conformes segundo O. Sarig e DenkerUrbanski, Definições 17 e 13 respectivamente, para isso primeiro mostraremos o seguinte lema.

Lema 18. Sejam $\Sigma_{A}$ um shift de Markov e $\mu$ uma medida em $\mathcal{B}$. A família dos cilindros $\{[a]:$ a $\in$ $S\}$ formam uma partição mensurável de elementos especiais para $\Sigma_{A}$.

Demonstração. Seja $a \in S$ arbitrário, temos que $\sigma:[a] \rightarrow \sigma[a]$ é bijetora e um homeomorfismo.

Proposição 19. Sejam $\Sigma_{A}$ um shift de Markov, $\mu$ uma medida em $\mathcal{B}$ e $\phi: \Sigma_{A} \rightarrow \mathbb{R}$ uma função mensurável. Então $\mu$ é e $e^{\phi}$-conforme segundo Denker-Urbanski (Definição 13) se, e somente, se $\mu$ é não-singular e $(-\phi, 1)$-conforme segundo Sarig (Definição 17). 
Demonstração. Consideremos $\mu$ uma medida $e^{\phi}$-conforme segundo Denker-Urbanski. Então

$$
\mu(\sigma(E \cap[a]))=\int_{E \cap[a]} e^{\phi} \mathrm{d} \mu,
$$

onde $E \in \mathcal{B}$ e $a \in S$.

Seja $E \in \mathcal{B}$, usando a Equação (1.5) temos

$$
\begin{aligned}
\mu \odot \sigma(E) & =\sum_{a \in S} \mu(\sigma(E \cap[a])) \\
& =\sum_{a \in S} \int_{E \cap[a]} e^{\phi} \mathrm{d} \mu \\
& =\int_{E} e^{\phi} \mathrm{d} \mu,
\end{aligned}
$$

$\log 0$

$$
\frac{\mathrm{d} \mu \odot \sigma}{\mathrm{d} \mu}=e^{\phi} .
$$

Portanto $\frac{\mathrm{d} \mu}{\mathrm{d} \mu \odot \sigma}=e^{-\phi}$. Além disso a Equação (1.6) implica que $\mu$ é não-singular. Portanto $\mu$ é uma medida $(-\phi, 1)-$ conforme segundo Sarig. A prova da recíproca é direta.

\subsection{Operador de Ruelle}

Consideremos $|S|<\infty$ e $\phi: \Sigma_{A} \rightarrow \mathbb{R}$ um potencial contínuo. D. Ruelle em [Ru76] definiu o operador $L_{\phi}: C\left(\Sigma_{A}\right) \rightarrow C\left(\Sigma_{A}\right)$ da seguinte maneira

$$
L_{\phi} f(x):=\sum_{\sigma y=x} e^{\phi(y)} f(y)
$$

onde $f: \Sigma_{A} \rightarrow \mathbb{R}$ é uma função contínua. Note que o operador $L_{\phi}$ está bem definido e é chamado de operador de Ruelle.

Notemos que em geral quando o alfabeto é enumerável com $|S|=\infty$ o somatório da Equação (1.7) pode explodir pois o conjunto das pre-imagens, $\left\{y \in \Sigma_{A} \mid \sigma y=x\right\}$, pode ser infinito e neste caso o operador de Ruelle não está bem definido. No entanto existem várias maneiras de definir o operador de Ruelle e para isso devemos considerar alguma regularidade no potencial ou no shift de Markov. A seguir estudaremos o operador de transferência o qual nos fornecerá as hipóteses necessárias para definir o operador de Ruelle, para mais detalhes veja [Aar] e [Sa5].

\subsubsection{Operador de Transferência}

Definição 20. Sejam $(\Omega, \mathcal{F}, \mu)$ um espaço de medida $\sigma$-finita, $\mu$ não-singular e $T: \Omega \rightarrow \Omega$ uma aplicação mensurável. O operador $\widehat{T}: L^{1}(\mu) \rightarrow L^{1}(\mu)$ definido por

$$
\widehat{T} f:=\frac{\mathrm{d} \mu_{f} \circ T^{-1}}{\mathrm{~d} \mu}, \quad \text { onde } \mathrm{d} \mu_{f}:=f \mathrm{~d} \mu,
$$

e é chamado de operador de transferência.

Notemos que o operador $\widehat{T}$ está bem definido como operador em $L^{1}(\mu)$ pois:

i.) A derivada de Radon-Nikodým está bem definida. 
Seja $E \in \mathcal{F}$ tal que $\mu(E)=0$, como $\mu$ é não-singular $\operatorname{logo} \mu\left(T^{-1} E\right)=0$. Assim para cada $f \in L^{1}(\mu)$ temos

$$
\mu_{f}\left(T^{-1} E\right)=\int_{T^{-1} E} f \mathrm{~d} \mu=0 .
$$

Logo $\mu_{f} \circ T^{-1} \ll \mu$.

ii.) Para toda $f \in L^{1}(\mu)$ temos $\widehat{T} f \in L^{1}(\mu)$.

Seja $f \in L^{1}(\mu)$, logo

$$
\begin{aligned}
\|\widehat{T} f\|_{1} & =\int \operatorname{sgn}(\widehat{T} f) \cdot \hat{T} f \mathrm{~d} \mu=\int \operatorname{sgn}(\widehat{T} f) \mathrm{d} \mu_{f} \circ T^{-1} \\
& =\int \operatorname{sgn}(\widehat{T} f) \circ T \cdot f \mathrm{~d} \mu \leq\|f\|_{1}<\infty .
\end{aligned}
$$

A seguir apresentamos algumas propriedades do operador de transferência, cujas provas podem ser encontradas em [Sa5] Proposição 2.3.

Proposição 21. Sejam $(\Omega, \mathcal{F}, \mu)$ um espaço de medida $\sigma$-finita, $\mu$ não-singular e $T: \Omega \rightarrow \Omega$ uma aplicação mensurável. Logo:

i.) Se $f \in L^{1}(\mu)$ então $\widehat{T} f$ é a única $L^{1}(\mu)$-função tal que para cada $\varphi \in L^{\infty}(\mu)$,

$$
\int \varphi \cdot \widehat{T} f \mathrm{~d} \mu=\int(\varphi \circ T) f \mathrm{~d} \mu .
$$

ii.) Para cada $f \geq 0$, temos $\widehat{T} f \geq 0$.

iii.) O operador de transferência é linear e limitado em $L^{1}(\mu)$. Além disso $\|\widehat{T}\|_{1}=1$.

iv.) Para cada $f \in L^{1}(\mu)$, temos

$$
\int \widehat{T} f \mathrm{~d} \mu=\int f \mathrm{~d} \mu
$$

v.) Para $f \in L^{1}(\mu)$ não-negativa tal que $\int f \mathrm{~d} \mu=1$, então $\widehat{T} f=f$ se, e somente, se $\mathrm{d} m=f \mathrm{~d} \mu$ é $\sigma$-invariante.

A propriedade do operador de transferência dada pela Equação (1.9) é denotada por $\widehat{T}^{*} \mu=\mu$.

A seguinte proposição define ao operador de transferência para um shift de Markov de forma explicita.

Proposição 22. Sejam $\Sigma_{A}$ um shift de Markov e $\mu$ uma medida não-singular em $\mathcal{B}$. Então o operador de transferência para $\sigma$ é dado por

$$
(\widehat{\sigma} f)(x)=\sum_{\sigma y=x} \frac{\mathrm{d} \mu}{\mathrm{d} \mu \odot \sigma}(y) f(y), \quad \mu-\text { q.t.p. },
$$

para cada $f \in L^{1}(\mu)$.

A seguir apresentamos o seguinte corolário que será usado no Capítulo 2.

Corolário 23. Sejam $\Sigma_{A}$ um shift de Markov e $\mu$ uma medida $\sigma$-finita em $\mathcal{B}$. Se $\mu$ é $\sigma$-invariante então

$$
1=\sum_{\sigma y=x} \frac{\mathrm{d} \mu}{\mathrm{d} \mu \odot \sigma}(y), \quad \mu-\text { q.t.p. em } \mathcal{B} .
$$


Demonstração. Pela definição do operador de transferência temos

$$
\hat{\sigma}(\mathbb{1})=\frac{\mathrm{d} \mu \circ \sigma^{-1}}{\mathrm{~d} \mu} .
$$

Como $\mu$ é $\sigma$-invariante então $\mu \circ \sigma^{-1}(E)=\mu(E)$ para todo $E \in \mathcal{B}$. Logo

$$
1=\hat{\sigma}(\mathbb{1}), \quad \mu \text { - q.t.p. em } \mathcal{B} .
$$

Seja $E \in \mathcal{B}$

$$
\int_{E} \hat{\sigma}(\mathbb{1})(x) \mathrm{d} \mu(x)=\int \mathbb{1}_{E}(x) \mathrm{d} \mu \circ \sigma^{-1}(x)=\int \mathbb{1}_{E} \circ \sigma(x) \mathrm{d} \mu(x) .
$$

Usando o item i.) do Lema 16 e o Teorema da Convergência Monótona temos

$$
\begin{aligned}
\int \mathbb{1}_{E} \circ \sigma(x) \mathrm{d} \mu(x) & =\int \mathbb{1}_{E} \circ \sigma(x) \cdot \frac{\mathrm{d} \mu}{\mathrm{d} \mu \odot \sigma}(x) \mathrm{d} \mu \odot \sigma(x) \\
& =\sum_{a \in S} \int_{\sigma[a]}\left(\mathbb{1}_{E} \circ \sigma\right)(a x) \cdot \frac{\mathrm{d} \mu}{\mathrm{d} \mu \odot \sigma}(a x) \mathrm{d} \mu(x) \\
& =\sum_{a \in S} \int \mathbb{1}_{\sigma[a]}(x) \mathbb{1}_{E} \circ \sigma(a x) \cdot \frac{\mathrm{d} \mu}{\mathrm{d} \mu \odot \sigma}(a x) d \mu(x) \\
& =\int \mathbb{1}_{E}(x) \sum_{a \in S} \mathbb{1}_{\sigma[a]}(x) \cdot \frac{\mathrm{d} \mu}{\mathrm{d} \mu \odot \sigma}(a x) \mathrm{d} \mu(x) \\
& =\int_{E} \sum_{\sigma y=x} \frac{\mathrm{d} \mu}{\mathrm{d} \mu \odot \sigma}(y) \mathrm{d} \mu(x) .
\end{aligned}
$$

Das Equações (1.12), (1.13) e (1.14) temos

$$
\int_{E} 1 \mathrm{~d} \mu=\int_{E} \sum_{\sigma y=x} \frac{\mathrm{d} \mu}{\mathrm{d} \mu \odot \sigma}(y) \mathrm{d} \mu(x), \quad \text { para todo } E \in \mathcal{B} .
$$

Logo obtemos a Equação (1.11).

Consideremos $|S|=\infty$ e enumerável. Sejam $\Sigma_{A}$ um shift de Markov e $\phi: \Sigma_{A} \rightarrow \mathbb{R}$ um potencial mensurável. Suponhamos que existe uma medida $\mu$ não-singular e $\sigma$-finita em $\mathcal{B}$ tal que $\mu$ é $(\phi, \lambda)$-conforme, para algum $\lambda>0$. Logo pela Proposição 22 temos que

$$
\sum_{\sigma y=(\cdot)} e^{\phi(y)} f(y) \in L^{1}(\mu)
$$

para cada $f \in L^{1}(\mu)$. Portanto, para cada $f \in L^{1}(\mu)$ o somatório

$$
\sum_{\sigma y=x} e^{\phi(y)} f(y)
$$

converge $\mu$ - q.t.p..

Definição 24 (Operador de Ruelle). Sejam $\Sigma_{A}$ um shift de Markov, $\phi: \Sigma_{A} \rightarrow \mathbb{R}$ um potencial mensurável e $\mu$ uma medida não-singular $\sigma$-finita. Se $\mu$ é $(\phi, \lambda)$-conforme, para algum $\lambda>0$, definimos o operador de Ruelle $L_{\phi}: L^{1}(\mu) \rightarrow L^{1}(\mu)$ por

$$
L_{\phi} f(x)=\sum_{\sigma y=x} e^{\phi(y)} f(y) .
$$


A definição acima mostra a importância de encontrar medidas $\mu$ sendo $(\phi, \lambda)$-conformes em shifts de Markov. No entanto a medida $\mu$ não somente é importante para que o operador de Ruelle esteja bem definido $\mu$ - q.t.p., mas também a importância está em que pelo item $i v$ ) da Proposição 21, $\mu$ é uma automedida do dual do operador de Ruelle $L_{\phi}^{*}(\mu)=\lambda \mu$, isto deve ser entendido no sentido que

$$
\int L_{\phi} f \mathrm{~d} \mu=\lambda \int f \mathrm{~d} \mu \quad \text { para toda } f \in L^{1}(\mu)
$$

Dizemos que um potencial $\phi: \Sigma_{A} \rightarrow \mathbb{R}$ normaliza o operador de Ruelle se, $L_{\phi} \mathbb{1}=1$.

A seguinte Proposição diz que as medidas $(\phi, \lambda)$-conformes são equivalentes às automedidas do dual do operador de Ruelle.

Proposição 25. Sejam $\Sigma_{A}$ um shift de Markov, $\phi: \Sigma_{A} \rightarrow \mathbb{R}$ um potencial mensurável e $\mu$ uma medida $\sigma$-finita. Então $\mu$ é $(\phi, \lambda)$-conforme se, e somente, se

$$
\int L_{\phi} f \mathrm{~d} \mu=\lambda \int f \mathrm{~d} \mu, \quad \text { para cada } f \in L^{1}(\mu),
$$

onde $\lambda>0$.

Demonstração. Seja $E \in \mathcal{B}$. Usando item $i$.) do Lema 16 temos

$$
\begin{aligned}
\lambda^{-1} \int \mathbb{1}_{E}(x) e^{\phi(x)} \mathrm{d} \mu \odot \sigma(x) & =\lambda^{-1} \sum_{a \in S} \int_{\sigma[a]} \mathbb{1}_{E}(a x) e^{\phi(a x)} \mathrm{d} \mu(x) \\
& =\lambda^{-1} \int \sum_{a \in S} \mathbb{1}_{\sigma[a]}(x) \mathbb{1}_{E}(a x) e^{\phi(a x)} \mathrm{d} \mu(x) \\
& =\lambda^{-1} \int \sum_{\sigma(y)=x} \mathbb{1}_{E}(y) e^{\phi(y)} \mathrm{d} \mu(x) \\
& =\lambda^{-1} \int L_{\phi} \mathbb{1}_{E}(x) \mathrm{d} \mu(x) .
\end{aligned}
$$

Logo, para todo $E \in \mathcal{B}$ temos

$$
\lambda^{-1} \int \mathbb{1}_{E}(x) e^{\phi(x)} \mathrm{d} \mu \odot \sigma(x)=\lambda^{-1} \int L_{\phi} \mathbb{1}_{E}(x) \mathrm{d} \mu(x) .
$$

Consideremos $\mu$ uma medida $(\phi, \lambda)$-conforme e $E \in \mathcal{B}$. De (1.17) obtemos

$$
\lambda \mu(E)=\int L_{\phi} \mathbb{1}_{E}(x) \mathrm{d} \mu(x) .
$$

Seja $f \in L^{1}(\mu)$ tal que $f \geq 0$, logo existem funções simples $\left\{s_{n}\right\}_{n \geq 1}$ tais que

$$
0 \leq s_{1}(x) \leq s_{2}(x) \leq \ldots \leq s_{n}(x) \leq \ldots \leq f(x) \quad \text { e } \quad s_{n}(x) \stackrel{n \rightarrow \infty}{\longrightarrow} f(x) .
$$

Notemos que $s_{n} \in L^{1}(\mu)$ para cada $n \in \mathbb{N}$. Usando (1.18) e o Teorema da Convergência Monótona obtemos

$$
\begin{aligned}
\lambda \int f(x) \mathrm{d} \mu(x) & =\lambda \lim _{n \rightarrow \infty} \int s_{n}(x) \mathrm{d} \mu(x) \\
& =\lim _{n \rightarrow \infty} \int L_{\phi} s_{n}(x) \mathrm{d} \mu(x) \\
& =\int \lim _{n \rightarrow \infty} L_{\phi} s_{n}(x) \mathrm{d} \mu(x)
\end{aligned}
$$




$$
=\int L_{\phi} f(x) \mathrm{d} \mu(x) .
$$

No caso em que $f$ toma valores reais basta repetir o argumento apresentado acima para $f^{+}$e $f^{-}$. Notemos que neste caso $f^{+}, f^{-} \in L^{1}(\mu)$ e assim o resultado segue.

Agora consideremos $\mu$ satisfazendo a Equação (1.16). Seja $\left\{A_{i}\right\}_{i \geq 1}$ uma partição mensurável disjunta tal que $\mu\left(A_{i}\right)<\infty$ para cada $i \geq 1$. Para cada $E \in \mathcal{B}$ usando as Equações (1.16) e (1.17) obtemos

$$
\mu\left(E \cap A_{i}\right)=\lambda^{-1} \int_{E \cap A_{i}} e^{\phi(x)} \mathrm{d} \mu \odot \sigma(x) \quad \text { para cada } i \geq 1 .
$$

De (1.19) obtemos

$$
\begin{aligned}
\mu(E) & =\sum_{i \geq 1} \mu\left(E \cap A_{i}\right) \\
& =\lambda^{-1} \sum_{i \geq 1} \int_{E \cap A_{i}} e^{\phi(x)} \mathrm{d} \mu \odot \sigma(x) \\
& =\lambda^{-1} \int_{E} e^{\phi(x)} \mathrm{d} \mu \odot \sigma(x),
\end{aligned}
$$

portanto $\mu$ é uma medida $(\phi, \lambda)$-conforme.

Observação 26. Cada medida conforme é não-singular, em efeito, provaremos isto por contraposição. Consideremos $F \in \mathcal{B}$ tal que $\mu(F)=0$ e $\mu\left(\sigma^{-1} F\right)>0$, note que $\mu \odot \sigma\left(\sigma^{-1} F\right)=0$, logo $\mu$ não satisfaz (1.20). Analogamente suponhamos que $F \in \mathcal{B}$ tal que $\mu(F)>0$ e $\mu\left(\sigma^{-1} F\right)=0$, como $\mu \odot \sigma\left(\sigma^{-1} F\right)>0$ temos que $\mu$ não satisfaz (1.20).

A existência de medidas conformes em um shift de Markov dependem do potencial e do shift de Markov, como mostra o seguinte teorema dado por O. Sarig. A prova é muito técnica e longa por isso apresentaremos um resumo dela por ser um resultado muito importante na teoria de shifts de Markov com alfabeto enumerável e cardinalidade infinita, para os detalhes da prova veja o Teorema $2.9 \mathrm{em}[\mathrm{Sa} 5]$.

Teorema 27. Sejam $\Sigma_{A}$ um shift transitivo e $\phi: \Sigma_{A} \rightarrow \mathbb{R}$ é um potencial que satisfaz a condição de Walters. Então

$$
\begin{aligned}
& \text { i.) } \log \lambda:=\lim \sup _{n \rightarrow \infty} \frac{1}{n} \log Z_{n}(\phi, a)<\infty \text {; } \\
& \text { ii.) } \sum_{n \geq 1} \lambda^{-n} Z_{n}(\phi, a)=\infty
\end{aligned}
$$

onde $Z_{n}(\phi, a)=\sum_{\sigma^{n} x=x} e^{\phi_{n}(x)} \mathbb{1}_{[a]}(x)$ se, e somente, se existe uma medida $\nu$ conservativa ${ }^{2} e$ $(\phi, \beta)$-conforme tal que $0<\nu([a])<\infty$ para cada $a \in S$. Neste caso $\beta=\lambda$.

Demonstração. Consideremos $\lambda<\infty$ e $\sum_{n \geq 1} \lambda^{-n} Z_{n}(\phi, a)=\infty$. Fixemos $a \in S$ e $x_{a} \in[a]$ uma configuração periódica. Para cada $n \in \mathbb{N}$ e $b \in S$ definamos

$$
\begin{aligned}
a_{n} & :=\sum_{k=1}^{n} \lambda^{-k} Z_{k}(\phi, a) \\
\nu_{n}^{b} & :=\frac{1}{a_{n}} \sum_{k=1}^{n} \lambda^{-k} \sum_{\sigma^{k} y=x_{a}} e^{\phi_{k}(y)} \mathbb{1}_{[b]}(y) \delta_{y} .
\end{aligned}
$$

Notemos que $a_{n} \rightarrow \infty$ e $\nu_{n}^{b}$ é uma medida suportada em $[b] \cap \mathcal{B}$. O seguintes fatos podem ser demonstrados:

\footnotetext{
${ }^{2}$ Veja Definição 33.
} 
a.) Para cada $b \in S$ temos

$$
0<\liminf _{n \rightarrow \infty} \nu_{n}^{b}\left(\Sigma_{A}\right) \leq \limsup _{n \rightarrow \infty} \nu_{n}^{b}\left(\Sigma_{A}\right)<\infty
$$

b.) Para cada $b \in S$ e $\varepsilon>0$ existe $K(b, \varepsilon) \subset \Sigma_{A}$ compacto tal que

$$
\nu_{n}^{b}\left(\Sigma_{A} \backslash K(b, \varepsilon)\right)<\varepsilon, \quad \text { para } n \geq 1 .
$$

Estes itens $a$.) e b.) junto com o fato que $\Sigma_{A}$ é um espaço métrico completo e separável garantem as hipóteses do Teorema de Helly-Prohorov, veja Teorema 2.8 in [Sa5]. Assim para cada $b \in S$ existem uma subsequência $\left\{n_{k(b)}\right\}_{k(b) \in \mathbb{N}}$ e uma medida $\nu^{b}$ tais que $\nu_{n_{k(b)}}^{b} \stackrel{*}{\rightarrow} \nu^{b}$. Definamos

$$
\nu:=\sum_{b \in S} \nu^{b}
$$

pela Equação (1.23) e o item a.) temos $0<\nu([a])<\infty$ para cada $a \in S$.

A seguir mostraremos que $L_{\phi}^{*} \nu\left(\mathbb{1}_{[\underline{\omega}]}\right)=\lambda \nu([\underline{\omega}])$, onde $[\underline{\omega}]$ é um cilindro de tamanho arbitrário. Usando as Equações (1.21) e (1.23), para cada $b \in S$ e $N \in \mathbb{N}$ temos

$$
\begin{aligned}
& \nu\left(\mathbb{1}_{\left[x_{0}<N\right]} L_{\phi} \mathbb{1}_{[\underline{\omega}]}\right)=\sum_{b \in S} \nu^{b}\left(\mathbb{1}_{\left[x_{0}<N\right]} L_{\phi} \mathbb{1}_{[\underline{\omega}]}\right) \\
& =\sum_{b \in S} \lim _{n_{k(b)} \rightarrow \infty} \nu_{n_{k(b)}}^{b}\left(\mathbb{1}_{\left[x_{0}<N\right]} L_{\phi} \mathbb{1}_{[\omega]}\right) \\
& =\sum_{b \in S} \lim _{n_{k(b)} \rightarrow \infty} \frac{1}{a_{n_{k(b)}}} \sum_{j \geq 1}^{n_{k(b)}} \lambda^{-j} \sum_{\sigma^{j} y=x_{a}} e^{\phi_{j}(y)} \mathbb{1}_{[b]}(y) \mathbb{1}_{\left[x_{0}<N\right]}(y) L_{\phi} \mathbb{1}_{[\underline{\omega}]}(y) \\
& =\sum_{b \in S} \lim _{n_{k(b)} \rightarrow \infty} \frac{1}{a_{n_{k(b)}}} \sum_{j \geq 1}^{n_{k(b)}} \lambda^{-j}\left(L_{\phi}^{j}\left(\mathbb{1}_{[b]} \mathbb{1}_{\left[x_{0}<N\right]} L_{\phi} \mathbb{1}_{[\underline{\omega}]}\right)\right)\left(x_{a}\right) \\
& =\sum_{b<N} \lim _{n_{k(b)} \rightarrow \infty} \frac{1}{a_{n_{k(b)}}} \sum_{j \geq 1}^{n_{k(b)}} \lambda^{-j}\left(L_{\phi}^{j}\left(\mathbb{1}_{[b]} \mathbb{1}_{\left[x_{0}<N\right]} L_{\phi} \mathbb{1}_{[\omega]}\right)\right)\left(x_{a}\right) \\
& =\lim _{n_{k(b)} \rightarrow \infty} \frac{1}{a_{n_{k(b)}}} \sum_{j \geq 1}^{n_{k(b)}} \lambda^{-j}\left(L_{\phi}^{j}\left(\mathbb{1}_{\left[x_{0}<N\right]} L_{\phi} \mathbb{1}_{[\underline{\omega}]}\right)\right)\left(x_{a}\right) \\
& =\lim _{n_{k(b)} \rightarrow \infty} \frac{1}{a_{n_{k(b)}}} \sum_{j \geq 1}^{n_{k(b)}} \lambda^{-j}\left(L_{\phi}^{j+1}\left(\mathbb{1}_{\left[x_{1}<N\right]} \mathbb{1}_{[\omega]}\right)\right)\left(x_{a}\right) \\
& =\lim _{n_{k(b)} \rightarrow \infty} \frac{\lambda}{a_{n_{k(b)}}} \sum_{j \geq 1}^{n_{k(b)}} \lambda^{-j-1}\left(L_{\phi}^{j+1}\left(\mathbb{1}_{\left[x_{1}<N\right]} \mathbb{1}_{[\underline{\omega}]}\right)\right)\left(x_{a}\right) \\
& =\lim _{n_{k(b)} \rightarrow \infty} \frac{\lambda}{a_{n_{k(b)}}} \sum_{j \geq 1}^{n_{k(b)}} \lambda^{-j} L_{\phi}^{j}\left(\mathbb{1}_{\left[x_{1}<N\right]} \mathbb{1}_{[\underline{\omega}]}\right)\left(x_{a}\right) \\
& +\lim _{n_{k(b)} \rightarrow \infty} \frac{\lambda}{a_{n_{k(b)}}} \lambda^{-n_{k(b)}-1} L_{\phi}^{n_{k(b)}+1}\left(\mathbb{1}_{\left[x_{1}<N\right]} \mathbb{1}_{[\underline{\omega}]}\right)\left(x_{a}\right) \\
& -\lim _{n_{k(b)} \rightarrow \infty} \frac{\lambda}{a_{n_{k(b)}}} \lambda^{-1} L_{\phi}\left(\mathbb{1}_{\left[x_{1}<N\right]} \mathbb{1}_{[\underline{\omega}]}\right)\left(x_{a}\right) \\
& =\lambda \lim _{n_{k(b)} \rightarrow \infty} \frac{1}{a_{n_{k(b)}}} \sum_{j \geq 1}^{n_{k(b)}} \lambda^{-j} L_{\phi}^{j}\left(\mathbb{1}_{\left[x_{1}<N\right]} \mathbb{1}_{[\omega]}\right)\left(x_{a}\right) \\
& =\lambda \nu\left(\mathbb{1}_{\left[x_{1}<N\right]} \mathbb{1}_{[\underline{\omega}]}\right) \text {. }
\end{aligned}
$$


Portanto para cada $b \in S$ e $[\underline{\omega}]$ cilindro de tamanho arbitrário temos

$$
\nu\left(\mathbb{1}_{\left[x_{0}<N\right]} L_{\phi} \mathbb{1}_{[\underline{\omega}]}\right)=\lambda \nu\left(\mathbb{1}_{\left[x_{1}<N\right]} \mathbb{1}_{[\underline{\omega}]}\right) .
$$

Seja $N \in \mathbb{N}$, denotemos $g_{N}:=\mathbb{1}_{\left[x_{0}<N\right]}$ e $h_{N}:=\mathbb{1}_{\left[x_{1}<N\right]}, \operatorname{logo} g_{N} \stackrel{N \rightarrow \infty}{\longrightarrow} \mathbb{1}$ e $h_{N} \stackrel{N \rightarrow \infty}{\longrightarrow} \mathbb{1}$. Usando a Equação (1.24) e o Teorema da Convergência Monótona temos

$$
\begin{aligned}
\nu\left(L_{\phi} \mathbb{1}_{[\underline{\omega}]}\right) & =\lim _{N \rightarrow \infty} \nu\left(g_{N} L_{\phi} \mathbb{1}_{[\underline{\omega}]}\right) \\
& =\lim _{N \rightarrow \infty} \lambda \nu\left(h_{N} \mathbb{1}_{[\underline{\omega}]}\right) \\
& =\lambda \nu([\underline{\omega}]) .
\end{aligned}
$$

Como $[\underline{\omega}]$ é um cilindro de tamanho arbitrário e a coleção de todos os cilindros é a base da topologia, logo pelo fato de $\nu$ ser uma medida regular temos que

$$
\nu\left(L_{\phi} f\right)=\lambda \nu(f), \quad \text { para cada } f \in L^{1}(\nu) .
$$

Pela Equação (1.25) e a Proposição 25 temos que $\nu$ é $(\phi, \lambda)$-conforme. Além disso pelo Teorema $2.2 \mathrm{em}[\mathrm{Sa} 5]$ temos que $\nu$ é conservativa.

Agora suponhamos que $\nu$ é uma medida conservativa e $(\phi, \beta)$-conforme. Pode-se mostrar que $\lambda \leq \beta, \operatorname{logo} \log \lambda<\infty$. Como $\sum_{n \geq 1} \beta^{-n} Z_{n}(\phi, a)=+\infty$, veja o Teorema 2.2 em [Sa5], e

$$
\sum_{n \geq 1} \beta^{-n} Z_{n}(\phi, a) \leq \sum_{n \geq 1} \lambda^{-n} Z_{n}(\phi, a)
$$

logo temos o item $i i$.).

O Teorema 27 fornece condições para que o operador de Ruelle esteja bem definido. Porém podese impor outros tipos de regularidades no potencial ou no shift de Markov. A seguir apresentamos alguns casos:

a.) Sejam $\Sigma_{A}$ um shift de Markov transitivo e localmente compacto, e $\phi: \Sigma_{A} \rightarrow \mathbb{R}$ um potencial contínuo. O operador de Ruelle pode ser definido no conjunto de funções contínuas com suporte compacto $C_{c}\left(\Sigma_{A}\right)$, isto é,

$$
L_{\phi}: C_{c}\left(\Sigma_{A}\right) \rightarrow C_{c}\left(\Sigma_{A}\right)
$$

Note que $L_{\phi}$ está bem definido. De fato, seja $f \in C_{c}\left(\Sigma_{A}\right)$ então existem $b_{1}, b_{2}, \ldots, b_{N} \in S$ tal que $\operatorname{supp}(f) \subset \cup_{i=1}^{N}\left[b_{i}\right]$, logo:

i.) $L_{\phi}$ é contínuo. Seja $x \in \Sigma_{A}$. Dado $\varepsilon=2^{-n}, n \in \mathbb{N}$, para cada $i \in\{1,2, \ldots, N\}$ satisfazendo $A\left(b_{i}, x_{0}\right)=1$ existe $\delta_{i}>0$ tal que

$$
\left|e^{\phi\left(b_{i} x\right)} f\left(b_{i} x\right)-e^{\phi(z)} f(z)\right|<\varepsilon N
$$

para todo $z \in \Sigma_{A}$ tal que $d\left(b_{i} x, z\right)<\delta_{i}$. Definindo $\delta:=\min \left\{\delta_{i}: i=1, \ldots, N\right\}$, por (1.26) para cada $y \in \Sigma_{A}$ tal que $d(x, y)<\delta$ temos

$$
\begin{aligned}
\left|L_{\phi} f(x)-L_{\phi} f(y)\right| & =\left|\sum_{\xi \in S \mid A\left(\xi, x_{0}\right)=1} e^{\phi(\xi x)} f(\xi x)-e^{\phi(\xi y)} f(\xi y)\right| \\
& \leq \sum_{\xi \in S \mid A\left(\xi, x_{0}\right)=1}\left|e^{\phi(\xi x)} f(\xi x)-e^{\phi(\xi y)} f(\xi y)\right|
\end{aligned}
$$




$$
\begin{aligned}
& \leq \sum_{i=1 \mid A\left(b_{i}, x_{0}\right)=1}^{N}\left|e^{\phi\left(b_{i} x\right)} f\left(b_{i} x\right)-e^{\phi\left(b_{i} y\right)} f\left(b_{i} y\right)\right| \\
& <\varepsilon .
\end{aligned}
$$

ii.) $L_{\phi} f \in C_{c}\left(\Sigma_{A}\right)$. Como $f$ tem suporte compacto temos

$$
\begin{aligned}
L_{\phi} f(x) & =\sum_{\sigma y=x} e^{\phi(y)} f(y) \\
& \leq \sum_{\sigma y=x} \sum_{i=1}^{N} e^{\phi(y)} \max _{\xi \in\left[b_{i}\right]} f(\xi) \cdot \mathbb{1}_{\left[b_{i}\right]}(y) \\
& \leq \sum_{i=1 \mid A\left(b_{i}, x_{0}\right)=1}^{N} e^{\phi\left(b_{i} x\right)} \max _{\xi \in\left[b_{i}\right]} f(\xi) .
\end{aligned}
$$

Pelas Proposições 1 e 4 temos que $\sum_{b \in S} A(b, a)<\infty$, para cada $a \in S$, logo (1.27) é zero fora de uma união finita de cilindros. Portanto $L_{\phi} f$ tem suporte compacto.

b.) Consideremos $\Sigma_{A}$ um shift de Markov e $\phi: \Sigma_{A} \rightarrow \mathbb{R}$ um potencial somável. Note que

$$
L_{\phi}: C_{b}\left(\Sigma_{A}\right) \rightarrow C_{b}\left(\Sigma_{A}\right)
$$

está bem definido. Além disso, se $\Sigma_{A}$ é um shift transitivo e $\phi$ é localmente Hölder então existe $\nu$ medida de probabilidade tal que $L_{\phi}^{*} \nu=\lambda \nu$, veja Lema 2.8 em [MaUr1], onde $\lambda$ é dado pelo item $i$.) do Teorema 27.

c.) Note que na definição do Operador de Ruelle do item b.) a hipótese do potencial pode ser enfraquecida pedindo que $\left\|L_{\phi} \mathbb{1}\right\|_{\infty}<\infty$.

\subsubsection{Pressão de Gurevich}

Nesta subseção apresentamos a definição da Pressão de Gurevich, este conceito é importante por sua relação com as medidas de equilíbrio, veja a Seção 1.5.

Definição 28. Sejam $\Sigma_{A}$ um shift topologicamente mixing e $\phi: \Sigma_{A} \rightarrow \mathbb{R}$ um potencial que satisfaz a condição de Walters. A pressão de Gurevich de $\phi$ é definida por

$$
P_{G}(\phi):=\lim _{n \rightarrow \infty} \frac{1}{n} \log Z_{n}(\phi, a)
$$

onde $Z_{n}(\phi, a)=\sum_{\sigma^{n} x=x} e^{\phi_{n}(x)} \mathbb{1}_{[a]}(x)$.

Nas condições da Definição 28, o limite da Equação (1.28) existe para todo $a \in S$, não depende de $a \in S$ e $-\infty<P_{G}(\phi) \leq \infty$, veja [Sa5]. A pressão de Gurevich tem um papel importante na teoria das medidas de equilíbrio, citaremos alguns resultados importantes desta na Seção 1.5.

Definição 29. Sejam $\phi, \psi: \Sigma_{A} \rightarrow \mathbb{R}$ dois potenciais. Dizemos que $\phi$ e $\psi$ são cohomólogos através da função contínua $h: \Sigma_{A} \rightarrow \mathbb{R}$ se $\phi=\psi+h-h \circ \sigma$ e denotaremos por $\phi \sim \psi$.

A seguir temos algumas propriedades da pressão de Gurevich e a prova os detalhes da prova veja Proposição 4.4 em [Sa5].

Proposição 30. Sejam $\Sigma_{A}$ um shift topologicamente mixing e $\phi, \psi: \Sigma_{A} \rightarrow \mathbb{R}$ dois potenciais satisfazendo a condição de Walters, então a pressão de Gurevich tem as seguintes propriedades:

i) Adição de constante: Para cada $c \in \mathbb{R}$ temos $P_{G}(\phi+c)=P_{G}(\phi)+c$. 
ii) Convexidade: Para $t \in[0,1]$ temos $P_{G}(t \phi+(1-t) \psi) \leq t P_{G}(\phi)+(1-t) P_{G}(\psi)$.

iii) Cohomologia: Se $\phi \sim \psi$ então $P_{G}(\phi)=P_{G}(\psi)$.

A seguir temos mais propriedades da pressão de Gurevich envolvendo o operador de Ruelle, para os detalhes da prova veja a Proposição 3.2 em [Sa5].

Proposição 31. Seja $\Sigma_{A}$ um shift topologicamente mixing e $\phi: \Sigma_{A} \rightarrow \mathbb{R}$ um potencial que satisfaz a condição de Walters, logo

i.) $S e\left\|L_{\phi} \mathbb{1}\right\|_{\infty}<\infty$, então $P_{G}(\phi)<\infty$.

ii) Se $P_{G}(\phi)<\infty$, então $L_{\phi}^{n} \mathbb{1}_{[a]}$ é finito para cada $n \in \mathbb{N}$ e $a \in S$.

iii.) Para cada função contínua e limitada $f$ não negativa, não identicamente igual a zero e suportada dentro de uma união finita de cilindros, temos

$$
P_{G}(\phi)=\lim _{n \rightarrow \infty} \frac{1}{n} \log \left(L_{\phi}^{n} f\right)(x), \quad \text { para cada } x \in \Sigma_{A} .
$$

Pelo item $i$.) temos que a condição $\left\|L_{\phi} \mathbb{1}\right\|_{\infty}<\infty$ garante que $P_{G}(\phi)<\infty$. Outra condição para garantir isso é que o potencial seja somável, isto é, todo potencial somável tem pressão de Gurevich finita. Para isso é suficiente notar que

$$
L_{\phi} \mathbb{1}(x)=\sum_{\sigma y=x} e^{\phi(y)} \leq \sum_{a \in S} e^{\left.\sup \phi\right|_{[a]}}<\infty .
$$

A recíproca é válida quando o $\Sigma_{A}$ satisfaz a propriedade $B I P$, veja [Pe].

Proposição 32. Sejam $\Sigma_{A}$ um shift topologicamente transitivo e $\phi: \Sigma_{A} \rightarrow \mathbb{R}$ um potencial somável tal que a $P_{G}(\phi)$ existe. Seja $H(\phi):=\sum_{i \geq 1} e^{\left.\sup \phi\right|_{[i]}}<\infty$ então $P_{G}(\phi)<\log H(\phi)$.

Demonstração. Denotemos $H_{n}(\phi):=\sum_{\underline{\omega} \in \mathcal{W}_{n}} e^{\left.\left.\sup \phi_{n}\right|_{[\underline{ }}\right]}$. A seguir provaremos que

$$
H_{n}(\phi) \leq H^{n}(\phi), \quad \text { para todo } n \in \mathbb{N} .
$$

De fato, note que

$$
\begin{aligned}
H_{n}(\phi) & =\sum_{\underline{\omega} \in \mathcal{W}_{n}} e^{\sup \phi_{n} \mid[\underline{\omega}]} \\
& =\sum_{\underline{\omega} \in \mathcal{W}_{n}} e^{\sup _{x \in[\underline{\omega}]}\left(\sum_{i=0}^{n-1} \phi\left(\sigma^{i} x\right)\right)} \\
& \leq \sum_{\underline{\omega} \in \mathcal{W}_{n}} e^{\left(\sum_{i=0}^{n-1} \sup _{x \in[\underline{\omega}]} \phi\left(\sigma^{i} x\right)\right)} \\
& =\sum_{\underline{\omega} \in \mathcal{W}_{n}}\left(e^{\sup _{x \in[\underline{\omega}]} \phi(x)}\right)\left(e^{\sup _{x \in[\underline{\omega}]} \phi(\sigma x)}\right) \ldots\left(e^{\sup _{x \in[\underline{\omega}]} \phi\left(\sigma^{n-1} x\right)}\right) \\
& \leq \sum_{\underline{\omega} \in \mathcal{W}_{n}}\left(e^{\sup _{x \in[\underline{\omega}]} \phi(x)}\right)\left(e^{\sup _{x \in \sigma[\underline{\omega}]} \phi(x)}\right) \ldots\left(e^{\sup _{x \in \sigma^{n-1}[\underline{\omega}]} \phi(x)}\right)
\end{aligned}
$$




$$
\begin{aligned}
& =\sum_{\substack{w_{0}, w_{1}, \ldots, w_{n-1} \in S \\
A\left(w_{0}, w_{1}\right) \ldots A\left(w_{n-2}, w_{n-1}\right)=1}}\left(e^{\sup _{x \in\left[w_{0}, \ldots w_{n-1}\right]} \phi(x)}\right)\left(e^{\sup ^{x \in\left[w_{1}, \ldots, w_{n-1}\right]} \phi(x)}\right) \ldots\left(e^{\sup _{x \in\left[w_{n-1}\right]} \phi(x)}\right) \\
& \leq \quad \sum_{\substack{w_{0}, w_{1}, \ldots, w_{n-1} \in S \\
A\left(w_{0}, w_{1}\right) \ldots A\left(w_{n-2}, w_{n-1}\right)=1}}\left(e^{\sup _{x \in\left[w_{0}\right]} \phi(x)}\right)\left(e^{\sup _{x \in\left[w_{1}\right]} \phi(x)}\right) \ldots\left(e^{\sup _{x \in\left[w_{n-1}\right]} \phi(x)}\right) \\
& \leq \sum_{w_{0}, w_{1}, \ldots, w_{n-1} \in S}\left(e^{\sup _{x \in\left[w_{0}\right]} \phi(x)}\right)\left(e^{\sup _{x \in\left[w_{1}\right]} \phi(x)}\right) \ldots\left(e^{\sup _{x \in\left[w_{n-1}\right]} \phi(x)}\right) \\
& \leq \sum_{w_{0} \in S}\left(e^{\sup _{x \in\left[w_{0}\right]} \phi(x)}\right) \sum_{w_{1} \in S}\left(e^{\sup _{x \in\left[w_{1}\right]} \phi(x)}\right) \cdots \sum_{w_{n-1} \in S}\left(e^{\sup _{x \in\left[w_{n-1}\right]} \phi(x)}\right) \\
& =H^{n}(\phi) \text {. }
\end{aligned}
$$

Notemos que para cada $n \in \mathbb{N}$, temos

$$
\begin{aligned}
Z_{n}(\phi, a) & =\sum_{\sigma^{n} x=x} e^{\phi_{n}(x)} \mathbb{1}_{[a]}(x) \leq \sum_{\sigma^{n} x=x} e^{\phi_{n}(x)} \\
& \leq \sum_{\left\{w \in \Sigma_{A} \mid \sigma^{n} w=w\right\}} e^{x \in\left[w_{0}^{n-1}\right]} \phi_{n}(x) \\
& \leq H_{n}(\phi) .
\end{aligned}
$$

De (1.30) e (1.31) concluímos que $P_{G}(\phi) \leq \log H(\phi)$.

\subsubsection{Teorema de Ruelle-Perron-Frobenius Generalizado}

O famoso Teorema de Ruelle-Perron-Frobenius foi provado por D. Ruelle em [Ru68], este afirma que para shifts de Markov topologicamente mixing com alfabeto finito e potenciais localmente Hölder existem uma automedida de probabilidade $\nu$ para o dual do operador de Ruelle e uma função contínua e positiva $h$ tal que $\int h \mathrm{~d} \nu=1$, para mais detalhes veja [Bo74] e [Ru68]. Nesta subseção apresentaremos o análogo ao Teorema de Ruelle-Perron-Frobenius para shifts de Markov topologicamente mixing com alfabeto infinito, este foi provado por O. Sarig em [Sa1]. Trabalhos nesta direção também foram abordados por V. Cyr, O. Shwartz e M. Urbański em [Cyr1], [Sw] e [MaUr1], respectivamente.

Definição 33. Sejam $(\Omega, \mathcal{F}, \mu)$ um espaço de medida $\sigma$-finito e $T: \Omega \rightarrow \Omega$ uma aplicação nãosingular. A medida $\mu$ é chamada conservativa se para cada $A \in \mathcal{F}$ tal que $\left\{T^{-n} A\right\}_{n \geq 0}$ são dois a dois disjuntos, $\mu$ - q.t.p., temos que $\mu(A)=0$.

As medidas conservativas são as medidas $\sigma$-finitas que satisfazem o Teorema de Recorrência de Poincaré, veja o Teorema $2.1 \mathrm{em}$ [Sa5]. As automedidas de probabilidade que existem para o dual do operador de Ruelle no caso de alfabeto finito são conservativas, veja o Corolário 2.2 em [Sa5]. De maneira análoga temos para o caso de alfabeto infinito enumerável como mostra o Teorema 36. A seguir definiremos os modos de recorrência do potencial, estes caracterizam a existência de medidas conformes conservativas.

Dado um potencial $\phi: \Sigma_{A} \rightarrow \mathbb{R}$ para cada $n \in \mathbb{N}$ e $a \in S$ definimos as seguintes funções de partições:

$$
Z_{n}(\phi, a):=\sum_{\sigma^{n} x=x} \mathbb{1}_{[a]}(x) e^{\phi_{n}(x)} \quad \text { e } Z_{n}^{*}(\phi, a):=\sum_{\substack{\sigma^{n} x=x \\ x_{1}, \ldots, x_{n-1} \neq a}} \mathbb{1}_{[a]}(x) e^{\phi_{n}(x)}
$$


Definição 34. Sejam $\Sigma_{A}$ um shift topologicamente mixing e $\phi: \Sigma_{A} \rightarrow \mathbb{R}$ um potencial que satisfaz a condição de Walters tal que $P_{G}(\phi)<\infty$. Sejam $\lambda:=e^{P_{G}(\phi)}$ e a $\in$ S dizemos que o potencial $\phi e ́$

i) Recorrente, se $\sum_{n \geq 1} \lambda^{-n} Z_{n}(\phi, a)=\infty$;

ii) Positivamente recorrente, se é recorrente e $\sum_{n \geq 1} n \lambda^{-n} Z_{n}^{*}(\phi, a)<\infty$;

iii) Nulamente recorrente, se é recorrente e $\sum_{n \geq 1} n \lambda^{-n} Z_{n}^{*}(\phi, a)=\infty$;

iv) Transiente, se $\sum_{n \geq 1} \lambda^{-n} Z_{n}(\phi, a)<\infty$.

Cada modo de recorrência não depende do símbolo $a \in S$. O Teorema Ruelle-Perron-Frobenius Generalizado (Teorema 36) caracteriza cada um destes potenciais. A seguinte proposição dá condições suficientes para que um potencial seja positivamente recorrente.

Proposição 35 ([Sa5])). Sejam $\Sigma_{A}$ um shift topologicamente mixing satisfazendo a propriedade BIP e $\phi: \Sigma_{A} \rightarrow \mathbb{R}$ um potencial que satisfaz a condição de Walters tal que $P_{G}(\phi)<\infty$. Se $\operatorname{Var}_{1} \phi<\infty$ então $\phi$ é positivamente recorrente.

Note que quando $\Sigma_{A}$ é o full shift com alfabeto $\mathbb{N}$ e $\phi: \Sigma_{A} \rightarrow \mathbb{R}$ um potencial que satisfaz a condição de Walters tal que normaliza o operador de Ruelle temos que $\phi$ é positivamente recorrente quando $\operatorname{Var}_{1}(\phi)<\infty$, pois neste caso $\Sigma_{A}$ tem a propriedade $B I P$ e $P_{G}(\phi) \leq 0$.

Considerando $\Sigma_{A}$ um shift de Markov transitivo com potencial $\phi: \Sigma_{A} \rightarrow \mathbb{R}$ temos que o potencial é positivamente recorrente quando consideramos um potencial somável tais que $\sum_{n \geq 1} \operatorname{Var}_{n} \phi<$ $\infty$ e $P_{G}(\phi)<\infty$ veja $[\mathrm{FrV}]$ e $[\mathrm{Va}]$.

Teorema 36 (Teorema de Ruelle-Perron-Frobenius Generalizado, [Sa2]). Sejam $\Sigma_{A}$ um shift topologicamente mixing e $\phi: \Sigma_{A} \rightarrow \mathbb{R}$ um potencial que satisfaz a condição de Walters tal que $P_{G}(\phi)<\infty$. Então:

1.) $\phi$ é recorrente se, e somente, se existem $\lambda>0, h$ função não-negativa e contínua e $\nu$ uma medida conservativa finita em cilindros tais que $L_{\phi} h=\lambda h, L_{\phi}^{*} \nu=\lambda \nu$. Neste caso $\lambda=e^{P_{G}(\phi)}$.

2.) $\phi$ é positivamente recorrente se, e somente, se existem $\lambda>0, h$ função não-negativa e contínua e $\nu$ uma medida conservativa finita em cilindros tais que $L_{\phi} h=\lambda h, L_{\phi}^{*} \nu=\lambda \nu e$ $\int h \mathrm{~d} \nu<\infty$. Neste caso $\lambda=e^{P_{G}(\phi)}$. Além disso, para cada cilindro $[a]$ temos

$$
\lambda^{-n} L_{\phi}^{n} \mathbb{1}_{[a]} \stackrel{n \rightarrow \infty}{\longrightarrow} \frac{h \nu[a]}{\int h \mathrm{~d} \nu},
$$

pontualmente em $\Sigma_{A}$ e uniformemente em compactos.

3.) $\phi$ é nulamente recorrente se, e somente, se existem $\lambda>0, h$ função não-negativa e contínua e $\nu$ uma medida conservativa finita em cilindros tais que $L_{\phi} h=\lambda h, L_{\phi}^{*} \nu=\lambda \nu$ e $\int h d \nu=\infty$. Neste caso $\lambda=e^{P_{G}(\phi)}$. Além disso, para cada cilindro $[a]$ temos $\lambda^{-n} L_{\phi}^{n} \mathbb{1}_{[a]} \rightarrow 0$ uniformemente em compactos.

4.) $\phi$ é transiente se, e somente, se não existe uma medida conservativa $\nu$ finita em cilindros tal que $L_{\phi}^{*} \nu=\lambda \nu$ para algum $\lambda>0$.

Sob as hipóteses do teorema acima para um shift de Markov com alfabeto finito temos que o potencial sempre é positivamente recorrente e assim o teorema acima é reduzido somente ao item 2.).

Notemos que quando $\phi$ é positivamente recorrente O. Sarig dá uma forma explícita para a autofunção $h$, dada por (1.32). Além disso, sob as hipóteses do Teorema acima com a condição de 
$\Sigma_{A}$ ter a propriedade $B I P$ obtemos que $h$ está afastada uniformemente de zero e do infinito, veja [Sa5].

A seguir apresentamos um exemplo de um shift topologicamente mixing e uma família de potenciais positivamente recorrentes tais que as automedidas associadas podem ser finitas ou infinitas.

Exemplo 37. Consideremos $\Sigma_{A}$ o shift renewal e $\phi: \Sigma_{A} \rightarrow \mathbb{R}$ o potencial dado por $\phi(x)=x_{0}-x_{1}$ e $\beta>0$.

Notemos que $\Sigma_{A}$ é topologicamente mixing, $\phi$ satisfaz a condição de Walters e $\left\|L_{\phi} \mathbb{1}\right\|_{\infty}<\infty$. Além disso, para cada $\beta>0$ temos

$$
\begin{aligned}
P_{G}(\beta \phi) & =\lim _{n \rightarrow \infty} \frac{1}{n} \log \left(\sum_{\sigma^{n} x=x} e^{\beta \phi_{n}(x)} \mathbb{1}_{[1]}(x)\right) \\
& =\lim _{n \rightarrow \infty} \frac{1}{n} \log 2^{n-1} \\
& =\log 2 .
\end{aligned}
$$

Seja $\lambda:=e^{P_{G}(\beta \phi)}=2$, notemos que

$$
\begin{aligned}
\sum_{n \geq 1} \lambda^{-n} Z_{n}(\beta \phi, 1) & =\sum_{n \geq 1} 2^{-n} \#\left\{x \in \Sigma_{A} \mid \sigma^{n} x=x, x_{0}=1\right\} \\
& =\sum_{n \geq 1} 2^{-1}=+\infty
\end{aligned}
$$

$e$

$$
\sum_{n \geq 1} n \lambda^{-n} Z_{n}^{*}(\beta \phi, 1)=\sum_{n \geq 1} n 2^{-n}<+\infty
$$

Logo $\beta \phi$ é positivamente recorrente para todo $\beta>0$.

Fixado $\beta>0$, pelo Teorema de Ruelle-Perron-Frobenius Generalizado existe uma automedida $\nu_{\beta}$ tal que

$$
\int L_{\phi} f \mathrm{~d} \nu_{\beta}=\lambda \int f \mathrm{~d} \nu_{\beta}, \quad \text { para } f \in L^{1}\left(\nu_{\beta}\right) .
$$

Para cada $a \geq 2$, considerando $f:=\mathbb{1}_{[a]}$, substituindo em (1.33) temos

$$
\begin{aligned}
2 \nu_{\beta}([a]) & =\int_{\Sigma_{A}} \sum_{\sigma y=x} e^{\beta \phi(y)} \mathbb{1}_{[a]}(y) \mathrm{d} \nu_{\beta}(x) \\
& =\int_{[a-1]} \sum_{\sigma y=x} e^{\beta \phi(y)} \mathbb{1}_{[a]}(y) \mathrm{d} \nu_{\beta}(x) \\
& =e^{\beta} \nu_{\beta}([a-1]) .
\end{aligned}
$$

$\operatorname{Logo} \nu_{\beta}([a])=\frac{e^{\beta}}{2} \nu_{\beta}([a-1])$ para cada $\beta>0$ e a $\geq 2$, e

$$
\nu_{\beta}\left(\Sigma_{A}\right)=\nu_{\beta}([1])\left(\sum_{k \geq 0}\left(\frac{e^{\beta}}{2}\right)^{k}\right) .
$$

Notemos que a automedida $\nu_{\beta}$ é finita para $\beta \in(0, \log 2)$ e infinita para cada $\beta \in[\log 2,+\infty)$.

Definição 38 (Medida RPF). Sejam $\Sigma_{A}$ um shift topologicamente mixing e $\phi$ um potencial que satisfaz a condição de Walters tal que $P_{G}(\phi)<\infty$. Quando $\phi$ é positivamente recorrente e $\int h d \nu=1$ chamaremos a $\rho:=h d \nu$ de medida $\mathrm{RPF}$. 
A medida de RPF é de probabilidade e $\sigma$-invariante, veja [Sa5]. Além disso é única, para ver isso é suficiente mostrar que o espaço das autofunções e automedidas associadas ao operador de Ruelle é unidimensional.

Proposição 39. Sejam $\Sigma_{A}$ um shift topologicamente mixing e $\phi: \Sigma_{A} \rightarrow \mathbb{R}$ um potencial que satisfaz a condição de Walters tal que $P_{G}(\phi)<\infty$. Se $\phi$ é positivamente recorrente então a família de autofunções e automedidas associadas a $e^{P_{G}(\phi)}$ é unidimensional.

Demonstração. De fato, sejam $\nu$ e $\mu$ automedidas associadas as autofunções $h$ e $f$ respectivamente. De (1.32) temos que $\frac{\nu[a]}{\mu[a]}$ é constante para todo $a \in S$. Em efeito para cada $x \in \Sigma_{A}$ e $a \in S$ temos

$$
\begin{aligned}
& \lambda^{-n} L_{\phi}^{n} \mathbb{1}_{[a]}(x) \stackrel{n \rightarrow \infty}{\longrightarrow} \frac{h(x) \nu[a]}{\int h \mathrm{~d} \nu}, \\
& \lambda^{-n} L_{\phi}^{n} \mathbb{1}_{[a]}(x) \stackrel{n \rightarrow \infty}{\longrightarrow} \frac{f(x) \mu[a]}{\int f \mathrm{~d} \mu},
\end{aligned}
$$

logo de (1.34) e (1.35) temos que $\frac{h(x)}{f(x)}=\frac{\nu[a]}{\mu[a]}$. Fixando $x \in \Sigma_{A}$ e e repetindo (1.34) e (1.35) para $b \in S$ obtemos que

$$
\frac{\nu[a]}{\mu[a]}=\frac{\nu[b]}{\mu[b]}, \quad \forall a, b \in S .
$$

Portanto temos $f=$ cte.h. Como $f=$ cte.h obtemos de maneira análoga $\mu[a]=\nu[a] \frac{\int h \mathrm{~d} \mu}{\int h \mathrm{~d} \nu}$ para todo $a \in S$, isso pode ser estendido para a álgebra gerada pelos cilindros. O Teorema de extensão de Caratheodory estende isso para a $\sigma$-álgebra de Borel.

Há uma maneira padrão de normalizar o operador de Ruelle. Sejam $\Sigma_{A}$ um shift topologicamente mixing e $\phi: \Sigma_{A} \rightarrow \mathbb{R}$ um potencial recorrente. Sob as hipóteses do Teorema 36, definindo:

$$
\widetilde{\phi}:=\phi+\log h-\log h \circ \sigma-P_{G}(\phi)
$$

onde $h$ é a autofunção do operador de Ruelle, temos que $\widetilde{\phi}: \Sigma_{A} \rightarrow \mathbb{R}$ normaliza o operador de Ruelle.

No caso quando $\Sigma_{A}$ é topologicamente mixing satisfazendo a propriedade $B I P, \phi$ é um potencial com variação somável e $P_{G}(\phi)<\infty$ obtemos que $\phi$ é positivamente recorrente, veja [Sa4]. Assim neste caso o operador de Ruelle pode ser normalizado.

O Teorema de Ruelle-Perron-Frobenius Generalizado garante a existência de medidas conformes conservativas para potencias recorrentes, mas não para potenciais transientes. V. Cyr faz um estudo detalhado de shifts de Markov associados a potenciais transientes. Um resultado importante obtido nesta direção é o Teorema 46 dado por V. Cyr e O. Shwartz, que é o análogo ao Teorema de RuellePerron-Frobenius mas para potencias transientes. A seguir detalharemos esta abordagem, para mais detalhes veja [Cyr1] e $[\mathrm{Sw}]$.

Definição 40. Seja $\Sigma_{A}$ um shift transitivo localmente compacto e $\phi: \Sigma_{A} \rightarrow \mathbb{R}$ um potencial que satisfaz a condição de Walters. Para cada $f \in C_{c}^{+}\left(\Sigma_{A}\right)$ e $\lambda>0$, a função de Green para $f$ e $\lambda$ em $x \in \Sigma_{A}$ é definida por

$$
G(f, x \mid \lambda):=\sum_{n \geq 0} \lambda^{-n}\left(L_{\phi}^{n} f\right)(x) .
$$

Dizemos que um potencial $\phi: \Sigma_{A} \rightarrow \mathbb{R}$ é $\lambda$-transiente quando $\sum_{n \geq 1} \lambda^{-n} Z_{n}(\phi, a)<+\infty$ para algum $a \in S$. Pode-se provar que $\phi$ é $\lambda$-transiente se, e somente, se $G\left(\mathbb{1}_{[a]}, x \mid \lambda\right)<+\infty$ para algum $a \in S$ e $x \in \Sigma_{A}$. 
Definição 41. Sejam $\Sigma_{A}$ um shift transitivo localmente compacto e $\phi: \Sigma_{A} \rightarrow \mathbb{R}$ um potencial $\lambda$-transiente que satisfaz a condição de Walters. Seja o $\in S$ um estado arbitrário, o Kernel de Martin $K: C_{c}\left(\Sigma_{A}\right) \times \Sigma_{A} \rightarrow \mathbb{R}$ é definido

$$
K(f, x \mid \lambda):=\frac{G(f, x \mid \lambda)}{G\left(\mathbb{1}_{[o]}, x \mid \lambda\right)} .
$$

Na Proposição 43 mostraremos que $K(f, x \mid \lambda)$ está bem definido, isto é, $K(f, \cdot \mid \lambda)$ é finito para cada $x \in \Sigma_{A}$. Primeiro provaremos o seguinte lema.

Lema 42. Sejam $\Sigma_{A}$ um shift transitivo localmente compacto e $\phi: \Sigma_{A} \rightarrow \mathbb{R}$ um potencial $\lambda$-transiente satisfazendo a condição de Walters. Então, para cada $a, b \in S$ existem $0<c_{a, b} \leq$ $C_{a, b}<\infty$ tais que

$$
c_{a, b} G\left(\mathbb{1}_{[b]}, x \mid \lambda\right) \leq G\left(\mathbb{1}_{[a]}, x \mid \lambda\right) \leq C_{a, b} G\left(\mathbb{1}_{[b]}, x \mid \lambda\right), \quad \forall x \in \Sigma_{A} .
$$

Demonstração. Como $\Sigma_{A}$ é transitivo existem $N \in \mathbb{N}$ e $\xi_{1}, \ldots, \xi_{N-1} \in S$ tais que $A\left(b, \xi_{1}\right)=1$, $A\left(\xi_{1}, \xi_{2}\right)=1, \ldots, A\left(\xi_{N-1}, a\right)=1$. Seja $x \in \Sigma_{A}, \operatorname{logo}$

$$
\begin{aligned}
G\left(L_{\phi}^{N} \mathbb{1}_{[b]}, x \mid \lambda\right) & =\sum_{n \geq 0} \lambda^{-n} L_{\phi}^{n+N} \mathbb{1}_{[b]}(x) \\
& =\lambda^{N} \sum_{n \geq N} \lambda^{-n} L_{\phi}^{n} \mathbb{1}_{[b]}(x) \\
& \leq \lambda^{N} G\left(\mathbb{1}_{[b]}, x \mid \lambda\right) .
\end{aligned}
$$

Consideremos $M_{k}:=\sup _{n \geq 1} \operatorname{Var}_{n+k} \phi_{n}$. Fixando $x_{a} \in[a]$ e usando (1.37) obtemos

$$
\begin{aligned}
& G\left(\mathbb{1}_{[b]}, x \mid \lambda\right) \geq \lambda^{-N} G\left(L_{\phi}^{N} \mathbb{1}_{[b]}, x \mid \lambda\right) \\
& =\lambda^{-N} \sum_{n \geq 0}^{\infty} \lambda^{-n} \sum_{\sigma^{n+N} y=x} e^{\phi_{n+N}(y)} \mathbb{1}_{[b]}(y) \\
& =\lambda^{-N} \sum_{n \geq 0}^{\infty} \lambda^{-n} \sum_{\begin{array}{c}
y_{1}, y_{2}, \ldots, y_{n+N-1} \in S \\
A\left(b, y_{1}\right)=\ldots=A\left(y_{n+N-1}, x_{0}\right)=1
\end{array}} e^{\phi_{n+N}\left(b y_{1} y_{2} \ldots y_{n+N-1} x\right)} \\
& \geq \lambda^{-N} \sum_{n \geq 0}^{\infty} \lambda^{-n} \sum_{\begin{array}{c}
y_{N+1}, y_{N+2}, \ldots, y_{n+N-1} \in S \\
A\left(a, y_{N+1}\right)=\ldots=A\left(y_{n+N-1}, x_{0}\right)=1
\end{array}} e^{\phi_{n+N}\left(b \xi_{1} \ldots \xi_{N-1} a y_{N+1} \ldots y_{n+N-1} x\right)} \\
& \geq \lambda^{-N} e^{\phi_{N}\left(b \xi_{1} \xi_{2} \ldots \xi_{N-1} x_{a}\right)-M_{1}} \\
& \sum_{n \geq 0}^{\infty} \lambda^{-n} \sum_{\substack{y_{N+1}, y_{N+2}, \ldots, y_{n+N-1} \in S \\
A\left(a, y_{N+1}\right)=\ldots=A\left(y_{n+N-1}, x_{0}\right)=1}} e^{\phi_{n}\left(a y_{N+1} \ldots y_{n+N-1} x\right)} \\
& =\lambda^{-N} e^{\phi_{N}\left(b \xi_{1} \xi_{2} \ldots \xi_{N-1} x_{a}\right)-M_{1}} G\left(\mathbb{1}_{[a]}, x \mid \lambda\right) .
\end{aligned}
$$

Definindo

$$
C_{a, b}:=\lambda^{N} e^{-\phi_{N}\left(b \xi_{1} \ldots \xi_{N-1} x_{a}\right)+M_{1}}
$$

e escolhendo $c_{a, b}=C_{b, a}^{-1}$ obtemos a Desigualdade (1.36).

Proposição 43. Sejam $\Sigma_{A}$ um shift transitivo localmente compacto e $\phi: \Sigma_{A} \rightarrow \mathbb{R}$ um potencial $\lambda$-transiente satisfazendo a condição de Walters. Então para cada $f \in C_{c}^{+}\left(\Sigma_{A}\right), f \neq 0$, existem $c_{f}>0$ e $C_{f}>0$ tais que

$$
c_{f} \leq K(f, x \mid \lambda) \leq C_{f}, \quad \text { para todo } \quad x \in \Sigma_{A} .
$$


Demonstração. Seja $f \in C_{c}^{+}\left(\Sigma_{A}\right)$. Como $\operatorname{supp}(f)$ é compacto, existem $N \in \mathbb{N}$ e $b_{1}, b_{2}, \ldots, b_{N} \in S$ tais que $\operatorname{supp}(f) \subset \cup_{i=1}^{N}\left[b_{i}\right]$. Logo

$$
f(x) \leq \sum_{i=1}^{N} \max _{\alpha \in\left[b_{i}\right]} f(\alpha) \mathbb{1}_{\left[b_{i}\right]}(x) \quad \text { para cada } x \in \Sigma_{A} .
$$

Sejam $o \in S$ um estado arbitrário e $x \in \Sigma_{A}$. Usando a Desigualdade (1.39) e o Lema 42 temos

$$
\begin{aligned}
K(f, x \mid \lambda) & =\frac{G(f, x \mid \lambda)}{G\left(\mathbb{1}_{[o]}, x \mid \lambda\right)} \\
& \leq \sum_{i=1}^{N} \frac{\max _{\alpha \in\left[b_{i}\right]} f(\alpha) G\left(\mathbb{1}_{\left[b_{i}\right]}, x \mid \lambda\right)}{G\left(\mathbb{1}_{[o]}, x \mid \lambda\right)} \\
& \leq \sum_{i=1}^{N} C_{b_{i}, o} \max _{\alpha \in\left[b_{i}\right]} f(\alpha) .
\end{aligned}
$$

Pelo fato que $f \neq 0$ é possível escolher $\left(\xi_{1} \xi_{2} \ldots \xi_{M}\right)$ uma palavra de tamanho $M \in \mathbb{N}$ tal que $c:=\min _{\alpha \in\left[\xi_{1} \xi_{2} \ldots \xi_{M}\right]} f(\alpha)>0$. Fixemos $x_{\xi_{M}} \in \sigma\left[\xi_{M}\right]$, usando o Lema 42 obtemos

$$
\begin{aligned}
G(f, x \mid \lambda) & \geq c G\left(\mathbb{1}_{\left[\xi_{1} \xi_{2} \ldots \xi_{M}\right]}, x \mid \lambda\right) \\
& \geq c \sum_{n=M-1}^{\infty} \lambda^{-n} L_{\phi}^{n} \mathbb{1}_{\left[\xi_{1} \xi_{2} \ldots \xi_{M}\right]}(x) \\
& =c \lambda^{-M+1} \sum_{n=0}^{\infty} \lambda^{-n} L_{\phi}^{n+M-1} \mathbb{1}_{\left[\xi_{1} \xi_{2} \ldots \xi_{M}\right]}(x) \\
& \geq c \lambda^{-M+1} e^{\phi_{M-1}\left(\xi_{1} \ldots \xi_{M} x_{\xi_{M}}\right)-\operatorname{Var} \phi} \sum_{n=0}^{\infty} \lambda^{-n} L_{\phi}^{n} \mathbb{1}_{\left[\xi_{M}\right]}(x) \\
& =c \lambda^{-M+1} e^{\phi_{M-1}\left(\xi_{1} \ldots \xi_{M} x_{\xi_{M}}\right)-\operatorname{Var} \phi} G\left(\mathbb{1}_{\left[\xi_{M}\right]}, x \mid \lambda\right) \\
& \geq c_{\xi_{M}, o} c \lambda^{-M+1} e^{\phi_{M-1}\left(\xi_{1} \ldots \xi_{M} x_{\xi_{M}}\right)-\operatorname{Var} \phi} G\left(\mathbb{1}_{[o]}, x \mid \lambda\right) .
\end{aligned}
$$

De (1.40) e (1.41) obtemos (1.38).

Para cada $f \in C_{c}^{+}\left(\Sigma_{A}\right)$ definimos

$$
C_{f}:=\sum_{i=1}^{N} C_{b_{i}, o} \max _{\alpha \in\left[b_{i}\right]} f(\alpha),
$$

onde $b_{1}, b_{2}, \ldots, b_{N} \in S$ tal que $\operatorname{supp}(f) \subset \cup_{i=1}^{N}\left[b_{i}\right]$.

Consideremos $\mathcal{W}=\left\{\omega_{i}\right\}_{i \geq 1}$ o conjunto de todas as palavras finitas. Definimos uma nova métrica $\rho$ em $\Sigma_{A}$, dada por

$$
\rho(x, y \mid \lambda)=\sum_{i=1}^{\infty} \frac{\left|K\left(\mathbb{1}_{\left[\underline{\left.\omega_{i}\right]}\right]}, x \mid \lambda\right)-K\left(\mathbb{1}_{\left[\underline{\omega}_{i}\right]}, y \mid \lambda\right)\right|+\left|\mathbb{1}_{\left[\underline{\left.\omega_{i}\right]}\right.}(x)-\mathbb{1}_{\left[\underline{\underline{\omega_{i}}}\right]}(y)\right|}{2^{i}\left(C_{\mathbb{1}_{\underline{\left.\omega_{i}\right]}}}+1\right)}
$$

pode-se mostrar que $\rho$ é uma métrica em $\Sigma_{A}$ e $\rho(x, y) \leq 3$ para todo $x, y \in \Sigma_{A}$.

Definição 44. Sejam $\Sigma_{A}$ um shift transitivo localmente compacto e $\phi: \Sigma_{A} \rightarrow \mathbb{R}$ um potencial $\lambda$-transiente satisfazendo a condição de Walters. A $\lambda$-compactificação de Martin para $\Sigma_{A}$ e $\phi$ é o completamento de $\Sigma_{A}$ pela métrica $\rho$ e esta é denotada por $\widehat{\Sigma_{A}}(\lambda)$. O $\lambda$-bordo de Martin para $\Sigma_{A}$ e фé $\mathcal{M}(\lambda):=\widehat{\Sigma_{A}}(\lambda) \backslash \Sigma_{A}$. 
A seguinte proposição descreve propriedades importantes do espaço métrico $\left(\widehat{\Sigma_{A}}, \rho\right)$.

Proposição 45. Sejam $\Sigma_{A}$ um shift transitivo localmente compacto e $\phi: \Sigma_{A} \rightarrow \mathbb{R}$ um potencial $\lambda$-transiente satisfazendo a condição de Walters. Logo:

1.) $\widehat{\Sigma_{A}}(\lambda)$ é $\rho$-compacto e $\mathcal{M}(\lambda)$ é $\rho$-fechado.

2.) Sejam $\left\{x_{n}\right\}_{n \geq 1} \subset \Sigma_{A}$ e $x \in \Sigma_{A}$. Então $x_{n} \stackrel{d}{\rightarrow} x$ se, e somente, se $x_{n} \stackrel{\rho}{\rightarrow} x$.

3.) Seja $A \subset \Sigma_{A}$. Então $A$ é $d$-aberto se, e somente, se $A$ é $\rho$-aberto.

4.) Sejam $\left\{x_{n}\right\}_{n \geq 1} \subset \Sigma_{A}$ e $\omega \in \mathcal{M}(\lambda)$ tais que $x_{n} \stackrel{\rho}{\rightarrow} \omega$. Então cada d-compacto contem no máximo uma quantidade finita de elementos $\left\{x_{n}\right\}_{n \geq 1}$, isto é, $x_{n}$ escapa de cada d-compacto.

5.) Para cada $f \in C_{c}\left(\Sigma_{A}\right), K(f, \cdot \mid \lambda)$ pode ser estendido de forma única para uma função contínua em $\widehat{\Sigma_{A}}(\lambda)$.

Demonstração. A seguir mostraremos somente os itens 4.) e 5.). Suponhamos que existe $A \subset \Sigma_{A}$ $d$-compacto tal que uma quantidade infinita de termos em $\left\{x_{n}\right\}_{n \geq 1}$ pertencem a $A$. Logo existe uma subsequencia convergente $\left\{x_{n_{k}}\right\}_{k \geq 1}$ tal que $x_{n_{k}} \stackrel{d}{\rightarrow} x \in A$. Pelo item 2.) temos que $x_{n_{k}} \stackrel{\rho}{\rightarrow} x \in A$ mas $x_{n} \rightarrow \omega \notin A$, isto é uma contradição e assim está mostrado o item 4.).

Sejam $f \in C_{c}^{+}\left(\Sigma_{A}\right)$ e $\omega \in \widehat{\Sigma_{A}}$. Escolhamos uma sequência arbitrária $\left\{x_{n}\right\}_{n \geq 1} \subset \Sigma_{A}$ tal que $x_{n} \stackrel{\rho}{\rightarrow} \omega$. Mostraremos que

$$
K(f, \omega \mid \lambda)=\lim _{x_{n} \stackrel{\rho}{\rightarrow}} K\left(f, x_{n} \mid \lambda\right)
$$

Primeiro mostraremos que $\left\{K\left(\mathbb{1}_{\left[\underline{\omega}_{i}\right]}, x_{n} \mid \lambda\right)\right\}_{n \geq 1}$ é uma sequencia de Cauchy para cada $\underline{\omega}_{i} \in \mathcal{W}$. Em efeito, fixado $\underline{\omega_{i}} \in \mathcal{W}$ existe $N\left(\underline{\omega_{i}}\right)>0$ tal que para todo $n, m>N\left(\underline{\omega_{i}}\right)$

$$
\frac{\left\|K\left(\mathbb{1}_{\left[\underline{\underline{\left.\omega_{i}\right]}}\right]}, x_{n} \mid \lambda\right)-K\left(\mathbb{1}_{\left[\underline{\left.\omega_{i}\right]}\right]}, x_{m} \mid \lambda\right)\right\|_{\infty}}{2^{i}\left(C_{\mathbb{1}_{\left[\underline{\left.\omega_{i}\right]}\right]}}+1\right)} \leq \rho\left(x_{n}, x_{m}\right)<\frac{\varepsilon}{3.2^{i}\left(C_{\mathbb{1}_{\left[\underline{\left.\omega_{i}\right]}\right.}}+1\right)} .
$$

$\operatorname{Logo}\left\{K\left(\mathbb{1}_{\left[\underline{\omega}_{i}\right]}, x_{n} \mid \lambda\right)\right\}_{n \geq 1}$ é uma sequencia de Cauchy. Desde que $0 \leq K\left(\mathbb{1}_{\left[\underline{\omega}_{i}\right]}, \cdot \mid \lambda\right) \leq C_{\mathbb{1}_{\omega_{i}}}$ temos que $\left\{K\left(\mathbb{1}_{\left[\omega_{i}\right]}, x_{n} \mid \lambda\right)\right\}_{n \geq 1}$ é convergente. Assim $K\left(\mathbb{1}_{\left[\underline{\omega}_{i}\right]}, \cdot \mid \lambda\right)$ pode-se estender de forma única e contínua em $\widehat{\Sigma_{A}}$, isto é,

$$
K\left(\mathbb{1}_{\left.\underline{\underline{\omega_{i}}}\right]}, \omega \mid \lambda\right)=\lim _{x_{n} \stackrel{\rho}{\rightarrow} \omega} K\left(\mathbb{1}_{\left[\underline{\omega_{i}}\right]}, x_{n} \mid \lambda\right) .
$$

Como o $\operatorname{supp}(f)$ é compacto existem $a_{1}, a_{2}, \ldots, a_{N} \in S$ tal que $\operatorname{supp}(f) \subset \cup_{i=1}^{N}\left[a_{i}\right]$. Além disso, como $\left\langle\mathbb{1}_{[\omega]}\right\rangle_{\underline{\omega} \in \mathcal{W}}$ é um subconjunto denso de $C_{c}\left(\Sigma_{A}\right)$ em relação a norma $\|\cdot\|_{\infty}$, dado $\varepsilon>0$ existem $\underline{\omega_{1}}, \underline{\omega_{2}}, \ldots, \underline{\omega_{M}} \in \mathcal{W}$ e $\alpha_{1}, \alpha_{2}, \ldots, \alpha_{M} \in \mathbb{R}^{+}$tais que

$$
\left\|f-\sum_{i=1}^{M} \alpha_{i} \mathbb{1}_{\underline{\underline{\omega}} \underline{\underline{\omega}}]}\right\|_{\infty}<\varepsilon / 3
$$

Escolhamos $C:=C(f) \geq \sum_{i=1}^{N} C_{\mathbb{1}_{\left[a_{i}\right]}}$. Para cada $x \in \Sigma_{A}$, usando a Desigualdade (1.44) e o fato que $\cup_{i=1}^{M}\left[\underline{\omega_{i}}\right] \subset \cup_{i=1}^{N}\left[a_{i}\right]$ obtemos

$$
\left\|K(f, x \mid \lambda)-K\left(\sum_{i=1}^{M} \alpha_{i} \mathbb{1}_{\left[\underline{\omega_{i}}\right]}, x \mid \lambda\right)\right\|_{\infty} \leq \frac{\varepsilon}{3}\left\|K\left(\sum_{i=1}^{N} \mathbb{1}_{\left[a_{i}\right]}, x \mid \lambda\right)\right\|_{\infty}
$$




$$
\begin{aligned}
& \leq \frac{\varepsilon}{3} \sum_{i=1}^{N}\left\|K\left(\mathbb{1}_{\left[a_{i}\right]}, x \mid \lambda\right)\right\|_{\infty} \\
& \leq \frac{\varepsilon}{3} \sum_{i=1}^{N} C_{\mathbb{1}_{\left[a_{i}\right]}} \\
& \leq \frac{\varepsilon}{3} C .
\end{aligned}
$$

Para todo $n, m>\left\{N\left(\underline{\omega_{1}}\right), N\left(\underline{\omega_{2}}\right), \ldots, N\left(\underline{\omega_{M}}\right)\right\}$ temos

$$
\begin{aligned}
& \left|K\left(f, x_{n} \mid \lambda\right)-K\left(f, x_{m} \mid \lambda\right)\right| \leq\left|K\left(f, x_{n} \mid \lambda\right)-K\left(\sum_{i=1}^{M} \alpha_{i} \mathbb{1}_{\left[\underline{\omega_{i}}\right]}, x_{n} \mid \lambda\right)\right| \\
& +\left|K\left(\sum_{i=1}^{M} \alpha_{i} \mathbb{1}_{\left[\underline{\left.\omega_{i}\right]}\right.}, x_{n} \mid \lambda\right)-K\left(\sum_{i=1}^{M} \alpha_{i} \mathbb{1}_{\left[\underline{\left.\omega_{i}\right]}\right.}, x_{m} \mid \lambda\right)\right| \\
& +\left|K\left(f, x_{m} \mid \lambda\right)-K\left(\sum_{i=1}^{M} \alpha_{i} \mathbb{1}_{\left[\underline{\omega_{i}}\right]}, x_{m} \mid \lambda\right)\right| \\
& \leq \frac{2 \varepsilon}{3} C+\left|K\left(\sum_{i=1}^{M} \alpha_{i} \underline{\mathbb{1}}_{\left.\underline{\underline{\omega_{i}}}\right]}, x_{n} \mid \lambda\right)-K\left(\sum_{i=1}^{M} \alpha_{i} \mathbb{1}_{\left[\underline{\underline{\omega_{i}}}\right]}, x_{m} \mid \lambda\right)\right| \\
& \leq \frac{2 \varepsilon}{3} C+\sum_{i=1}^{M}\left|\alpha_{i}\right|\left|K\left(\mathbb{1}_{\left[\underline{\omega_{i}}\right]}, x_{n} \mid \lambda\right)-K\left(\underline{\mathbb{1}}_{\left.\underline{\omega_{i}}\right]}, x_{m} \mid \lambda\right)\right| \\
& \leq \frac{2 \varepsilon}{3} C+\frac{\varepsilon}{3} \sum_{i=1}^{M}\left|\alpha_{i}\right| \\
& =\frac{\varepsilon}{3}\left(2 C+\sum_{i=1}^{M}\left|\alpha_{i}\right|\right) \text {. }
\end{aligned}
$$

De (1.47) temos que $\left\{K\left(f, x_{n} \mid \lambda\right)\right\}_{n \geq 1}$ é uma sequência de Cauchy de números reais, assim o limite $\lim _{n \rightarrow \infty} K\left(f, x_{n} \mid \lambda\right)$ existe e não depende da sequência $\left\{x_{n}\right\}_{n \geq 1}$. Assim obtemos (1.42).

Teorema 46 ([Cyr1], $\left.[\mathrm{Sw}]^{3}\right)$. Sejam $\Sigma_{A}$ um shift transitivo localmente compacto e $\phi: \Sigma_{A} \rightarrow \mathbb{R}$ um potencial $\lambda$-transiente com variação somável. Então existe uma medida $\sigma$-finita $\nu$ em $\Sigma_{A}$ tais que é positiva, finita em cada cilindro e $L_{\phi}^{*} \nu=\lambda \nu$, isto é,

$$
\int L_{\phi} f \mathrm{~d} \nu=\lambda \int f \mathrm{~d} \nu, \quad \text { para cada } f \in C_{c}\left(\Sigma_{A}\right) .
$$

Além disso existe uma função contínua $h: \Sigma_{A} \rightarrow(0,+\infty)$ tais que $L_{\phi} h=\lambda h$ e $h \mathrm{~d} \nu$ é uma medida finita $\sigma$-invariante.

Demonstração. Pelo item 5.) da Proposição 45, para cada $\omega \in M(\lambda)$ e $f \in C_{c}\left(\Sigma_{A}\right)$ temos que

$$
K(f, \omega \mid \lambda)=\lim _{x \rightarrow \omega} K(f, x \mid \lambda),
$$

é um funcional lineal contínua e positiva. Assim usando o Teorema de Representação de Riesz para espaços localmente compacto, para cada $\omega \in M(\lambda)$ existe uma medida $\nu_{\omega}$ finita em compactos tal que

$$
K(f, \omega \mid \lambda)=\int f \mathrm{~d} \nu_{\omega}, \quad \text { para cada } f \in C_{c}\left(\Sigma_{A}\right) .
$$

\footnotetext{
${ }^{3}$ V. Cyr mostrou a existência da automedida em [Cyr1] e O. Shwartz mostrou a existência da autofunção $h$ em $[\mathrm{Sw}]$.
} 
Pelo item 4.) da Proposição 45, para cada $f \in C_{c}\left(\Sigma_{A}\right)$ temos

$$
\begin{aligned}
\int L_{\phi} f \mathrm{~d} \nu_{\omega} & =K\left(L_{\phi} f, \omega \mid \lambda\right) \\
& =\lim _{x \stackrel{\rho}{\rho}_{\omega}} K\left(L_{\phi} f, x \mid \lambda\right) \\
& =\lambda \lim _{x \stackrel{\rho}{\rightarrow} \omega}\left(\frac{\sum_{n \geq 0} \lambda^{-n} L_{\phi}^{n} f(x)}{G\left(\mathbb{1}_{[o]}, x \mid \lambda\right)}-\frac{f(x)}{G\left(\mathbb{1}_{[o]}, x \mid \lambda\right)}\right) \\
& =\lambda \lim _{x \stackrel{\rho}{\rightarrow} \omega} K(f, x \mid \lambda)-\lambda \lim _{x \rightarrow \omega} \frac{f(x)}{G\left(\mathbb{1}_{[o]}, x \mid \lambda\right)} \\
& =\lambda K(f, \omega \mid \lambda) \\
& =\lambda \int f \mathrm{~d} \nu_{\omega} .
\end{aligned}
$$

Definindo

$$
h(x):=\sum_{i=1}^{\infty} \frac{1}{2^{n} C_{\mathbb{1}_{\left[a_{n}\right]}}} \mathbb{1}_{\left[a_{n}\right]}(x),
$$

temos que $h$ é uma função positiva e contínua tal que $L_{\phi} h=\lambda h$ e $h \mathrm{~d} \nu_{\omega}$ é uma medida finita e $\sigma$-invariante para cada $\omega \in \mathcal{M}(\lambda)$.

\subsection{Medidas de Equilíbrio}

Seja $(\Omega, \mathcal{F}, \mu)$ um espaço de probabilidade e $\beta$ uma partição mensurável finita ou enumerável de $\Omega$. A entropia da partição $\beta$ é

$$
H_{\mu}(\beta)=-\sum_{B \in \beta} \mu(B) \log \mu(B)
$$

onde consideramos $0 \log 0=0$.

Dadas duas partições $\alpha$ e $\beta$ de $\Omega$, definimos uma nova partição da seguinte maneira $\alpha \vee \beta:=$ $\{B \cap C \mid B \in \alpha, C \in \beta\}$. Seja $T: \Omega \rightarrow \Omega$ uma aplicação preservando a medida $\mu$, definimos $T^{-n} \beta:=\left\{T^{-n}(B) \mid B \in \beta\right\}$ e

$$
\beta^{n}:=\beta \vee T^{-1} \beta \vee \ldots \vee T^{-n+1} \beta
$$

Dada a partição $\beta$ definimos a entropia da aplicação $T$ por:

$$
h_{\mu}(T, \beta):=\lim _{n \rightarrow \infty} \frac{1}{n} H_{\mu}\left(\beta^{n}\right),
$$

o limite acima existe pelo Lema de Fakete.

Definição 47 (Entropia Métrica). Sejam $(\Omega, \mathcal{F}, \mu)$ um espaço de probabilidade e $T: \Sigma_{A} \rightarrow \Sigma_{A}$ uma aplicação preservando a medida, definimos a entropia métrica de $\mu$ como

$$
h_{\mu}(T):=\sup \left\{h_{\mu}(T, \beta)\right\}
$$

onde o supremo é tomado sobre todas as partições $\beta$ tal que $H_{\mu}(\beta)<\infty$.

Teorema 48 (Formula de Rokhlin). Sejam $\left(\Sigma_{A}, \sigma\right)$ shift de Markov e $\alpha:=\{[a] \mid a \in S\}$. Para cada medida de probabilidade $\sigma$-invariante $\mu$ temos:

i.) Se $H_{\mu}(\alpha)<\infty$, então $h_{\mu}(\sigma)=-\int \log \frac{\mathrm{d} \mu}{\mathrm{d} \mu \circ \sigma} \mathrm{d} \mu$. 
ii.) Se $H_{\mu}(\alpha)=\infty$, então $h_{\mu}(\sigma) \geq-\int \log \frac{\mathrm{d} \mu}{\mathrm{d} \mu \circ \sigma} \mathrm{d} \mu$.

Definição 49. Sejam $(\Omega, \mathcal{F}, \mu)$ um espaço de probabilidade, $\beta$ uma partição mensurável finita ou enumerável e $\mathcal{G} \subset \mathcal{F}$ uma sub- $\sigma$-álgebra. A função de informação de $\beta$ dado $\mathcal{G}$ é

$$
I_{\mu}(\beta \mid \mathcal{G}):=-\sum_{B \in \beta} \mathbb{1}_{B}(x) \log \mu(B \mid \mathcal{G})(x),
$$

onde $\mu(B \mid \mathcal{G})(x):=\mathbb{E}_{\mu}\left(\mathbb{1}_{B} \mid \mathcal{G}\right)(x)$.

Proposição 50 (Formula de Ledrappier). Sejam $\Sigma_{A}$ um shift de Markov, $\mathcal{B}$ a $\sigma$-álgebra gerada pelos cilindros e $\alpha:=\{[a] \mid a \in S\}$. Para cada medida de probabilidade $\sigma$-invariante $\mu$ temos

$$
I_{\mu}\left(\alpha \mid \sigma^{-1} \mathcal{B}\right)=-\log \frac{\mathrm{d} \mu}{\mathrm{d} \mu \circ \sigma} .
$$

O seguinte teorema chamado de Principio variacional foi provado para alfabetos finitos, shifts de Markov compactos, por D. Ruelle [Ru73] (ver também [Wal20]). O. Sarig [Sa5] provou para shifts de Markov com alfabeto enumerável e cardinalidade infinita e potenciais com variação somável e Y. Daon [Da] estendeu para potenciais que satisfazem a condição de Walters.

Teorema 51 (Principio Variacional). Sejam $\Sigma_{A}$ um shift topologicamente mixing e $\phi$ um potencial que satisfaz a condição de Walters tal que $\sup \phi<\infty$, então

$$
P_{G}(\phi)=\sup \left\{h_{\nu}(\sigma)+\int \phi \mathrm{d} \nu\right\}
$$

onde o supremo é tomado sobre todas as medidas de probabilidade $\sigma$-invariante $\nu$ tal que $-\int \phi d \nu<$ $\infty$.

A pressão de Gurevich para o potencial $\phi: \Sigma_{A} \rightarrow \mathbb{R}$ também pode ser obtida olhando as pressões de Gurevich do potencial restrita a subconjuntos compactos de $\Sigma_{A}$, veja o Teorema 53 .

Definição 52. Seja $\Sigma_{A}$ um shift de Markov com alfabeto $S$ e matriz de transição A. Um sub-sistema de $\Sigma_{A}$ é um shift de Markov com alfabeto $S^{\prime} \subset S$ e matriz de transição $A^{\prime}: S^{\prime} \times S^{\prime} \rightarrow\{0,1\}$ tal que se $A^{\prime}(i, j)=1$ então $A(i, j)=1$.

Teorema 53 (O. Sarig,[Sa1]). Sejam $\Sigma_{A}$ um shift topologicamente mixing e $\phi$ um potencial que satisfaz a condição de Walters, então

$$
P_{G}(\phi)=\sup \left\{P_{G}\left(\left.\phi\right|_{Y}\right) \mid Y \text { é um shift compacto topologicamente mixing sub-sistema de } \Sigma_{A}\right\} .
$$

Definição 54. Dado $\Sigma_{A}$ um shift de Markov e $\phi: \Sigma_{A} \rightarrow \mathbb{R}$ um potencial, a medida $\mu \in \mathcal{M}_{\sigma}^{1}\left(\Sigma_{A}\right)$ é chamada de equilíbrio para $\phi$ se

$$
h_{\mu}(\sigma)+\int \phi \mathrm{d} \mu=\sup \left\{h_{\nu}(\sigma)+\int \phi \mathrm{d} \nu\right\}
$$

onde o supremo é tomado sobre todas as medidas de probabilidade $\sigma$-invariante $\nu$ tal que $h_{\nu}(\sigma)+$ $\int \phi \mathrm{d} \nu$ está bem definido, ou seja é diferente de $-\infty$ ou $+\infty$.

Teorema 55 (Existência da Medida de Equilíbrio [Sa5].). Sob as hipoteses do Teorema 36 consideremos $m$ a medida de RPF do potencial $\phi: \Sigma_{A} \rightarrow \mathbb{R}$ tal que $\sup \phi<\infty$. Se $m$ tem entropia finita então $m$ é uma medida de equilíbrio para $\phi$. 
Demonstração. Denotemos $I_{m}:=I_{m}\left(\alpha \mid \sigma^{-1} \mathcal{B}\right)$ logo pela formula de Ledrappier, Proposição 50, temos que $I_{m}=-\log \frac{\mathrm{d} m}{\mathrm{~d} m \circ \sigma}$. Como $m=h \nu$ e $\frac{\mathrm{d} \nu}{\mathrm{d} \nu \circ \sigma}=\lambda^{-1} e^{\phi}$ obtemos

$$
I_{m}=-\left(\phi+\log h-\log h \circ \sigma-P_{G}(\phi)\right) .
$$

A seguir mostraremos duas afirmações que serão importantes para a prova do teorema:

Afirmação 1. $I_{m}, \phi+\log h-\log h \circ \sigma-P_{G}(\phi), \phi \in L^{1}(m)$

Por definição temos que $I_{m}$ é não negativa e pela formula de Rokhlin, Teorema 48, temos $\int I_{m} \mathrm{~d} m \leq h_{m}(\sigma)<\infty$, assim $I_{m} \in L^{1}(m)$. Logo por (1.49) temos que $\phi+\log h-\log h \circ \sigma-P_{G}(\phi) \in$ $L^{1}(m)$. É sabido que a medida de RPF $m$ é ergódica, veja [Sa5] Teorema 4.7, logo

i) Como $\sup \phi<\infty$, a convergência pontual do Teorema ergódico de Birkhoff vale para todas as funções integráveis unilaterais, logo

$$
\frac{\phi_{n}(x)}{n} \stackrel{n \rightarrow \infty}{\longrightarrow} \int \phi \mathrm{d} m, \quad m-\text { q.t.p.. }
$$

ii) Como $\phi+\log h-\log h \circ \sigma-P_{G}(\phi) \in L^{1}(m)$, então pelo Teorema ergódico de Birkhoff temos

$$
\frac{\phi_{n}(x)+\log h(x)-\log h\left(\sigma^{n} x\right)}{n} \stackrel{n \rightarrow \infty}{\longrightarrow} \int(\phi+\log h-\log h \circ \sigma) \mathrm{d} m, \quad m-\text { q.t.p. }
$$

iii) Pelo Teorema de recorrência de Poincaré e a continuidade de $h$ temos que existe $\left\{n_{k}(x)\right\} \uparrow \infty$ tal que

$$
\left|\log h(x)-\log h \circ \sigma^{n_{k}(x)}(x)\right|<1 .
$$

Dos itens $i$ ), $i$ ) e $i i i)$ temos que para cada $x \in \Sigma_{A} m$ - q.t.p.

$$
\begin{aligned}
\int \phi \mathrm{d} m & =\lim _{k \rightarrow \infty} \frac{1}{n_{k}(x)} \phi_{n_{k}(x)}(x) \\
& =\lim _{k \rightarrow \infty} \frac{1}{n_{k}(x)}\left(\phi_{n_{k}(x)}(x)+\log h(x)-\log h \circ \sigma^{n_{k}(x)}(x)\right) \\
& =\lim _{n \rightarrow \infty} \frac{1}{n}\left(\phi_{n}(x)+\log h(x)-\log h \circ \sigma^{n}(x)\right) \\
& =\int(\phi+\log h-\log h \circ \sigma) \mathrm{d} m .
\end{aligned}
$$

Assim $\int \phi \mathrm{d} m \neq-\infty$ pois $\int\left(\phi+\log h-\log h \circ \sigma-P_{G}(\phi)\right) \mathrm{d} m \neq-\infty$. Como $\sup \phi<\infty$ temos que $\phi \in L^{1}(m)$.

Afirmação 2. $\int(\log h-\log h \circ \sigma) \mathrm{d} m=0$.

Como $\phi,\left(\phi+\log h-\log h \circ \sigma-P_{G}(\phi)\right) \in L^{1}(m)$ então $(\log h-\log h \circ \sigma) \in L^{1}(m)$, mas

$$
\int \phi \mathrm{d} m=\int(\phi+\log h-\log h \circ \sigma) \mathrm{d} m
$$

$\operatorname{Logo} \int(\log h-\log h \circ \sigma) \mathrm{d} m=0$. 
Das Afirmações 1 e 2 obtemos:

$$
\begin{aligned}
h_{m}(\sigma)+\int \phi \mathrm{d} m & \geq \int I_{m} \mathrm{~d} m+\int \phi \mathrm{d} m \\
& =\int\left(I_{m}+\phi\right) \mathrm{d} m=\int\left(\log h \circ \sigma-\log h+P_{G}(\phi)\right) \mathrm{d} m=P_{G}(\phi) .
\end{aligned}
$$

A unicidade da medida de equilíbrio para espaços compactos foi provado por R. Bowen em [Bo74]. Para o caso de shifts de Markov não-compactos foi provado por O. Sarig e J. Buzzi em [BS] para potenciais com variação somável esse resultado foi estendido para potenciais satisfazendo a condição de Walters por Y. Daon em [Da].

Teorema 56 (Unicidade das medidas de equilíbrio, [Da]). Sejam $\Sigma_{A}$ shift topologicamente mixing e $\phi: \Sigma_{A} \rightarrow \mathbb{R}$ um potencial que satisfaz a condição de Walters tal que $\sup \phi<\infty$ e $P_{G}(\phi)<\infty$. Então:

i) ф tem no máximo uma medida de equilíbrio.

ii) Esta medida de equilíbrio, se existe, é igual a medida RPF de $\phi$.

iii) Em particular, se $\phi$ tem medida de equilíbrio então $\phi$ é positivamente recorrente e a medida de RPF tem entropia finita.

Notemos que este teorema diz que a medida de equilíbrio vem do operador de Ruelle. O Teorema 56 generaliza o Teorema demonstrado em [BS] o qual foi mostrado para potencias com variação somável.

Teorema 57 ([Sa5]). Sejam $\Sigma_{A}$ shift topologicamente mixing e $\phi, \psi: \Sigma_{A} \rightarrow \mathbb{R}$ dois potenciais satisfazendo a condição de Walters tais que $P_{G}(\phi), P_{G}(\psi), \sup \phi, \sup \psi<\infty$. Então $\phi \sim \psi+c$ se, e somente, se $\mu_{\phi}=\mu_{\psi}$. Onde $\mu_{\phi}$ e $\mu_{\psi}$ são as medidas de equilíbrio para os potenciais $\phi$ e $\psi$ respectivamente. 


\section{Capítulo 2}

\section{Medidas DLR}

$\mathrm{Na}$ área de formalismo termodinâmico o conceito de medida DLR foi introduzido por Dobrushin, Landford e Ruelle, e este é amplamente estudado assim como suas equivalências em relação as medidas conformes, de equilíbrio e o limite termodinâmico, veja [CiLo], [Mu1] e [Sa5].

As medidas DLR foram introduzidas como medidas de probabilidade. É conhecido que quando o shift de Markov topologicamente mixing tem alfabeto finito e o potencial satisfaz a condição de Walters, neste caso as medidas conformes são de probabilidade (Teorema de Ruelle-PerronFrobenius), além do mais, estas são equivalentes as medidas DLR, Teorema 91. Quando o alfabeto é enumerável com cardinalidade infinita o Teorema Generalizado de Ruelle-Perron-Frobenius mostra que as medidas conformes não são necessariamente de probabilidade, estas são $\sigma$-finitas podendo dar massa infinita ao espaço shift de Markov, veja o Exemplo 80. O fato de existirem medidas conformes $\sigma$-finitas inspira a estender a noção de medida DLR ao caso $\sigma$-finito e estudar as relações com as medidas conformes. Na primeira seção deste subseção vamos estudar o conceito de esperança condicional para medidas $\sigma$-finitas e na segunda subseção as relações entre medidas DLR e conformes no caso $\sigma$-finito.

\subsection{Medidas DLR finitas}

Suponhamos que $\Sigma_{A}$ seja um shift de Markov com alfabeto infinito enumerável. Para cada $k \geq 0$ as funções coordenadas $\pi_{k}: \Sigma_{A} \rightarrow \mathbb{R}$ são dadas por $\pi_{k}(x)=x_{k}$. A $\sigma$-álgebra de Borel $\mathcal{B}$ em $\Sigma_{A}$ é a $\sigma$-álgebra gerada pelos cilindros, e também é a menor $\sigma$-álgebra em relação a qual as funções coordenadas $\pi_{k}, k \geq 0$, são mensuráveis. Para cada $n \in \mathbb{N}$ denotamos $\sigma^{-n} \mathcal{B}$ como a menor $\sigma$-álgebra em relação a qual todas as funções coordenadas $\pi_{k}, k \geq n$, são mensuráveis. Assim obtemos uma família de $\sigma$-álgebras em $\Sigma_{A}$ satisfazendo

$$
\mathcal{B} \supset \sigma^{-1} \mathcal{B} \supset \sigma^{-2} \mathcal{B} \supset \ldots \supset \sigma^{-n} \mathcal{B} \supset \ldots
$$

Seja $\phi: \Sigma_{A} \rightarrow \mathbb{R}$ um potencial mensurável. Para cada $n \in \mathbb{N}$ definimos a seguinte aplicação $K_{n}: \mathcal{B} \times \Sigma_{A} \rightarrow[0,1]$, dada por

$$
K_{n}(E, x):=\frac{\sum_{\sigma^{n} y=\sigma^{n} x} e^{\phi_{n}(y)} \mathbb{1}_{E}(y)}{\sum_{\sigma^{n} y=\sigma^{n} x} e^{\phi_{n}(y)}}=\frac{L_{\phi}^{n}\left(\mathbb{1}_{E}\right)\left(\sigma^{n}(x)\right)}{L_{\phi}^{n}(\mathbb{1})\left(\sigma^{n}(x)\right)},
$$

onde $x \in \Sigma_{A}$, e $E \in \mathcal{B}$.

Observação 58. Notemos que em (2.1) o fator $\sum_{\sigma^{n} y=\sigma^{n} x} e^{\phi_{n}(y)}$ pode divergir para algum $n \in \mathbb{N}$ ou $x \in \Sigma_{A}$, logo deve ser considerada alguma regularidade no potencial e/ou no shift de Markov 
para que cada $K_{n}$ esteja bem definido.

Suponhamos que $\Sigma_{A}$ é o full shift com alfabeto finito, portanto um espaço compacto, e $\phi$ : $\Sigma_{A} \rightarrow \mathbb{R}$ um potencial contínuo. Neste caso $\sum_{\sigma^{n} y=\sigma^{n} x} e^{\phi_{n}(y)}<\infty$, para cada $n \in \mathbb{N}$ e $x \in \Sigma_{A}$. Além disso a aplicação $K_{n}(E, \cdot)$ é mensurável para cada $E \in \mathcal{B}$ e $n \in \mathbb{N}$, pois o operador de Ruelle está bem definido para potenciais contínuos num full shift com alfabeto finito.

Definição 59. Sejam $\Sigma_{A}$ um espaço shift de Markov e $\phi: \Sigma_{A} \rightarrow \mathbb{R}$ um potencial mensurável. Dizemos que uma família de aplicações $\left\{F_{n}\right\}_{n \geq 1}, F_{n}: \mathcal{B} \times \Sigma_{A} \rightarrow[0,1]$, é uma especificação Gibbsiana se para cada $n \in \mathbb{N}$ temos:

i.) $\mathcal{B} \ni E \mapsto F_{n}(E, x)$ é uma medida de probabilidade para cada $x \in \Sigma_{A}$;

ii.) $\Sigma_{A} \ni x \mapsto F_{n}(E, x)$ é $\sigma^{-n} \mathcal{B}-$ mensurável para cada $E \in \mathcal{B}$;

iii.) para cada $r \in \mathbb{N}$ e $f: \Sigma_{A} \rightarrow \mathbb{R}$ função limitada e $\mathcal{B}$-mensurável temos

$$
F_{n+r}(f, x)=F_{n+r}\left(F_{n}(f, \cdot), x\right), \quad \text { para cada } x \in \Sigma_{A} .
$$

Considerando $\Sigma_{A}$ o full shift com alfabeto finito e $\phi \in C\left(\Sigma_{A}\right)$, temos que $\left\{K_{n}\right\}_{n \geq 1}$ é uma especificação Gibbsiana, veja [CiLo]. Quando o alfabeto é infinito enumerável, o shift de Markov não é necessariamente compacto, neste caso pode se considerar alguma regularidade no potencial mensurável $\phi$ e/ou no shift de Markov tal que cada $K_{n}$ está bem definido, por exemplo:

1. Considerando $\Sigma_{A}$ um shift transitivo e $\phi: \Sigma_{A} \rightarrow \mathbb{R}$ um potencial localmente Hölder e somável. Nestas condições temos que o Operador de Ruelle está bem definido e $\sum_{\sigma^{n} y=\sigma^{n} x} e^{\phi_{n}(y)}<\infty$ para cada $n \in \mathbb{N}$ e $x \in \Sigma_{A}$, veja [MaUr1].

2. Seja $\Sigma_{A}$ um shift topologicamente mixing satisfazendo a condição $B I P$ e $\phi: \Sigma_{A} \rightarrow \mathbb{R}$ um potencial tais que $\sum_{n \geq 1} \operatorname{Var}_{n} \phi<\infty$ e $P_{G}(\phi)<\infty$. Neste caso o operador de Ruelle está bem definido e $\sum_{\sigma^{n} y=\sigma^{n} x} e^{\phi_{n}(y)}<\infty$ para cada $n \in \mathbb{N}$ e $x \in \Sigma_{A}$, veja [Sa5].

Deste ponto em diante consideraremos potenciais compativeis com cada $K_{n}$, isto é, potenciais tais que o Operador de Ruelle está bem definido e $\sum_{\sigma^{n} y=\sigma^{n} x} e^{\phi_{n}(y)}<\infty$ para cada $n \in \mathbb{N}$ e $x \in \Sigma_{A}$, isto é uma hipótese muito usual quando o conjunto de estados não é finito, veja o Capítulo 2 de [Ge].

Proposição 60. Sejam $\Sigma_{A}$ um shift de Markov e $\phi: \Sigma_{A} \rightarrow \mathbb{R}$ um potencial mensurável. A família de aplicações $\left\{K_{n}\right\}_{n \geq 1}$ definidas em (2.1) é uma especificação Gibbsiana.

Demonstração. Notemos que a prova do item $i$.) da Definição 59 é trivial. Fixemos $n \geq 1$, lembremos que uma aplicação $z: \Sigma_{A} \rightarrow \mathbb{R}$ é $\sigma^{-n} \mathcal{B}$-mensurável se, e somente, se $z$ pode-se escrever como $z=v \circ \sigma^{n}$, onde $v$ é uma aplicação $\mathcal{B}$-mensurável. Dado $E \in \mathcal{B}$ definimos

$$
v(\xi):=\frac{\sum_{\sigma^{n} y=\xi} e^{\phi_{n}(y)} \mathbb{1}_{E}(y)}{\sum_{\sigma^{n} y=\xi} e^{\phi_{n}(y)}},
$$

notemos que $v$ é $\mathcal{B}$-mensurável pois $v(\xi)=\frac{\left(L_{\phi}^{n} \mathbb{1}_{E}\right)(\xi)}{\left(L_{\phi}^{n} \mathbb{1}\right)(\xi)}$ e por hipótese o operador de Ruelle está bem definido para cada $n \in \mathbb{N}$. Desde que $K_{n}(E, x)=v\left(\sigma^{n} x\right)$ para todo $x \in \Sigma_{A}$ então $K_{n}(\cdot, x) \mapsto \mathbb{R}$ é $\sigma^{-n} \mathcal{B}$-mensurável. Assim obtemos o item ii.) da Definição 59. 
A seguir mostraremos o item $i i i$.). Fixemos $n \geq 1$, pelo item $i$.) temos que

$$
K_{n}(E, x)=\int_{E} d K_{n}(\cdot, x)=\int \mathbb{1}_{E}(y) d K_{n}(y, x),
$$

para todo $x \in \Sigma_{A}$ e $E \in \mathcal{B}$. Seja $s: \Sigma_{A} \rightarrow \mathbb{R}$ uma função simples, da Equação (2.2) obtemos

$$
K_{n}(s, x)=\int s(y) d K_{n}(y, x) .
$$

Seja $\varphi: \Sigma_{A} \rightarrow[0, \infty)$ uma função mensurável e limitada, logo existem uma família $\left\{s_{m}\right\}_{m \geq 1}$ de funções simples tal que $0 \leq s_{1} \leq s_{2} \leq \ldots \leq s_{m} \leq \ldots \leq \varphi$ e $s_{m} \stackrel{m \rightarrow \infty}{\longrightarrow} \varphi$, usando a Equação (2.3) e o Teorema da Convergência Monótona obtemos

$$
\begin{aligned}
\int_{\Sigma_{A}} \varphi d K_{n}(\cdot, x) & =\lim _{m \rightarrow \infty} \int_{\Sigma_{A}} s_{m} d K_{n}(\cdot, x) \\
& =\lim _{m \rightarrow \infty} K_{n}\left(s_{m}, x\right) \\
& =\lim _{m \rightarrow \infty} \frac{\sum_{\sigma^{n} y=\sigma^{n} x} e^{\phi_{n}(y)} s_{m}(y)}{\sum_{\sigma^{n} y=\sigma^{n} x} e^{\phi_{n}(y)}} \\
& =\frac{\sum_{\sigma^{n} y=\sigma^{n} x} e^{\phi_{n}(y)} \varphi(y)}{\sum_{\sigma^{n} y=\sigma^{n} x} e^{\phi_{n}(y)}} .
\end{aligned}
$$

Desde que $K_{n}(\cdot, x)$ é uma medida para cada $x \in \Sigma_{A}$ então (2.4) pode ser escrito como

$$
K_{n}(\varphi, x)=\frac{\sum_{\sigma^{n} y=\sigma^{n} x} e^{\phi_{n}(y)} \varphi(y)}{\sum_{\sigma^{n} y=\sigma^{n} x} e^{\phi_{n}(y)}} .
$$

No caso em que $\varphi$ toma valores reais basta repetir argumento apresentado acima para $\varphi^{+}$e $\varphi^{-}$. A. Lopes e L. Cioletti em [CiLo] mostram o item iii.) da Definição 59 para o espaço full shift de Markov com alfabeto finito, esta prova também é válida para o espaço shift de Markov com alfabeto infinito enumerável.

Definição 61 (Medidas $\phi$-DLR). Sejam $\Sigma_{A}$ um shift de Markov e $\phi: \Sigma_{A} \rightarrow \mathbb{R}$ um potencial mensurável. Dizemos que $\mu \in \mathcal{M}^{1}\left(\Sigma_{A}\right)$ é uma medida $\phi-\mathrm{DLR}$ se para todo $n \in \mathbb{N}$ e para toda função $\varphi: \Sigma_{A} \rightarrow \mathbb{R}$ mensurável e limitada temos

$$
\mathbb{E}_{\mu}\left[\varphi \mid \sigma^{-n} \mathcal{B}\right](x)=K_{n}(\varphi, x), \quad \mu-\text { q.t.p. } .
$$

Pela próxima proposição temos que a Equação (2.5) só precisa ser verificada para funções características suportadas em cilindros.

Proposição 62. Sejam $\Sigma_{A}$ um shift de Markov, $\phi: \Sigma_{A} \rightarrow \mathbb{R}$ um potencial mensurável e $\mu \in$ $\mathcal{M}^{1}\left(\Sigma_{A}\right)$. Logo $\mu$ é $\phi-\mathrm{DLR}$ se, e somente, se para cada $n \in \mathbb{N} e[\underline{a}]$ cilindro de tamanho $n$ temos

$$
\mathbb{E}_{\mu}\left[\mathbb{1}_{[a]} \mid \sigma^{-n} \mathcal{B}\right](x)=K_{n}\left(\mathbb{1}_{[\underline{a}]}, x\right), \quad \mu-\text { q.t.p. }
$$

Demonstração. Sejam $\mu$ uma medida $\phi-$ DLR e $n \in \mathbb{N}$. Para cada $\underline{a} \in \mathcal{W}_{n}$ pela Definição (61) obtemos (2.6). 
Agora mostraremos a recíproca. Para mostrar que $\mu$ é $\phi$-DLR basta provar que para todo $n \in \mathbb{N}$ e para todo cilindro $\left[a_{0}, a_{1}, \ldots, a_{m-1}\right]$ de tamanho $m \in \mathbb{N}$ temos

$$
\mathbb{E}_{\mu}\left[\mathbb{1}_{\left[a_{0}, \ldots, a_{m-1}\right]} \mid \sigma^{-n} \mathcal{B}\right](x)=\frac{L_{\phi}^{n} \mathbb{1}_{\left[a_{0}, \ldots, a_{m-1}\right]}\left(\sigma^{n} x\right)}{L_{\phi}^{n} \mathbb{1}\left(\sigma^{n} x\right)}, \quad \mu-\text { q.t.p. } .
$$

pois isso pode ser estendido para a álgebra gerada pelos cilindros. E o teorema da extensão de Caratheodory estende isso para a $\sigma$-álgebra de Borel completando a prova. Fixemos $n, m \in \mathbb{N}$

i.) Suponhamos que $m \leq n$. Seja $\left[a_{0}, \ldots, a_{m-1}\right]$ um cilindro arbitrário de tamanho $m$, logo pode-se escrever como a união enumerável disjunta de cilindros de tamanho $n$, isto é,

$$
\left[a_{0}, \ldots, a_{m-1}\right]=\cup_{i \geq 1} E_{i}
$$

onde cada $E_{i}$, para $i \geq 1$, é um cilindro de tamanho $n$ e $E_{i} \cap E_{j}=\emptyset$ quando $i \neq j$. Definindo $s_{k}(x)=\sum_{i=1}^{k} \mathbb{1}_{E_{i}}(x), x \in \Sigma_{A}$, temos que

$$
0 \leq s_{1} \leq s_{2} \leq \ldots \leq s_{k} \leq \ldots \leq \mathbb{1}_{\left[a_{0}, \ldots, a_{m-1}\right]} \quad \text { e } \quad s_{k} \stackrel{k \rightarrow \infty}{\longrightarrow} \mathbb{1}_{\left[a_{0}, \ldots, a_{m-1}\right]}
$$

$\operatorname{logo} K_{n}\left(s_{k}, x\right) \stackrel{k \rightarrow \infty}{\longrightarrow} K_{n}\left(\mathbb{1}_{\left[a_{0}, \ldots, a_{m-1}\right]}, x\right)$. Usando o Teorema da Convergência Monótona para esperança condicional obtemos

$$
\begin{aligned}
\mathbb{E}_{\mu}\left[\mathbb{1}_{\left[a_{0}, \ldots, a_{m-1}\right]} \mid \sigma^{-n} \mathcal{B}\right](x) & =\lim _{k \rightarrow \infty} \mathbb{E}_{\mu}\left[s_{k} \mid \sigma^{-n} \mathcal{B}\right](x) \\
& =\lim _{k \rightarrow \infty} \sum_{i=1}^{k} \mathbb{E}_{\mu}\left[\mathbb{1}_{E_{i}} \mid \sigma^{-n} \mathcal{B}\right](x) \\
& =\lim _{k \rightarrow \infty} \sum_{i=1}^{k} K_{n}\left(\mathbb{1}_{E_{i}}, x\right) \\
& =\lim _{k \rightarrow \infty} K_{n}\left(s_{k}, x\right) \\
& =K_{n}\left(\mathbb{1}_{\left[a_{0}, \ldots, a_{m-1}\right]}, x\right) \\
& =\frac{L_{\phi}^{n} \mathbb{1}_{\left[a_{0}, \ldots, a_{m-1}\right]}\left(\sigma^{n} x\right)}{\left.L_{\phi}^{n} \mathbb{1}^{n} x\right)}, \quad \mu-\text { q.t.p. }
\end{aligned}
$$

ii.) Suponhamos que $m>n$, fixemos $m=n+1$. Seja $\left[a_{0}, \ldots, a_{n}\right]$ um cilindro arbitrário, notemos que $\mathbb{1}_{\left[a_{0}, \ldots, a_{n}\right]}=\mathbb{1}_{\left[a_{0}, \ldots, a_{n-1}\right]} \cdot \mathbb{1}_{\left[a_{n}\right]} \circ \sigma^{n}$ e $\mathbb{1}_{\left[a_{n}\right]} \circ \sigma^{n}$ é uma função $\sigma^{-n} \mathcal{B}$-mensurável, logo

$$
\begin{aligned}
& \mathbb{E}_{\mu}\left[\mathbb{1}_{\left[a_{0}, \ldots, a_{n}\right]} \mid \sigma^{-n} \mathcal{B}\right](x)=\mathbb{1}_{\left[a_{n}\right]} \circ \sigma^{n}(x) \cdot \mathbb{E}_{\mu}\left[\mathbb{1}_{\left[a_{0}, \ldots, a_{n-1}\right]} \mid \sigma^{-n} \mathcal{B}\right](x) \\
&=\mathbb{1}_{\left[a_{n}\right]} \circ \sigma^{n}(x) K_{n}\left(\mathbb{1}_{\left[a_{0}, \ldots, a_{n-1}\right]}, x\right) \\
&=\mathbb{1}_{\left[a_{n}\right]} \circ \sigma^{n}(x) \cdot \frac{\sum_{\sigma^{n} \sigma^{n} x} e^{\phi_{n}(y)} \mathbb{1}_{\left[a_{0}, \ldots, a_{n-1}\right]}(y)}{\sum_{\sigma^{n} y=\sigma^{n} x} e^{\phi_{n}(y)}} \\
&=\frac{\sum_{\sigma^{n} y=\sigma^{n} x} e^{\phi_{n}(y)} \mathbb{1}_{\left[a_{0}, \ldots, a_{n-1}\right]}(y) \cdot \mathbb{1}_{\left[a_{n}\right]} \circ \sigma^{n}(y)}{\sum_{\sigma^{n} y=\sigma^{n} x} e^{\phi_{n}(y)}} \\
&=\frac{\sigma^{n} y=\sigma^{n} x}{e^{\phi_{n}(y)} \mathbb{1}_{\left[a_{0}, \ldots, a_{n-1}, a_{n}\right]}(y)} \\
& \sum_{\sigma^{n} y=\sigma^{n} x} e^{\phi_{n}(y)}
\end{aligned}
$$




$$
=\frac{L_{\phi}^{n} \mathbb{1}_{\left[a_{0}, \ldots, a_{n}\right]}\left(\sigma^{n} x\right)}{L_{\phi}^{n} \mathbb{1}\left(\sigma^{n} x\right)}, \quad \mu-\text { q.t.p. . }
$$

Dos itens i.) e ii.) obtemos a Equação (2.7).

Observação 63. Notemos que fixando $n \in \mathbb{N}$ e $x \in \Sigma_{A}$ o lado direito da Equação (2.7)

$$
\mu\left(\cdot, \sigma^{-n} \mathcal{B}\right)(x):=\mathbb{E}_{\mu}\left[\cdot \mid \sigma^{-n} \mathcal{B}\right](x),
$$

é bem comportada como uma medida e podemos fazer a extensão de uma álgebra para uma $\sigma$-álgebra. Isso é porque $\mu$ é uma medida condicional regular definida no espaço polonês $\Sigma_{A}$.

A proposição acima nos fornece uma maneira mais fácil de definir as medidas $\phi-D L R$, isto é, $\mu$ é $\phi-D L R$ se para cada $n \in \mathbb{N}$ e $\underline{a} \in \mathcal{W}_{n}$ temos

$$
\mathbb{E}_{\mu}\left[\mathbb{1}_{[\underline{a}]} \mid \sigma^{-n} \mathcal{B}\right](x)=\frac{e^{\phi_{n}\left(\underline{a} \sigma^{n} x\right)} \mathbb{1}_{\left\{\underline{a} \sigma^{n} x \in \Sigma_{A}\right\}}}{\sum_{\sigma^{n} y=\sigma^{n} x} e^{\phi_{n}(y)}}, \quad \mu-\text { q.t.p. . }
$$

As Equações (2.8) são chamadas de DLR. A seguir temos uma proposição que caracteriza as medidas DLR, para os detalhes da prova veja a Proposição 2.1 em [Sa5].

Proposição 64. Sejam $\Sigma_{A}$ um shift de Markov, $\mu \in \mathcal{M}^{1}\left(\Sigma_{A}\right)$ e $\phi: \Sigma_{A} \rightarrow \mathbb{R}$ um potencial mensurável. Então $\mu$ é $\phi-\mathrm{DLR}$ se, e somente, se para cada par de cilindros $[\underline{a}]:=\left[a_{0}, a_{1}, \ldots, a_{n-1}\right]$ $e[\underline{b}]:=\left[b_{0}, b_{1}, \ldots, b_{n-1}\right]$ de tamanho $n \in \mathbb{N}$ tais que $a_{n-1}=b_{n-1}$ e $\mu([\underline{a}])>0$, a aplicação $v_{\underline{a}, \underline{b}}:[\underline{a}] \rightarrow[\underline{b}]$ definida por $v_{\underline{a}, \underline{b}}:\left(\underline{a} x_{n}^{\infty}\right) \mapsto\left(\underline{b}_{n}^{\infty}\right)$ satisfaz

$$
\frac{\mathrm{d} \mu \circ v_{\underline{a}, \underline{b}}}{\mathrm{~d} \mu}=e^{\phi_{n}\left(\underline{b} x_{n}^{\infty}\right)-\phi_{n}\left(\underline{a}_{n}^{\infty}\right)}, \quad \mu-\text { q.t.p. } \operatorname{em} \mathcal{B} \cap[\underline{a}] .
$$

A seguir temos dois corolários que possuem informações sobre a medida de cilindros quando esta é DLR.

Corolário 65. Sejam $\Sigma_{A}$ um shift de Markov, $\phi: \Sigma_{A} \rightarrow \mathbb{R}$ um potencial mensurável e $\mu$ uma medida $\phi-$ DLR. Fixamos $n \in \mathbb{N}$ e $c \in S$.

i.) Se existe $\underline{w} \in \mathcal{W}_{n}(c)$ tal que $\mu([\underline{w}])=0$, então $\forall \underline{\omega} \in \mathcal{W}_{n}(c)$ temos $\mu([\underline{\omega}])=0$.

ii.) Se para $\underline{w} \in \mathcal{W}_{n}(c)$ tal que $\mu([\underline{w}])>0$, então $\forall \underline{\omega} \in \mathcal{W}_{n}(c)$ temos $\mu([\underline{\omega}])>0$.

Demonstração. A prova é uma consequência direta da Proposição 64.

Corolário 66. Sejam $\Sigma_{A}$ um shift de Markov topologicamente mixing satisfazendo a propriedade BIP e $\phi: \Sigma_{A} \rightarrow \mathbb{R}$ um potencial mensurável. Se $\mu$ é uma medida $\phi-\mathrm{DLR}$ então $\mu([a])>0$, para todo $a \in S$.

Demonstração. Como $\Sigma_{A}$ é topologicamente mixing e satisfaz a propriedade $B I P$, temos que $\Sigma_{A}$ é finitamente primitivo. Seja $k_{0} \in \mathbb{N}$ como no item $i v$.) da Definição 2. Pelo fato de $\mu$ ser uma medida de probabilidade, existe $a \in S$ tal que $\mu([a])>0$. Podemos escrever o cilindro $[a]$ como a união disjunta de cilindros de tamanho $k_{0}+2$. Como $\mu([a])>0$, existe um cilindro $[a, \ldots, c] \subset[a]$ de tamanho $k_{0}+2$ tal que $\mu([a, \ldots, c])>0$.

Vamos denotar $\underline{\xi}:=(a, \ldots, c)$. Fixemos $b \in S$, logo pelo fato de $\Sigma_{A}$ ser finitamente primitivo, 
temos que existe uma palavra $w(b, c)$ de tamanho $k_{0}$ tal que $[b, w(b, c), c] \neq \emptyset$ e denotemos $\underline{\eta}:=$ $(b, w(b, c), c)$. Pela Proposição 64, temos

$$
\mu \circ v_{\underline{\xi}, \underline{\eta}}(E)=\int_{E} e^{\phi_{k_{0}+2}\left(\underline{\eta} x_{k_{0}+2}^{\infty}\right)-\phi_{k_{0}+2}\left(\underline{\xi} x_{k_{0}+2}^{\infty}\right)} \mathrm{d} \mu(x),
$$

para todo $E \in \mathcal{B} \cap[\underline{\xi}]$. Substituindo $E=[\underline{\xi}]$ na Equação $(2.10)$ temos $\mu([\underline{\eta}])>0 \operatorname{logo} \mu([b])>0$. Como $b \in S$ foi arbitrário, temos $\mu([b])>0$ para todo $b \in S$.

A seguir mostraremos uma série de resultados para o espaço full shift.

Corolário 67. Sejam $\Sigma_{A}$ o full shift de alfabeto $\mathbb{N}, \mu \in \mathcal{M}^{1}\left(\Sigma_{A}\right)$ e $\phi: \Sigma_{A} \rightarrow \mathbb{R}$ um potencial mensurável. Se $\mu$ é $\phi-\mathrm{DLR}$ então

i.) $\mu([a])>0$, para todo $a \in \mathbb{N}$.

ii.) Fixados $a, b \in \mathbb{N}$. Seja $\widetilde{v}_{a b}:[a] \rightarrow[b]$ a aplicação definida por, $\widetilde{v}_{a b}:\left(a x_{1}^{\infty}\right) \rightarrow\left(b x_{1}^{\infty}\right)$, logo

$$
\left.\frac{\mathrm{d} \mu \circ \widetilde{v}_{a b}}{\mathrm{~d} \mu}\right|_{[a]}=e^{\phi\left(b x_{1}^{\infty}\right)-\phi\left(a x_{1}^{\infty}\right)}, \quad \mu-\text { q.t.p. em } \mathcal{B} \cap[a] .
$$

iii.) O potencial $\phi$ não pode ser constante.

Demonstração. Como todo full shift é $B I P$, pelo Corolário 66 , temos $\mu([a])>0$, para todo $a \in \mathbb{N}$. Sejam $a, b \in \mathbb{N}$ e $\underline{c}$ uma palavra de tamanho arbitrário, logo:

$$
\begin{aligned}
& \mu \circ \widetilde{v}_{a b}([a \underline{c}])=\mu([b \underline{c}])=\int_{[b c]} \mathrm{d} \mu=\int_{\Sigma_{A}} \mathbb{1}_{[b c]} \mathrm{d} \mu \\
& =\int_{\Sigma_{A}} \mathbb{1}_{[c]} \circ \sigma(x) \mathbb{1}_{[b]}(x) \mathrm{d} \mu(x) \\
& =\int_{\Sigma_{A}} \mathbb{1}_{[\underline{c}]} \circ \sigma(x) \mathbb{E}\left(\mathbb{1}_{[b]} \mid \sigma^{-1} \mathcal{B}\right)(x) \mathrm{d} \mu(x) \\
& =\int_{\Sigma_{A}} \mathbb{1}_{[\underline{c}]} \circ \sigma(x) \frac{e^{\phi\left(b x_{1}^{\infty}\right)}}{\sum_{\sigma y=\sigma x} e^{\phi(y)}} \mathrm{d} \mu(x) \\
& =\int_{\Sigma_{A}} \mathbb{1}_{[\underline{c}]} \circ \sigma(x) e^{\phi\left(b x_{1}^{\infty}\right)-\phi\left(a x_{1}^{\infty}\right)} \frac{e^{\phi\left(a x_{1}^{\infty}\right)}}{\sum_{\sigma y=\sigma x} e^{\phi(y)}} \mathrm{d} \mu(x) \\
& =\int_{\Sigma_{A}} \mathbb{1}_{[\underline{c}]} \circ \sigma(x) e^{\phi\left(b x_{1}^{\infty}\right)-\phi\left(a x_{1}^{\infty}\right)} \mathbb{E}\left(\mathbb{1}_{[a]} \mid \sigma^{-1} \mathcal{B}\right)(x) \mathrm{d} \mu(x) \\
& =\int_{\Sigma_{A}} \mathbb{1}_{[\underline{c}]} \circ \sigma(x) e^{\phi\left(b x_{1}^{\infty}\right)-\phi\left(a x_{1}^{\infty}\right)} \mathbb{1}_{[a]}(x) \mathrm{d} \mu(x) \\
& =\int_{\Sigma_{A}} \mathbb{1}_{[a c]}(x) e^{\phi\left(b x_{1}^{\infty}\right)-\phi\left(a x_{1}^{\infty}\right)} \mathrm{d} \mu(x) \\
& =\int_{[a \underline{c}]} e^{\phi\left(b x_{1}^{\infty}\right)-\phi\left(a x_{1}^{\infty}\right)} \mathrm{d} \mu(x) .
\end{aligned}
$$

Desde que $\left\{[a \underline{c}] \mid \underline{c} \in \mathcal{W}_{n}\right.$ para $\left.n \geq 1\right\}$ é uma base da topologia induzida em $[a] \cap \mathcal{B}$ e $\mu$ é uma medida regular obtemos que

$$
\mu \circ \widetilde{v}_{a b}(E)=\int_{E} e^{\phi\left(b x_{1}^{\infty}\right)-\phi\left(a x_{1}^{\infty}\right)} \mathrm{d} \mu(x)
$$

para cada $E \in \mathcal{B} \cap[a]$. Assim obtemos o item ii.). Seja $\phi=$ cte e $a \in S$ fixado, logo pela Equação (2.11) temos que $\mu([b])=\mu([a])$ para todo $b \in \mathbb{N}$, isto contradiz o fato de $\mu$ ser uma medida de probabilidade. 
O seguinte teorema dado por O. Sarig mostra que neste caso todas as medidas conformes de probabilidade são DLR. Para os detalhes da prova, veja a Proposição 2.2 em [Sa5].

Teorema 68. Sejam $\Sigma_{A}$ um shift de Markov e $\phi: \Sigma_{A} \rightarrow \mathbb{R}$ um potencial mensurável. Se $m$ é uma medida de probabilidade $(\phi, \lambda)$-conforme e não-singular, para algum $\lambda>0$, então $m$ é $\phi-$ DLR.

\subsection{Medidas DLR $\sigma$-finitas}

Neste capítulo estenderemos a noção de medida DLR para o caso $\sigma$-finito.

\subsubsection{Esperança Condicional para Medidas $\sigma$-finitas}

Nesta subseção estenderemos a definição de esperança condicional para medidas $\sigma$-finitas, o resultado principal desta subseção é o Teorema da Convergência de Martingais para medidas $\sigma$-finitas.

Teorema 69 (Radon-Nikodým). Sejam $(\Omega, \mathcal{F})$ um espaço mensurável, $\nu$ e $\mu$ duas medidas $\sigma$-finitas definidas em $\mathcal{F}$. Se $\nu \ll \mu$ então existe uma função $\mathcal{F}$-mensurável $g: \Omega \rightarrow[0,+\infty]$ tal que

$$
\nu(E)=\int_{E} g \mathrm{~d} \mu, \quad \text { para todo } E \in \mathcal{F} .
$$

Além do mais, $g$ é unicamente determinada $\mu$ - q.t.p..

Teorema 70. Sejam $(\Omega, \mathcal{F}, \mu)$ um espaço de medida, $\mathcal{G}$ uma sub- $\sigma$-álgebra de $\mathcal{F}$ e $\nu:=\left.\mu\right|_{\mathcal{G}}$ uma medida $\sigma$-finita em $\mathcal{G}$. Então para cada $f \in L^{1}(\Omega, \mathcal{F}, \mu)$ existe uma função $g \in L^{1}(\Omega, \mathcal{G}, \nu)$ tal que

$$
\int_{E} f \mathrm{~d} \mu=\int_{E} g \mathrm{~d} \nu, \quad \text { para } \operatorname{cada} E \in \mathcal{G} .
$$

Além do mais se $g^{\prime} \in L^{1}(\Omega, \mathcal{G}, \nu)$ é uma outra função satisfazendo a igualdade acima, então $g=g^{\prime}$ $\nu$ - q.t.p..

Demonstração. Considere $f \geq 0$. Seja $\eta: \mathcal{F} \rightarrow[0,+\infty)$ a medida definida por

$$
\eta(E)=\int_{E} f \mathrm{~d} \mu .
$$

Notemos que para todo $E \in \mathcal{G}$ tal que $\mu(E)=0$ a integral acima é igual a zero $\operatorname{logo} \eta(E)=0$, e assim temos $\left.\left.\eta\right|_{\mathcal{G}} \ll \mu\right|_{\mathcal{G}}$, além disso $\left.\eta\right|_{\mathcal{G}}$ e $\left.\mu\right|_{\mathcal{G}}$ são $\sigma$-finitas. Daí segue do Teorema de RadonNikodým que existe uma função $\mathcal{G}$-mensurável $g: \Omega \rightarrow[0, \infty]$ tal que

$$
\eta(E)=\int_{E} g \mathrm{~d} \nu, \quad \text { para todo } E \in \mathcal{G} .
$$

Das Equações (2.13) e (2.14) obtemos a Equação (2.12) e tomando $E=\Omega$ temos que $g \in L^{1}(\Omega, \mathcal{G}, \nu)$. O Teorema de Radon-Nikodým garante que $g$ é unicamente determinada $\nu$ - q.t.p. e portanto o teorema fica provado no caso em que $f \geq 0$. No caso em que $f$ toma valores reais basta repetir o argumento apresentado acima para $f^{+}$e $f^{-}$.

O teorema acima nos permite definir a esperança condicional no caso de medidas $\sigma$-finitas, note que o preço a pagar é que a medida tem que ser $\sigma$-finita na sub- $\sigma$-álgebra restrita.

Definição 71 (Esperança Condicional). Sejam $(\Omega, \mathcal{F}, \mu)$ um espaço de medida, $f \in L^{1}(\Omega, \mathcal{F}, \mu)$, $\mathcal{G} \subset \mathcal{F}$ uma sub- $\sigma$-álgebra e $\nu:=\left.\mu\right|_{\mathcal{G}}$ uma medida $\sigma$-finita em $\mathcal{G}$. Dizemos que uma função 
$g \in L^{1}(\Omega, \mathcal{G}, \nu)$ é a esperança condicional de $f$ dada a $\sigma$-álgebra $\mathcal{G}$, se

$$
\int_{E} f \mathrm{~d} \mu=\int_{E} g \mathrm{~d} \nu, \quad \text { para todo } E \in \mathcal{G} .
$$

A esperança condicional é denotada por $g(x):=\mathbb{E}_{\mu}[f \mid \mathcal{G}](x)$.

Sejam $(\Omega, \mathcal{F}, \mu)$ um espaço de medida tal que $\mu(\Omega)=\infty$ e $\mathcal{G} \subset \mathcal{F}$ uma sub- $\sigma$-álgebra, dizemos que $\mathcal{G}$ é compatível com a esperança condicional se esta está bem definida, isto é, se $\mu$ é $\sigma$-finita em $\mathcal{G}$ e assim pelo Teorema 70 a esperança condicional pode ser definida. A seguir citamos algumas das propriedades da esperança condicional, estas são análogas ao caso das medidas de probabilidade.

Proposição 72 (Propriedades da Esperança Condicional). Sejam $(\Omega, \mathcal{F}, \mu)$ um espaço de medida $e \mathcal{G} \subset \mathcal{F}$ uma sub- $\sigma$-álgebra compativel com a esperança condicional. Logo

i.) Se $f \in L^{1}(\Omega, \mathcal{G}, \mu)$, então

$$
\mathbb{E}_{\mu}[f \mid \mathcal{G}]=f, \quad \mu-\text { q.t.p. }
$$

ii.) Se $f \in L^{1}(\Omega, \mathcal{F}, \mu)$ então

$$
\mathbb{E}_{\mu}\left[\mathbb{E}_{\mu}[f \mid \mathcal{F}] \mid \mathcal{G}\right]=\mathbb{E}_{\mu}[f \mid \mathcal{G}], \quad \mu-\text { q.t.p.. }
$$

iii.) Desigualdade de Jensen, se $f \in L^{1}(\Omega, \mathcal{F}, \mu)$ então

$$
\left|\mathbb{E}_{\mu}[f \mid \mathcal{F}]\right| \leq \mathbb{E}_{\mu}[|f| \mid \mathcal{F}], \quad \mu-\text { q.t.p.. }
$$

Daqui em diante, quando é entendido quem é a medida omitiremos esta da notação da esperança condicional, isto é, $\mathbb{E}[f \mid \mathcal{F}]:=\mathbb{E}_{\mu}[f \mid \mathcal{F}]$. No restante desta sub-seção vamos a provar uma série de resultados necessários para obter o Teorema da Convergência de Martingais, Teorema 76, análogo ao caso de probabilidade.

Lema 73. Sejam $(\Omega, \mathcal{F}, \mu)$ um espaço de medida e $\mathcal{F}_{1} \subset \mathcal{F}_{2} \subset \mathcal{F}_{3} \ldots \subset \mathcal{F}_{n} \subset \ldots$ sub- $\sigma$-álgebras de $\mathcal{F}$ cada uma delas compatível com a esperança condicional. Então para toda $f \in L^{1}(\Omega, \mathcal{F}, \mu) e$ $a>0$ temos

$$
\mu\left(E_{a}\right) \leq \frac{1}{a} \int|f| \mathrm{d} \mu,
$$

onde $E_{a}=\left\{x \in \Omega \mid \sup _{n} \mathbb{E}\left[f \mid \mathcal{F}_{n}\right](x)>a\right\}$.

Demonstração. Consideremos $f \geq 0$ e

$$
E_{a}^{n}=\left\{x \in \Omega \mid \mathbb{E}\left[f \mid \mathcal{F}_{n}\right](x)>a, \mathbb{E}\left[f \mid \mathcal{F}_{i}\right](x) \leq a \text { para } i=1,2, \ldots, n-1\right\},
$$

é fácil verificar que $E_{a}^{n} \in \mathcal{F}_{n}$ para cada $n \in \mathbb{N}$ e $E_{a}^{n} \cap E_{a}^{m}=\emptyset$ quando $n \neq m$, além do mais $E_{a}$ pode-se escrever como $E_{a}=\sqcup_{n \geq 1} E_{a}^{n}$.

Logo

$$
\begin{aligned}
\int_{E_{a}} f \mathrm{~d} \mu & =\sum_{n \geq 1} \int_{E_{a}^{n}} f \mathrm{~d} \mu=\sum_{n \geq 1} \int_{E_{a}^{n}} \mathbb{E}\left[f \mid \mathcal{F}_{n}\right] \mathrm{d} \mu \\
& \geq \sum_{n \geq 1} a \mu\left(E_{a}^{n}\right)=a \mu\left(E_{a}\right) .
\end{aligned}
$$

Portanto $\mu\left(E_{a}\right) \leq \frac{1}{a} \int f \mathrm{~d} \mu$. 
De maneira semelhante pode-se mostrar que

$$
\mu(|f|>a) \leq \frac{1}{a} \int|f| \mathrm{d} \mu,
$$

para toda $f \in L^{1}(\Omega, \mathcal{F}, \mu)$.

Teorema 74 (Aproximação de uma $\sigma$-álgebra por uma álgebra geradora, [KiTa]). Sejam $\mathcal{A}$ uma álgebra, $(\Omega, \mathcal{F}, \mu)$ um espaço de medida onde $\mu$ é não-negativa e $\mathcal{F}=\sigma(\mathcal{A})$. Se $\mu$ é $\sigma$-finita em $\mathcal{A}$ então para todo $B \in \mathcal{F}$ com $\mu(B)<\infty$ e para cada $\varepsilon>0$ existe $A \in \mathcal{A}$ tal que $\mu(B \triangle A)<\varepsilon$.

Do teorema acima é fácil ver que $\mu(A)<\infty$ e $\int_{\Omega}\left|\mathbb{1}_{B}-\mathbb{1}_{A}\right| \mathrm{d} \mu<\varepsilon$. Uma aplicação direta deste teorema é o seguinte lema que nos permitirá provar o Teorema da Convergência Martingais.

Lema 75. Sejam $(\Omega, \mathcal{F}, \mu)$ um espaço de medida e $\mathcal{F}_{1} \subset \mathcal{F}_{2} \subset \mathcal{F}_{3} \ldots \subset \mathcal{F}_{n} \subset \ldots$ sub- $\sigma$-álgebras de $\mathcal{F}$. Seja $\mathcal{F}_{\infty}:=\sigma\left(\cup_{n \geq 1} \mathcal{F}_{n}\right)$. Se $\mu$ é $\sigma-$ finita em $\cup_{n \geq 1} \mathcal{F}_{n}$ então $\cup_{n \geq 1} L^{1}\left(\Omega, \mathcal{F}_{n}, \mu\right)$ é denso em $L^{1}\left(\Omega, \mathcal{F}_{\infty}, \mu\right)$ em relação a norma $L^{1}$, isto é, para cada $f \in L^{1}\left(\Omega, \mathcal{F}_{\infty}, \mu\right)$ e cada $\varepsilon>0$ existem $m \in \mathbb{N}$ e $g \in L^{1}\left(\Omega, \mathcal{F}_{m}, \mu\right)$ tal que $\int_{\Omega}|f-g| \mathrm{d} \mu<\varepsilon$.

Demonstração. Seja $\mathbb{1}_{B} \in L^{1}\left(\Omega, \mathcal{F}_{\infty}, \mu\right)$ e $\varepsilon>0$, pelo Teorema 74 , existem $m \in \mathbb{N}$ e $A \in \mathcal{F}_{m}$ tal que $\mathbb{1}_{A} \in L^{1}\left(\Omega, \mathcal{F}_{m}, \mu\right)$ e $\int_{\Omega}\left|\mathbb{1}_{B}-\mathbb{1}_{A}\right| \mathrm{d} \mu<\varepsilon$, logo o lema é verdadeiro para funções características.

Consideremos a função simples $s=\sum_{i=1}^{k} c_{i} \mathbb{1}_{B_{i}} \in L^{1}\left(\Omega, \mathcal{F}_{\infty}, \mu\right)$ e $\varepsilon>0$, logo para cada $1 \leq i \leq k$ temos $B_{i} \in \mathcal{F}_{\infty}$ e $\mu\left(B_{i}\right)<\infty$. Pelo Teorema 74 temos que para cada $B_{i}$, com $1 \leq i \leq k$, existem $m_{i} \in \mathbb{N}$ e $A_{i} \in \mathcal{F}_{m_{i}}$ tal que

$$
\int_{\Omega}\left|\mathbb{1}_{B_{i}}-\mathbb{1}_{A_{i}}\right| \mathrm{d} \mu<\varepsilon /\left(\sum_{i=1}^{k}\left|c_{i}\right|\right) .
$$

Seja $\widetilde{s}:=\sum_{i=1}^{k} c_{i} \mathbb{1}_{A_{i}}$ temos que $\widetilde{s} \in L^{1}\left(\Omega, \mathcal{F}_{m}, \mu\right)$ onde $m:=\max \left\{m_{1}, m_{2}, \ldots, m_{k}\right\}$. Além disso pela Desigualdade (2.16) temos

$$
\int_{\Omega}|s-\widetilde{s}| \mathrm{d} \mu \leq \sum_{i=1}^{k}\left|c_{i}\right| \int_{\Omega}\left|\mathbb{1}_{B_{i}}-\mathbb{1}_{A_{i}}\right| \mathrm{d} \mu<\varepsilon .
$$

Assim temos que o lema é satisfeito para funções simples. Sejam $f \in L^{1}\left(\Omega, \mathcal{F}_{\infty}, \mu\right)$ tal que $f \geq 0$ e $\varepsilon>0$, desde que $f$ é $\mathcal{F}_{\infty}$-mensurável existem funções simples, $\mathcal{F}_{\infty}$-mensuráveis, não-decrescentes $0 \leq s_{1} \leq s_{2} \leq \ldots \leq s_{n} \leq \ldots \leq f$ tal que $\lim _{n \rightarrow \infty} s_{n}(x)=f(x)$ e $\lim _{n \rightarrow \infty} \int_{\Omega} s_{n} \mathrm{~d} \mu=\int_{\Omega} f \mathrm{~d} \mu$. Logo existe $n_{0} \in \mathbb{N}$ tal que $\int_{\Omega}\left(f-s_{n_{0}}\right) \mathrm{d} \mu<\varepsilon / 2$. Seja $\widetilde{s}_{n_{0}} \in L^{1}\left(\Omega, \mathcal{F}_{n_{0}}, \mu\right)$ a função simples correspondente a $s_{n_{0}}$ como no passo anterior tal que $\int_{\Omega}\left|s_{n_{0}}-\widetilde{s}_{n_{0}}\right| \mathrm{d} \mu \leq \varepsilon / 2$, logo

$$
\int_{\Omega}|f-\widetilde{s}| \mathrm{d} \mu \leq \int_{\Omega}\left|f-s_{n_{0}}\right| \mathrm{d} \mu+\int_{\Omega}\left|s_{n_{0}}-\widetilde{s}_{n_{0}}\right| \mathrm{d} \mu<\varepsilon .
$$

No caso em que $f \in L^{1}\left(\Omega, \mathcal{F}_{\infty}, \mu\right)$ toma valores reais basta repetir o argumento apresentado acima para $f^{+}$e $f^{-}$.

Teorema 76 (Teorema da Convergência de Martingais $\sigma$-finito). Sejam $(\Omega, \mathcal{F}, \mu)$ um espaço de medida e $\mathcal{F}_{1} \subset \mathcal{F}_{2} \subset \mathcal{F}_{3} \ldots \subset \mathcal{F}_{n} \subset \ldots$ sub- $\sigma$-álgebras de $\mathcal{F}$ cada uma delas compatível com a esperança condicional. Seja $\mathcal{F}_{\infty}:=\sigma\left(\cup_{n \geq 1} \mathcal{F}_{n}\right)$. Se $f \in L^{1}(\Omega, \mathcal{F}, \mu)$ e $\mathcal{F}_{\infty}$ é compatível com a esperança condicional então

$$
\mathbb{E}\left[f \mid \mathcal{F}_{n}\right] \stackrel{n \rightarrow \infty}{\longrightarrow} \mathbb{E}\left[f \mid \mathcal{F}_{\infty}\right], \quad \mu-\text { q.t.p. e em } L^{1}(\mu) .
$$

Demonstração. Seja $f \in L^{1}(\Omega, \mathcal{F}, \mu)$ e $\varepsilon>0$ logo pelo lema acima existem $m \in \mathbb{N}$ e $g \in$ $L^{1}\left(\Omega, \mathcal{F}_{m}, \mu\right)$ tal que $\left\|\mathbb{E}\left[f \mid \mathcal{F}_{\infty}\right]-g\right\|_{1}<\varepsilon$, assim pelo item ii.) da Proposição 72 para todo 
$n>m$ temos o seguinte

$$
\begin{aligned}
\left|\mathbb{E}\left[f \mid \mathcal{F}_{n}\right]-\mathbb{E}\left[f \mid \mathcal{F}_{\infty}\right]\right| & =\left|\mathbb{E}\left[\mathbb{E}\left[f \mid \mathcal{F}_{\infty}\right] \mid \mathcal{F}_{n}\right]-\mathbb{E}\left[f \mid \mathcal{F}_{\infty}\right]\right| \\
& =\left|\mathbb{E}\left[\mathbb{E}\left[f \mid \mathcal{F}_{\infty}\right]-g \mid \mathcal{F}_{n}\right]+g-\mathbb{E}\left[f \mid \mathcal{F}_{\infty}\right]\right| \\
& \leq \mathbb{E}\left[\mathbb{E}\left[f \mid \mathcal{F}_{\infty}\right]-g|| \mathcal{F}_{n}\right]+\left|\mathbb{E}\left[f \mid \mathcal{F}_{\infty}\right]-g\right|, \quad \mu \text { - q.t.p.. }
\end{aligned}
$$

Logo

$$
\begin{aligned}
\left\|\mathbb{E}\left[f \mid \mathcal{F}_{n}\right]-\mathbb{E}\left[f \mid \mathcal{F}_{\infty}\right]\right\|_{1} & \leq\left\|\mathbb{E}\left[\left|\mathbb{E}\left[f \mid \mathcal{F}_{\infty}\right]-g\right| \mid \mathcal{F}_{n}\right]\right\|_{1}+\left\|\mathbb{E}\left[f \mid \mathcal{F}_{\infty}\right]-g\right\|_{1} \\
& =\left\|\mathbb{E}\left[f \mid \mathcal{F}_{\infty}\right]-g\right\|_{1}+\left\|\mathbb{E}\left[f \mid \mathcal{F}_{\infty}\right]-g\right\|_{1}<2 \varepsilon
\end{aligned}
$$

Portanto

$$
\mathbb{E}\left[f \mid \mathcal{F}_{n}\right] \underset{n \rightarrow \infty}{\stackrel{L^{1}(\mu)}{\longrightarrow}} \mathbb{E}\left[f \mid \mathcal{F}_{\infty}\right], \quad \forall f \in L^{1}(\Omega, \mathcal{F}, \mu) .
$$

A seguir mostraremos a convergência $\mu$ - q.t.p.. Sejam $\tilde{f} \in L^{1}\left(\Omega, \mathcal{F}_{\infty}, \mu\right)$ e $\varepsilon>0$, pela definição da esperança condicional temos que $\mathbb{E}\left[\tilde{f} \mid \mathcal{F}_{\infty}\right]=\widetilde{f}, \mu$ - q.t.p., e pelo Lema 75 existem $n_{0} \in \mathbb{N}$ e $g \in L^{1}\left(\Omega, \mathcal{F}_{n_{0}}, \mu\right)$ tal que $\|\tilde{f}-g\|_{1}<\varepsilon / 2$. Para $n>n_{0}$

$$
\begin{aligned}
\left|\mathbb{E}\left[\tilde{f} \mid \mathcal{F}_{n}\right]-\tilde{f}\right| & \leq\left|\mathbb{E}\left[\tilde{f}-g \mid \mathcal{F}_{n}\right]-(\tilde{f}-g)\right| \\
& \leq\left|\mathbb{E}\left[\tilde{f}-g \mid \mathcal{F}_{n}\right]\right|+|\tilde{f}-g| \\
& \leq \mathbb{E}\left[|\widetilde{f}-g| \mid \mathcal{F}_{n}\right]+|\widetilde{f}-g|, \quad \text { pela desigualdade de Jensen. }
\end{aligned}
$$

Usando o Lema 73 e a Desigualdade (2.15) obtemos

$$
\begin{aligned}
\mu\left(\left\{x \in \Omega\left|\limsup _{n \rightarrow \infty}\right| \mathbb{E}\left[\tilde{f} \mid \mathcal{F}_{n}\right]-\widetilde{f} \mid>\sqrt{\varepsilon}\right\}\right) \leq & \mu\left(\left\{x \in \Omega \mid \limsup _{n \rightarrow \infty}\left(\mathbb{E}\left[|\tilde{f}-g| \mid \mathcal{F}_{n}\right]+|\tilde{f}-g|\right)>\sqrt{\varepsilon}\right\}\right) \\
\leq & \mu\left(\left\{x \in \Omega\left|\limsup _{n \rightarrow \infty} \mathbb{E}\left[|\tilde{f}-g| \mid \mathcal{F}_{n}\right]+\right| \tilde{f}-g \mid>\sqrt{\varepsilon}\right\}\right) \\
\leq & \mu\left(\left\{x \in \Omega \mid \limsup _{n \rightarrow \infty} \mathbb{E}\left[|\tilde{f}-g| \mid \mathcal{F}_{n}\right]>\frac{\sqrt{\varepsilon}}{2}\right\}\right) \\
& \quad+\mu\left(\left\{x \in \Omega|| \tilde{f}-g \mid>\frac{\sqrt{\varepsilon}}{2}\right\}\right) \\
\leq & \mu\left(\left\{x \in \Omega \mid \sup _{n} \mathbb{E}\left[|\widetilde{f}-g| \mid \mathcal{F}_{n}\right]>\frac{\sqrt{\varepsilon}}{2}\right\}\right) \\
& \quad+\mu\left(\left\{x \in \Omega|| \tilde{f}-g \mid>\frac{\sqrt{\varepsilon}}{2}\right\}\right) \\
\leq & \frac{2}{\sqrt{\varepsilon}} \int|\tilde{f}-g| \mathrm{d} \mu+\frac{2}{\sqrt{\varepsilon}} \int|\tilde{f}-g| \mathrm{d} \mu \\
\leq & 4 \sqrt{\varepsilon} .
\end{aligned}
$$

Desde que $\varepsilon$ é arbitrário

$$
\mu\left(\left\{x \in \Omega\left|\limsup _{n \rightarrow \infty}\right| \mathbb{E}\left[\widetilde{f} \mid \mathcal{F}_{n}\right]-\widetilde{f} \mid>\sqrt{\varepsilon}\right\}\right) \stackrel{\varepsilon \rightarrow 0^{+}}{\longrightarrow} 0
$$

é dizer $\lim \sup _{n \rightarrow \infty}\left|\mathbb{E}\left[\widetilde{f} \mid \mathcal{F}_{n}\right]-\widetilde{f}\right|=0$, $\mu$ - q.t.p.. Portanto

$$
\mathbb{E}\left[\widetilde{f} \mid \mathcal{F}_{n}\right] \underset{n \rightarrow \infty}{\stackrel{\mu-\text { q.t.p. }}{\longrightarrow}} \widetilde{f} .
$$


Seja $f \in L^{1}(\Omega, \mathcal{F}, \mu), \operatorname{logo} \mathbb{E}\left[f \mid \mathcal{F}_{\infty}\right] \in L^{1}\left(\Omega, \mathcal{F}_{\infty}, \mu\right)$. Substituindo $\mathbb{E}\left[f \mid \mathcal{F}_{\infty}\right]$ por $\tilde{f}$ em (2.19) e usando o item ii.) da Proposição 72 obtemos

$$
\mathbb{E}\left[f \mid \mathcal{F}_{n}\right] \stackrel{\mu-\text { q.t.p. }}{=} \mathbb{E}\left[\mathbb{E}\left[f \mid \mathcal{F}_{\infty}\right] \mid \mathcal{F}_{n}\right] \underset{n \rightarrow \infty}{\stackrel{\mu-\text { q.t.p. }}{\longrightarrow}} \mathbb{E}\left[f \mid \mathcal{F}_{\infty}\right] .
$$

\subsubsection{Medidas DLR e Medidas Conformes no caso $\sigma$-finito}

O Teorema Generalizado de Ruelle-Perron-Frobenius garante a existência de medidas conforme $\sigma$-finitas. Para definir as medidas DLR no caso em que a medida não for finita deveríamos garantir que cada esperança condicional $\left\{\mathbb{E}\left[\cdot \mid \sigma^{-n} \mathcal{B}\right]\right\}_{n \geq 1}$ esteja bem definida, isso pela Definição 71 é equivalente a pedir que cada sub- $\sigma$-álgebra

$$
\sigma^{-1} \mathcal{B} \supset \sigma^{-2} \mathcal{B} \supset \ldots \supset \sigma^{-n} \mathcal{B} \supset \ldots
$$

seja compatível com a esperança condicional. É assim que chegamos à seguinte definição de medida DLR para o caso de medidas não finitas.

Definição 77 (Medida DLR $\sigma$-finita). Sejam $\Sigma_{A}$ um shift de Markov, $\mu \in \mathcal{M}\left(\Sigma_{A}\right)$ e $\phi: \Sigma_{A} \rightarrow \mathbb{R}$ um potencial mensurável. Dizemos que $\mu$ é uma medida $\phi-\mathrm{DLR}$ se para cada $n \in \mathbb{N}$ temos que:

i.) A sub- $\sigma$-álgebra $\sigma^{-n} \mathcal{B}$ é compatível com a esperança condicional,

ii.) Para cada $\underline{a} \in \mathcal{W}_{n}$ temos

$$
\mathbb{E}_{\mu}\left(\mathbb{1}_{[\underline{a}]} \mid \sigma^{-n} \mathcal{B}\right)(x)=\frac{e^{\phi_{n}\left(\underline{a} \sigma^{n} x\right)} \mathbb{1}_{\left\{\underline{a} \sigma^{n} x \in \Sigma_{A}\right\}}}{\sum_{\sigma^{n} y=\sigma^{n} x} e^{\phi_{n}(y)}}, \quad \mu-\text { q.t.p.. }
$$

Notemos que quando $\mu$ é uma medida de probabilidade cada sub- $\sigma$-álgebra de $\left\{\sigma^{-n} \mathcal{B}\right\}_{n \geq 1}$ é compatível com a esperança condicional e neste caso a definição de DLR no caso $\sigma$-finito coincide com a definição tradicional.

A seguinte proposição mostra que impondo uma hipótese no potencial cada sub- $\sigma$-álgebra de $\left\{\sigma^{-n} \mathcal{B}\right\}_{n \geq 1}$ é compatível com a esperança condicional em relação a qualquer medida $\lambda$-conforme.

Proposição 78. Sejam $\Sigma_{A}$ um shift de Markov, $\phi: \Sigma_{A} \rightarrow \mathbb{R}$ um potencial mensurável e $\nu$ uma medida $(\phi, \lambda)$-conforme, para algum $\lambda>0$, tal que $\nu([a])<\infty$ para cada $a \in S$. Se $\left\|L_{\phi} \mathbb{1}\right\|_{\infty}<\infty$ então $\nu\left(\pi_{n}^{-1}\{a\}\right)<\infty$ para cada $n \in \mathbb{N}$ e $a \in S$. Em particular, cada $\sigma^{-n} \mathcal{B}$ é compatível com a esperança condicional para cada $n \geq 1$.

Demonstração. Fixemos $n \in \mathbb{N}$. Sejam $a \in S$ e $w_{0}, w_{1}, \ldots, w_{n-1}$ uma palavra de tamanho $n$ tal que $A\left(w_{n-1}, a\right)=1, \log 0$

$$
\begin{aligned}
\lambda^{n} \nu\left(\left[w_{0}, w_{1}, \ldots, w_{n-1}, a\right]\right) & =\int L_{\phi}^{n} \mathbb{1}_{\left[w_{0}, w_{1}, \ldots, w_{n-1}, a\right]}(x) \mathrm{d} \nu \\
& =\sum_{b \in S} \int_{[b]} \sum_{\sigma^{n} y=x} e^{\phi_{n}(y)} \mathbb{1}_{\left[w_{0}, w_{1}, \ldots, w_{n-1}, a\right]}(y) \mathrm{d} \nu(x) \\
& =\int_{[a]} \sum_{\sigma^{n} y=x} e^{\phi_{n}(y)} \mathbb{1}_{\left[w_{0}, w_{1}, \ldots, w_{n-1}, a\right]}(y) \mathrm{d} \nu(x) \\
& =\int_{[a]} e^{\phi_{n}\left(w_{0}, w_{1}, \ldots, w_{n-1}, x\right)} \mathrm{d} \nu(x) .
\end{aligned}
$$


Notemos que $\pi_{n}^{-1}\{a\}=\bigcup_{\substack{\left[w_{0}, w_{1}, \ldots, w_{n-1}\right] \neq \emptyset \\ A\left(w_{n-1}, a\right)=1}}\left[w_{0}, w_{1}, \ldots, w_{n-1}, a\right]$. Somando em $(2.21)$ sobre todos os cilindros que pertencem a $\overline{\mathcal{W}}_{n+1}(a)$, temos que

$$
\begin{aligned}
\nu\left(\pi_{n}^{-1}\{a\}\right) & =\lambda^{-n} \sum_{\substack{\left[w_{0}, w_{1}, \ldots, w_{n-1}\right] \neq \emptyset \\
A\left(w_{n-1}, a\right)=1}} \int_{[a]} e^{\phi_{n}\left(w_{0}, w_{1}, \ldots, w_{n-1}, x\right)} \mathrm{d} \nu(x) \\
& =\lambda^{-n} \int_{\substack{[a] \\
\left[w_{0}, w_{1}, \ldots, w_{n-1}\right] \neq \emptyset \\
A\left(w_{n-1}, a\right)=1}} e^{\phi_{n}\left(w_{0}, w_{1}, \ldots, w_{n-1}, x\right)} \mathrm{d} \nu(x) \\
& =\lambda^{-n} \int_{[a]} L_{\phi}^{n} \mathbb{1} \mathrm{d} \nu \\
& \leq \lambda^{-n} \nu([a])\left\|L_{\phi} \mathbb{1}\right\|_{\infty}^{n}<\infty .
\end{aligned}
$$

Logo $\nu\left(\pi_{n}^{-1}\{a\}\right)<\infty$ para cada $n \geq 1$ e $a \in S$. Portanto cada sub- $\sigma$-álgebra $\sigma^{-n} \mathcal{B}$ é compatível com a esperança condicional, para $n \geq 1$.

Observação 79. As medidas conformes associadas a potenciais satisfazendo $\left\|L_{\phi} \mathbb{1}\right\|_{\infty}<\infty$ não são necessariamente finitas, podem dar massa infinita ao espaço $\Sigma_{A}$. Veja o Exemplo 37.

Sarig exibiu um shift de Markov que não satisfaz a propriedade $B I P$, um potencial mensurável e uma medida $(\phi, 1)$-conforme tal que a autofunção não está afastada de zero, veja [Sa5]. A seguir mostramos que esta medida conforme falha a condição $\left\|L_{\phi} \mathbb{1}\right\|_{\infty}<\infty$ e existem cilindros generalizados com massa infinita.

Exemplo 80. Sejam $\Sigma_{A}$ o shift de Markov com alfabeto $\mathbb{N}$ dado pela seguinte matriz de transição

$$
A=\left[\begin{array}{llllll}
0 & 1 & 0 & 0 & 0 & \cdots \\
1 & 0 & 1 & 0 & 0 & \\
1 & 0 & 0 & 1 & 0 & \\
1 & 0 & 0 & 0 & 1 & \\
\vdots & & & & & \ddots
\end{array}\right],
$$

e o potencial $\phi: \Sigma_{A} \rightarrow \mathbb{R}$ definido por $\phi(x)=\log \left(p_{x_{1}} p_{x_{1} x_{2}} / p_{x_{2}}\right)$, onde

$$
p_{n}=\frac{1}{n} ; \quad p_{i j}=\left\{\begin{array}{cl}
1-\frac{1}{i}, & j=1, i>1 ; \\
\frac{1}{i}, & j=i+1 ; \\
0, & \text { caso contrário. }
\end{array}\right.
$$

Note que $\Sigma_{A}$ é topologicamente mixing e não satisfaz a propriedade BIP. O potencial tem variação somável, $\operatorname{Var}_{1} \phi<\infty$ e $\left\|L_{\phi} \mathbb{1}\right\|_{\infty}=+\infty$. A automedida do operador de Ruelle é dada por $\nu\left(\left[a_{0}, a_{1}, \cdots, a_{n}\right]\right)=p_{a_{0}} p_{a_{0} a_{1}} \cdots p_{a_{n-1} a_{n}}$.

Veja que $\nu([a])=\frac{1}{a}<\infty$ para cada $a \in \mathbb{N}$. No entanto $\nu\left(\pi_{1}^{-1}\{1\}\right)=+\infty$.

A seguir temos uma caracterização das medidas DLR, esta é análoga ao caso de probabilidade 
dada por O. Sarig, veja a Proposição 64.

Proposição 81. Sejam $\Sigma_{A}$ o espaço shift de Markov, $\phi: \Sigma_{A} \rightarrow \mathbb{R}$ um potencial mensurável e $\nu$ uma medida tais que $\nu\left(\pi_{n}^{-1}\{a\}\right)<\infty$ para cada $n \geq 0$ e $a \in S$. Então $\nu$ é $\phi-\mathrm{DLR}$ se, e somente, se para todo par de cilindros $[\underline{a}]:=\left[a_{0}, a_{1}, \ldots, a_{n-1}\right]$ e $[\underline{b}]:=\left[b_{0}, b_{1}, \ldots, b_{n-1}\right]$ de tamanho $n \in \mathbb{N}$ tais que $a_{n-1}=b_{n-1}$ e $\nu([\underline{a}])>0$, a aplicação $v_{\underline{a}, \underline{b}}:[\underline{a}] \rightarrow[\underline{b}]$ dada por $v_{\underline{a}, \underline{b}}\left(\underline{a} x_{n}^{\infty}\right)=\left(\underline{b} x_{n}^{\infty}\right)$, satisfaz

$$
\frac{\mathrm{d} \nu \circ v_{\underline{a}, \underline{b}}}{\mathrm{~d} \nu}=e^{\phi_{n}\left(\underline{b} \sigma^{n} x\right)-\phi_{n}\left(\underline{a}^{n} x\right)}, \quad \nu \text { - q.t.p. } \operatorname{em} \mathcal{B} \cap[\underline{a}] .
$$

Para a prova da proposição acima precisamos do seguinte lema.

Lema 82. Sejam $(\Omega, \mathcal{F}, \mu)$ um espaço mensurável e $\left\{A_{i}\right\}_{i \geq 1} \subset \mathcal{F}$ uma partição tal que $\mu\left(A_{i}\right)<\infty$ para cada $i \geq 1$. Para cada $f \in L^{1}(\Omega, \mathcal{F}, \mu)$

$$
\mathbb{E}\left[f \mid \sigma\left(A_{1}, A_{2}, \ldots\right)\right](x)=\sum_{\substack{i \geq 1 \\ \mu\left(A_{i}\right)>0}} \mathbb{1}_{A_{i}}(x) \frac{1}{\mu\left(A_{i}\right)} \int_{A_{i}} f(x) \mathrm{d} \mu(x), \quad \mu-\text { q.t.p. }
$$

Demonstração. Definamos

$$
\varphi(x):=\sum_{\substack{i \geq 1 \\ \mu\left(A_{i}\right)>0}} \mathbb{1}_{A_{i}}(x) \frac{1}{\mu\left(A_{i}\right)} \int_{A_{i}} f(x) \mathrm{d} \mu(x),
$$

$\operatorname{logo} \varphi$ é $\sigma\left(A_{1}, A_{2}, \ldots\right)$-mensurável. Para cada $A_{i}$ tal que $\mu\left(A_{i}\right)>0$ temos

$$
\begin{aligned}
\int_{A_{i}} \varphi \mathrm{d} \mu & =\int_{A_{i}}\left(\sum_{\substack{j \geq 1 \\
\mu\left(A_{j}\right)>0}} \mathbb{1}_{A_{j}}(y) \frac{1}{\mu\left(A_{j}\right)} \int_{A_{j}} f(x) \mathrm{d} \mu(x)\right) \mathrm{d} \mu(y) \\
& =\int_{A_{i}}\left(\frac{1}{\mu\left(A_{i}\right)} \int_{A_{i}} f(x) \mathrm{d} \mu(x)\right) \mathrm{d} \mu(y) \\
& =\int_{A_{i}} f(x) \mathrm{d} \mu(x) .
\end{aligned}
$$

Seja $D \in \sigma\left(A_{1}, A_{2}, \ldots\right)$, logo existe $I \subset \mathbb{N}$ tal que $D=\sqcup_{i \in I} A_{i}$. Da Equação $(2.24)$ e da definição da esperança condicional temos

$$
\begin{aligned}
\int_{D} \mathbb{E}\left[f \mid \sigma\left(A_{1}, A_{2}, \ldots\right)\right](x) \mathrm{d} \mu(x) & =\int_{D} f(x) \mathrm{d} \mu(x)=\sum_{i \in I} \int_{A_{i}} f(x) \mathrm{d} \mu(x) \\
& =\sum_{\substack{i \in I \\
\mu\left(A_{i}\right)>0}} \int_{A_{i}} f(x) \mathrm{d} \mu(x) \\
& =\sum_{\substack{i \in I \\
\mu\left(A_{i}\right)>0}} \int_{A_{i}} \varphi(x) \mathrm{d} \mu(x) \\
& =\int_{D} \varphi \mathrm{d} \mu,
\end{aligned}
$$

Desde que $D \in \sigma\left(A_{1}, A_{2}, \ldots\right)$ é arbitrário e $\varphi$ é $\sigma\left(A_{1}, A_{2}, \ldots\right)$-mensurável obtemos a Equação (2.23).

Para cada $n, k \geq 0$ definamos

$$
\sigma_{n}^{n+k} \mathcal{B}:=\sigma\left(\left\{\pi_{n}, \pi_{n+1}, \ldots, \pi_{n+k}\right\}\right),
$$


notemos que $\left\{\sigma^{-n}[\underline{\omega}] \mid \underline{\omega} \in \mathcal{W}_{k+1}\right\}$ é uma partição $\sigma_{n}^{n+k} \mathcal{B}$-mensurável de $\Sigma_{A}$. Logo

$$
\sigma_{n}^{n+k} \mathcal{B}=\sigma\left(\left\{\sigma^{-n}[\underline{\omega}] \mid \underline{\omega} \in \mathcal{W}_{k+1}\right\}\right) .
$$

Consideremos $\nu$ como nas hipóteses da Proposição 81, ou seja, $\nu\left(\pi_{n}^{-1}\{a\}\right)<\infty$ para cada $n \geq 0$ e $a \in S$. Pela Equação (2.23) obtemos

$$
\mathbb{E}\left[f \mid \sigma_{n}^{n+k} \mathcal{B}\right](x)=\frac{1}{\nu\left(\sigma^{-n}\left[x_{n}^{n+k}\right]\right)} \int_{\sigma^{-n}\left[x_{n}^{n+k}\right]} f(y) \mathrm{d} \nu(y), \quad \nu-\text { q.t.p. }
$$

para cada $f \in L^{1}\left(\Sigma_{A}, \mathcal{B}, \nu\right)$ e $n, k \geq 0$ e $x \in \Sigma_{A}$ tal que $\nu\left(\sigma^{-n}\left[x_{n}^{n+k}\right]\right)>0$.

Demonstração. (Da Proposição 81). Seja $\nu$ uma medida $\phi-D L R$ e $\underline{c} \in \mathcal{W}_{m}, m \in \mathbb{N}$, tal que $[\underline{a}, \underline{c}] \neq \emptyset, \log \mathrm{O}$

$$
\begin{aligned}
& \nu \circ v_{a b}([\underline{a}, \underline{c}])=\nu([\underline{b}, \underline{c}])=\int_{[\underline{b}, c]} \mathrm{d} \nu=\int_{\Sigma_{A}} \mathbb{1}_{[\underline{b}, \underline{c}]} \mathrm{d} \nu \\
& =\int_{\Sigma_{A}} \mathbb{1}_{[c]} \circ \sigma^{n}(x) \mathbb{1}_{[b]}(x) \mathrm{d} \nu(x) \\
& =\int_{\Sigma_{A}} \mathbb{1}_{[c]} \circ \sigma^{n}(x) \mathbb{E}\left(\mathbb{1}_{[b]} \mid \sigma^{-n} \mathcal{B}\right)(x) \mathrm{d} \nu(x) \\
& =\int_{\Sigma_{A}} \mathbb{1}_{[\underline{c}]} \circ \sigma^{n}(x) \frac{e^{\phi_{n}\left(\underline{b} \sigma^{n} x\right)}}{\sum_{\left\{\underline{b} \sigma^{n} x \in \Sigma_{A}\right\}}} \mathrm{d} \nu(x) \\
& =\int_{\Sigma_{A}} \mathbb{1}_{[\underline{[}]} \circ \sigma^{n}(x) e^{\phi_{n}\left(\underline{b} \sigma^{n} x\right)-\phi_{n}\left(\underline{a} \sigma^{n} x\right)} \frac{e^{\phi_{n}\left(\underline{a} \sigma^{n} x\right)} \mathbb{1}_{\left\{\underline{a} \sigma^{n} x \in \Sigma_{A}\right\}}}{\sum_{\sigma^{n} y=\sigma^{n} x} e^{\phi_{n}(y)}} \mathrm{d} \nu(x) \\
& =\int_{\Sigma_{A}} \mathbb{1}_{[\underline{c}]} \circ \sigma^{n}(x) e^{\phi_{n}\left(\underline{b} \sigma^{n} x\right)-\phi_{n}\left(\underline{a} \sigma^{n} x\right)} \mathbb{E}\left(\mathbb{1}_{[\underline{a}]} \mid \sigma^{-n} \mathcal{B}\right)(x) \mathrm{d} \nu(x) \\
& =\int_{\Sigma_{A}} \mathbb{1}_{[\underline{c}]} \circ \sigma^{n}(x) e^{\phi_{n}\left(\underline{b} \sigma^{n} x\right)-\phi_{n}\left(\underline{a} \sigma^{n} x\right)} \mathbb{1}_{[a]}(x) \mathrm{d} \nu(x) \\
& =\int_{\Sigma_{A}} \mathbb{1}_{[\underline{a}, \underline{c}]}(x) e^{\phi_{n}\left(\underline{b} \sigma^{n} x\right)-\phi_{n}\left(\underline{a} \sigma^{n} x\right)} \mathrm{d} \nu(x) \\
& =\int_{[\underline{a}, \underline{c}]} e^{\phi_{n}\left(\underline{b} \sigma^{n} x\right)-\phi_{n}\left(\underline{a} \sigma^{n} x\right)} \mathrm{d} \nu(x) .
\end{aligned}
$$

Assim obtemos

$$
\nu \circ v_{\underline{a}, \underline{b}}([\underline{a}, \underline{c}])=\int_{[\underline{a}, \underline{c}]} e^{\phi_{n}\left(\underline{b} \sigma^{n} x\right)-\phi_{n}\left(\underline{a} \sigma^{n} x\right)} \mathrm{d} \nu(x) .
$$

Como $\left\{[\underline{a}, \underline{c}] \mid \underline{c} \in \mathcal{W}_{m}, m \in \mathbb{N}\right\}$ é uma base da topologia induzida em [a] $]$, então pela Equação (2.26)

$$
\nu \circ v_{\underline{a}, \underline{b}}(E)=\int_{E} e^{\phi_{n}\left(\underline{b} \sigma^{n} x\right)-\phi_{n}\left(\underline{a} \sigma^{n} x\right)} \mathrm{d} \nu(x)
$$

para todo $E \in \mathcal{B} \cap[\underline{a}]$. Portanto

$$
\left.\frac{\mathrm{d} \nu \circ v_{\underline{a}, \underline{b}}}{\mathrm{~d} \nu}\right|_{[\underline{a}]}=e^{\phi_{n}\left(\underline{b} \sigma^{n} x\right)-\phi_{n}\left(\underline{a} \sigma^{n} x\right)}, \quad \nu-\text { q.t.p. em }[\underline{a}] .
$$

Sejam $[\underline{a}]:=\left[a_{0}, a_{1}, \ldots, a_{n-1}\right],[\underline{b}]:=\left[b_{0}, b_{1}, \ldots, b_{n-1}\right]$ duas palavras de tamanho $n$ e $c \in S$ tais que $A\left(a_{n-1}, c\right)=A\left(b_{n-1}, c\right)=1$ e $\nu([\underline{a}, c])>0$. Para cada $x \in \sigma^{-n}[c]$ tal que $\nu\left(\left[\underline{a}, x_{n}^{n+k}\right]\right)>0$, $k \geq 0$, vamos calcular o seguinte limite $\frac{\nu\left[\underline{b}, x_{n}^{n+k}\right]}{\nu\left[\underline{a}, x_{n}^{n+k}\right]}$ quando $k \rightarrow \infty$ de duas maneiras diferentes: 
a.) Notemos que $\mathbb{1}_{[\underline{a}]}, \mathbb{1}_{[\underline{b}]} \in L^{1}\left(\Sigma_{A}, \mathcal{B}, \nu\right)$ e $\sigma^{-n} \mathcal{B}=\sigma\left(\cup_{k \geq 0} \sigma_{n}^{n+k} \mathcal{B}\right)$. Usando a Equação (2.25) e o Teorema 76 temos

$$
\begin{aligned}
\lim _{k \rightarrow \infty} \frac{\nu\left[\underline{b}, x_{n}^{n+k}\right]}{\nu\left[\underline{a}, x_{n}^{n+k}\right]} & =\lim _{k \rightarrow \infty} \frac{\frac{\nu\left[\underline{b}, x_{n}^{n+k}\right]}{\nu\left(\sigma^{-n}\left[x_{n}^{n+k}\right]\right)}}{\frac{\nu\left[a, x_{n}^{n+k}\right]}{\nu\left(\sigma^{-n}\left[x_{n}^{n+k}\right]\right)}} \\
& =\lim _{k \rightarrow \infty} \frac{\mathbb{E}\left[\mathbb{1}_{[\underline{b}]} \mid \sigma_{n}^{n+k} \mathcal{B}\right](x)}{\mathbb{E}\left[\mathbb{1}_{[\underline{a}]} \mid \sigma_{n}^{n+k} \mathcal{B}\right](x)} \\
& =\frac{\mathbb{E}\left[\mathbb{1}_{[\underline{b}]} \mid \sigma^{-n} \mathcal{B}\right](x)}{\mathbb{E}\left[\mathbb{1}_{[\underline{a}]} \mid \sigma^{-n} \mathcal{B}\right](x)}, \quad \nu \text { - q.t.p.. }
\end{aligned}
$$

b.) Pela Equação (2.22) temos

$$
\begin{aligned}
& \int_{\Sigma_{A}} e^{\phi_{n+1}\left(\underline{b} c \sigma^{n+1} x\right)-\phi_{n+1}\left(\underline{a} c \sigma^{n+1} x\right)} \mathbb{1}_{[\underline{a} c]}(x) \mathrm{d} \nu(x)=\int_{[\underline{a} c]} e^{\phi_{n+1}\left(\underline{b} c \sigma^{n+1} x\right)-\phi_{n+1}\left(\underline{a} c \sigma^{n+1} x\right)} \mathrm{d} \nu(x) \\
&=\nu([\underline{b} c])<\infty, \\
& \log \mathrm{O}, e^{\phi_{n+1}\left(\underline{b} c \sigma^{n+1}(\cdot)\right)-\phi_{n+1}\left(\underline{a} c \sigma^{n+1}(\cdot)\right)} \mathbb{1}_{[\underline{a} c]}(\cdot) \in L^{1}\left(\Sigma_{A}, \mathcal{B}, \nu\right) .
\end{aligned}
$$

Notemos que $\mathcal{B}=\sigma\left(\cup_{k \geq 0} \sigma_{0}^{n+k} \mathcal{B}\right)$. Usando as Equações (2.22), (2.25) e o Teorema 76 temos

$$
\begin{aligned}
\lim _{k \rightarrow \infty} \frac{\nu\left[\underline{b}, x_{n}^{n+k}\right]}{\nu\left[\underline{a}, x_{n}^{n+k}\right]} & =\lim _{k \rightarrow \infty} \frac{\left(\nu \circ v_{\underline{a}, \underline{b} c}\right)\left[\underline{a}, x_{n}^{n+k}\right]}{\nu\left[\underline{a}, x_{n}^{n+k}\right]} \\
& =\lim _{k \rightarrow \infty} \frac{1}{\nu\left[\underline{a}, x_{n}^{n+k}\right]} \int_{\left[\underline{a}, x_{n}^{n+k}\right]} e^{\phi_{n+1}\left(\underline{b} c \sigma^{n+1} y\right)-\phi_{n+1}\left(\underline{a} c \sigma^{n+1} y\right)} \mathrm{d} \nu(y) \\
& =\lim _{k \rightarrow \infty} \frac{1}{\nu\left[\underline{a}, x_{n}^{n+k}\right]} \int_{\left[\underline{a}, x_{n}^{n+k}\right]} e^{\phi_{n+1}\left(\underline{b} c \sigma^{n+1} y\right)-\phi_{n+1}\left(\underline{a} c \sigma^{n+1} y\right)} \mathbb{1}_{[\underline{a c}]}(y) \mathrm{d} \nu(y) \\
& =\lim _{k \rightarrow \infty} \mathbb{E}\left[e^{\phi_{n+1}\left(\underline{b} c \sigma^{n+1} y\right)-\phi_{n+1}\left(\underline{a} c \sigma^{n+1} y\right)} \mathbb{1}_{[\underline{a} c]}(y) \mid \sigma_{0}^{n+k} \mathcal{B}\right]\left(\underline{a} x_{n}^{\infty}\right) \\
& =\mathbb{E}\left[e^{\phi_{n+1}\left(\underline{b} c \sigma^{n+1} y\right)-\phi_{n+1}\left(\underline{a} c \sigma^{n+1} y\right)} \mathbb{1}_{[\underline{a} c]}(y) \mid \mathcal{B}\right]\left(\underline{a} x_{n}^{\infty}\right) \\
& =e^{\phi_{n+1}\left(\underline{b} c x_{n+1}^{\infty}\right)-\phi_{n+1}\left(\underline{a} c x_{n+1}^{\infty}\right)} \\
& =e^{\phi_{n}\left(\underline{b} \sigma^{n} x\right)-\phi_{n}\left(\underline{a} \sigma^{n} x\right)}, \quad \nu-\text { q.t.p... }
\end{aligned}
$$

De $(2.27)$ e (2.28) obtemos

$$
\mathbb{E}\left[\mathbb{1}_{[\underline{b}]} \mid \sigma^{-n} \mathcal{B}\right](x)=e^{\phi_{n}\left(\underline{b} \sigma^{n} x\right)-\phi_{n}\left(\underline{a} \sigma^{n} x\right)} \mathbb{E}\left[\mathbb{1}_{[\underline{a}]} \mid \sigma^{-n} \mathcal{B}\right](x), \quad \nu-\text { q.t.p. }
$$

onde $x_{n}=c$ e $A\left(a_{n-1}, c\right)=A\left(b_{n-1}, c\right)=1$. Fixando a palavra $\underline{a}$ e deixando livre $\underline{b}$, somamos na Equação (2.29) sobre todas as palavras de tamanho $n$ que conectam com $x_{n}$, logo obtemos

$$
\begin{aligned}
\mathbb{E}\left[\mathbb{1}_{[\underline{a}]} \mid \sigma^{-n} \mathcal{B}\right](x) & =\frac{e^{\phi_{n}\left(\underline{a} \sigma^{n} x\right)} \mathbb{E}\left[\mathbb{1}_{\pi_{n}^{-1}\{c\}} \mid \sigma^{-n} \mathcal{B}\right](x)}{\sum_{\sigma^{n} y=\sigma^{n} x} e^{\phi_{n}(y)}} \\
& =\frac{e^{\phi_{n}\left(\underline{a} \sigma^{n} x\right)} \mathbb{1}_{\pi_{n}^{-1}\{c\}}(x)}{\sum_{\sigma^{n} y=\sigma^{n} x} e^{\phi_{n}(y)}} \\
& =\frac{e^{\phi_{n}\left(\underline{a} \sigma^{n} x\right)} \mathbb{1}_{\left\{\underline{a} \sigma^{n} x \in \Sigma_{A}, x_{n}=c\right\}}}{\sum_{\sigma^{n} y=\sigma^{n} x} e^{\phi_{n}(y)}}, \quad \nu-\text { q.t.p.. }
\end{aligned}
$$


Para todo $x \in \Sigma_{A}$ temos

$$
\begin{aligned}
\mathbb{E}\left[\mathbb{1}_{[\underline{a}]} \mid \sigma^{-n} \mathcal{B}\right](x) & =\sum_{c \in S} \mathbb{1}_{\left\{x_{n}=c\right\}} \mathbb{E}\left[\mathbb{1}_{[a]} \mid \sigma^{-n} \mathcal{B}\right](x) \\
& =\sum_{c \in S} \frac{e^{\phi_{n}\left(\underline{a} \sigma^{n} x\right)} \mathbb{1}_{\left\{\underline{a} \sigma^{n} x \in \Sigma_{A}, x_{n}=c\right\}}}{\sum_{\sigma^{n} y=\sigma^{n} x} e^{\phi_{n}(y)}} \\
& =\frac{e^{\phi_{n}\left(\underline{a} \sigma^{n} x\right)} \mathbb{1}_{\left\{\underline{a} \sigma^{n} x \in \Sigma_{A},\right\}}}{\sum_{\sigma^{n} y=\sigma^{n} x} e^{\phi_{n}(y)}}, \quad \nu-\text { q.t.p. },
\end{aligned}
$$

portanto $\nu$ é uma medida $\phi-$ DLR.

Note que na prova da proposição acima o fato de $\nu$ ser uma medida tais que $\nu\left(\pi_{n}^{-1}\{a\}\right)<\infty$ para cada $n \geq 0$ e $a \in S$ é usado somente para demonstrar a recíproca. A prova da implicação é sempre verdadeira para qualquer medida DLR. A seguir temos algumas propriedades da derivada de Radon-Nikodým que serão usadas na prova do Teorema 84 e a Proposição 88.

Lema 83. Sejam $(\Omega, \mathcal{F})$ um espaço mensurável e $\mu_{1}, \mu_{2}, \mu_{3}$ medidas $\sigma$-finitas em $\mathcal{F}$. Logo:

i.) Se $\mu_{1} \sim \mu_{2}$, então

$$
\frac{\mathrm{d} \mu_{2}}{\mathrm{~d} \mu_{1}} \neq 0, \mu_{1}-\text { q.t.p. } \quad e \quad \frac{\mathrm{d} \mu_{1}}{\mathrm{~d} \mu_{2}}=\left(\frac{\mathrm{d} \mu_{2}}{\mathrm{~d} \mu_{1}}\right)^{-1}, \quad \mu_{1} \text { ou } \mu_{2}-\text { q.t.p. }
$$

ii.) Se $\mu_{1} \ll \mu_{2}$ e $T$ é uma aplicação invertivel tal que $T$ e $T^{-1}$ são mensuráveis, então

$$
\frac{\mathrm{d} \mu_{1} \circ T}{\mathrm{~d} \mu_{2} \circ T}=\frac{\mathrm{d} \mu_{1}}{\mathrm{~d} \mu_{2}} \circ T, \quad \mu_{2} \circ T-\text { q.t.p. }
$$

iii.) Se $\mu_{1} \ll \mu_{2} \ll \mu_{3}$, então

$$
\frac{\mathrm{d} \mu_{1}}{\mathrm{~d} \mu_{2}} \cdot \frac{\mathrm{d} \mu_{2}}{\mathrm{~d} \mu_{3}}=\frac{\mathrm{d} \mu_{1}}{\mathrm{~d} \mu_{3}}, \quad \mu_{3}-\text { q.t.p.. }
$$

Demonstração. Só vamos mostrar o item ii.). Pela derivada de Radon-Nikodým, existe uma função mensurável $h=\frac{\mathrm{d} \mu_{1} \circ T}{\mathrm{~d} \mu_{2} \circ T}, \mu_{2} \circ T-$ q.t.p.. Assim,

$$
\mu_{1} \circ T(E)=\int_{E} h \mathrm{~d} \mu_{2} \circ T, \quad \forall E \in \mathcal{F},
$$

escolhendo $E=T^{-1}(F)$, onde $F \in \mathcal{F}$, obtemos

$$
\begin{aligned}
\mu_{1}(F) & =\mu_{1} \circ T\left(T^{-1} F\right)=\int_{T^{-1}(F)} h \mathrm{~d} \mu_{2} \circ T \\
& =\int_{F} h \circ T^{-1} \mathrm{~d} \mu_{2}, \quad \forall F \in \mathcal{F} .
\end{aligned}
$$

Portanto $h \circ T^{-1}=\frac{\mathrm{d} \mu_{1}}{\mathrm{~d} \mu_{2}}$, e como $T$ é invertível obtemos o a Equação (2.31).

Teorema 84. Sejam $\Sigma_{A}$ um shift de Markov e $\phi: \Sigma_{A} \rightarrow \mathbb{R}$ um potencial mensurável. Se $\nu$ é uma medida $\sigma$-finita, não-singular e $(\phi, \lambda)$-conforme para algum $\lambda>0$, então para qualquer par de cilindros $[\underline{a}]:=\left[a_{0}, a_{1}, \ldots, a_{n-1}\right]$ e $[\underline{b}]:=\left[b_{0}, b_{1}, \ldots, b_{n-1}\right]$ de tamanho $n \in \mathbb{N}$ tais que $a_{n-1}=b_{n-1}$ e $\nu([\underline{a}])>0$ temos que a aplicação $v_{\underline{a}, \underline{b}}:[\underline{a}] \rightarrow[\underline{b}]$ satisfaz

$$
\frac{\mathrm{d} \nu \circ v_{\underline{a}, \underline{b}}}{\mathrm{~d} \nu}=e^{\phi_{n}\left(\underline{b} \sigma^{n} x\right)-\phi_{n}\left(\underline{a} \sigma^{n} x\right)}, \quad \nu \text { - q.t.p. em } \mathcal{B} \cap[\underline{a}] .
$$


Demonstração. Sejam $[\underline{a}]$ e $[\underline{b}]$ dois cilindros de tamanho $n$ satisfazendo as hipóteses. Notemos que a aplicação $v_{\underline{a}, \underline{b}}:[\underline{a}] \rightarrow[\underline{b}]$ satisfaz a seguinte relação

$$
v_{\underline{a}, \underline{b}}=\left.I_{\underline{b}} \circ \sigma^{n}\right|_{[\underline{a}]}
$$

onde $I_{\underline{b}}: \sigma^{n}[\underline{b}] \rightarrow[\underline{b}], x \mapsto \underline{b} x$. Notemos que

i.) $\left.\nu \sim \nu \circ \sigma^{n}\right|_{[\underline{a}]} \operatorname{em~} \mathcal{B} \cap[\underline{a}]$,

ii.) $\nu \circ v_{\underline{a}, \underline{b}} \ll \nu \operatorname{em} \mathcal{B} \cap[\underline{a}]$.

Como $\nu$ é conforme o item i.) segue. O item ii.) é consequência do item $i$.) e do fato que $\nu$ é não-singular. Daqui em diante denotaremos $\sigma^{n}:=\left.\sigma\right|_{[\underline{a}]} ^{n}$. Pelo item $i$.) e ii.) temos

$$
\left.\frac{\mathrm{d} \nu \circ v_{\underline{a}, \underline{b}}}{\mathrm{~d} \nu}\right|_{[\underline{a}]}=\left.\left.\frac{\mathrm{d} \nu \circ v_{\underline{a}, \underline{b}}}{\mathrm{~d} \nu \circ \sigma^{n}}\right|_{[\underline{a}]} \cdot \frac{\mathrm{d} \nu \circ \sigma^{n}}{\mathrm{~d} \nu}\right|_{[\underline{a}]}, \quad \nu-\text { q.t.p. em }[\underline{a}] .
$$

Usando a Equação (2.32)

$$
\begin{aligned}
\frac{\mathrm{d} \nu \circ v_{\underline{a}, \underline{b}}}{\left.\mathrm{~d} \nu \circ \sigma^{n}\right|_{[\underline{a}]}} & =\left.\frac{\mathrm{d} \nu \circ I_{\underline{b}} \circ \sigma^{n}}{\mathrm{~d} \nu \circ \sigma^{n}}\right|_{[\underline{a}]} \\
& =\left.\frac{\mathrm{d} \nu \circ I_{\underline{b}}}{\mathrm{~d} \nu} \circ \sigma^{n}\right|_{[\underline{a}]}, \quad \nu \circ \sigma^{n}-\text { q.t.p. em }[\underline{a}] \\
& =\left.\frac{\mathrm{d} \nu \circ I_{\underline{b}}}{\mathrm{~d} \nu} \circ \sigma^{n}\right|_{[\underline{a}]}, \quad \nu-\text { q.t.p. em }[\underline{a}] .
\end{aligned}
$$

De $(2.33)$ e $(2.34)$ temos

$$
\left.\frac{\mathrm{d} \nu \circ v_{\underline{a}, \underline{b}}}{\mathrm{~d} \nu}\right|_{[\underline{a}]}=\left.\left.\frac{\mathrm{d} \nu \circ I_{\underline{b}}}{\mathrm{~d} \nu} \circ \sigma^{n}\right|_{[\underline{a}]} \cdot \frac{\mathrm{d} \nu \circ \sigma^{n}}{\mathrm{~d} \nu}\right|_{[\underline{a}]}, \quad \nu-\text { q.t.p. em }[\underline{a}] .
$$

A seguir vamos calcular cada derivada de Radon-Nikodým da parte direita da Equação (2.35).

a.) Calculando $\left.\frac{\mathrm{d} \nu \circ I_{\underline{b}}}{\mathrm{~d} \nu} \circ \sigma^{n}\right|_{[\underline{a}]}$ por indução em $n$

- Sejam $a, b \in S$ tal que $a=b$, e $x \in[a]$, logo

$$
\begin{aligned}
\frac{\mathrm{d} \nu \circ I_{b}}{\mathrm{~d} \nu} \circ \sigma(x) & =\frac{\mathrm{d} \nu \circ I_{b}}{\mathrm{~d} \nu}(\sigma(x))=\frac{\mathrm{d} \nu \circ I_{b}}{\mathrm{~d} \nu \circ \sigma \circ I_{b}}(\sigma(x)) \\
& =\frac{\mathrm{d} \nu}{\mathrm{d} \nu \circ \sigma} \circ I_{b}(\sigma(x))=\frac{\mathrm{d} \nu}{\mathrm{d} \nu \circ \sigma}\left(b x_{1}^{\infty}\right), \quad \nu \circ \sigma-\text { q.t.p. em }[a] \\
& =\lambda^{-1} e^{\phi\left(b x_{1}^{\infty}\right)}, \quad \nu-\text { q.t.p. em }[a] .
\end{aligned}
$$

- Sejam $[\underline{a}]=\left[a_{0}, a_{1}\right]$ e $[\underline{b}]=\left[b_{0}, b_{1}\right]$ tal que $a_{1}=b_{1}$, logo

$$
\begin{aligned}
\left.\frac{\mathrm{d} \nu \circ I_{b_{0} b_{1}}}{\mathrm{~d} \nu} \circ \sigma^{2}\right|_{[a]} & =\left.\frac{\mathrm{d} \nu \circ I_{b_{0}} \circ I_{b_{1}}}{\mathrm{~d} \nu} \circ \sigma^{2}\right|_{[\underline{a}]} \\
& =\left.\left.\frac{\mathrm{d} \nu \circ I_{b_{0}} \circ I_{b_{1}}}{\mathrm{~d} \nu \circ I_{b_{1}}} \circ \sigma^{2}\right|_{[\underline{a}]} \cdot \frac{\mathrm{d} \nu \circ I_{b_{1}}}{\mathrm{~d} \nu} \circ \sigma^{2}\right|_{[\underline{a}]}, \quad \nu-\text { q.t.p. em }[\underline{a}] .
\end{aligned}
$$

Assim para cada $x \in[\underline{a}]$ temos

$$
\frac{\mathrm{d} \nu \circ I_{b_{0} b_{1}}}{\mathrm{~d} \nu}\left(\sigma^{2}(x)\right)=\frac{\mathrm{d} \nu \circ I_{b_{0}} \circ I_{b_{1}}}{\mathrm{~d} \nu \circ I_{b_{1}}}\left(\sigma^{2}(x)\right) \frac{\mathrm{d} \nu \circ I_{b_{1}}}{\mathrm{~d} \nu}\left(\sigma^{2}(x)\right), \quad \nu-\text { q.t.p. em }[\underline{a}] .(2.37)
$$


Pelo item ii.) do Lema 83 temos $\frac{\mathrm{d} \nu \circ I_{b_{0}} \circ I_{b_{1}}}{\mathrm{~d} \nu \circ I_{b_{1}}}(y)=\frac{\mathrm{d} \nu \circ I_{b_{0}}}{\mathrm{~d} \nu}\left(I_{b_{1}}(y)\right), \nu \circ I_{b_{1}}-$ q.t.p. em $\sigma^{2}[\underline{a}]$, isto é, se

$$
E=\left\{y \in \sigma^{2}[\underline{a}] \mid \frac{\mathrm{d} \nu \circ I_{b_{0}} \circ I_{b_{1}}}{\mathrm{~d} \nu \circ I_{b_{1}}}(y) \neq \frac{\mathrm{d} \nu \circ I_{b_{0}}}{\mathrm{~d} \nu}\left(I_{b_{1}}(y)\right)\right\},
$$

então $\nu \circ I_{b_{1}}(E)=0, \operatorname{logo} \nu(E)=0$. Do fato de $\nu$ ser não-singular temos $\nu\left(\sigma^{-2} E\right)=0$, assim

$$
\begin{aligned}
\left.\sigma\right|_{[\underline{a}]} ^{-2} E & =\left\{x \in[\underline{a}] \mid \frac{\mathrm{d} \nu \circ I_{b_{0}} \circ I_{b_{1}}}{\mathrm{~d} \nu \circ I_{b_{1}}}\left(\left.\sigma\right|_{[\underline{a}]} ^{2} x\right) \neq \frac{\mathrm{d} \nu \circ I_{b_{0}}}{\mathrm{~d} \nu}\left(I_{b_{1}}\left(\left.\sigma\right|_{[a]} ^{2} x\right)\right)\right\} \\
& =\left\{x \in[\underline{a}] \mid \frac{\mathrm{d} \nu \circ I_{b_{0}} \circ I_{b_{1}}}{\mathrm{~d} \nu \circ I_{b_{1}}}\left(\sigma^{2} x\right) \neq \frac{\mathrm{d} \nu \circ I_{b_{0}}}{\mathrm{~d} \nu}\left(I_{b_{1}}\left(\sigma^{2} x\right)\right)\right\}
\end{aligned}
$$

tem medida nula, isto é,

$$
\frac{\mathrm{d} \nu \circ I_{b_{0}} \circ I_{b_{1}}}{\mathrm{~d} \nu \circ I_{b_{1}}}\left(\sigma^{2}(x)\right)=\frac{\mathrm{d} \nu \circ I_{b_{0}}}{\mathrm{~d} \nu}\left(I_{b_{1}}\left(\sigma^{2}(x)\right)\right) \quad \nu-\text { q.t.p. em }[\underline{a}] .
$$

Substituindo na Equação (2.37) obtemos

$$
\begin{aligned}
\frac{\mathrm{d} \nu \circ I_{b_{0} b_{1}}}{\mathrm{~d} \nu}\left(\sigma^{2}(x)\right) & =\frac{\mathrm{d} \nu \circ I_{b_{0}}}{\mathrm{~d} \nu}\left(I_{b_{1}}\left(\sigma^{2}(x)\right)\right) \cdot \frac{\mathrm{d} \nu \circ I_{b_{1}}}{\mathrm{~d} \nu}\left(\sigma^{2}(x)\right) \\
& =\frac{\mathrm{d} \nu \circ I_{b_{0}}}{\mathrm{~d} \nu}\left(b_{1} x_{2}^{\infty}\right) \cdot \frac{\mathrm{d} \nu \circ I_{b_{1}}}{\mathrm{~d} \nu}\left(x_{2}^{\infty}\right), \quad \nu-\text { q.t.p. em }[\underline{a}] .
\end{aligned}
$$

Por (2.36) temos

$$
\begin{aligned}
\frac{\mathrm{d} \nu \circ I_{b_{0}}}{\mathrm{~d} \nu}\left(b_{1} x_{2}^{\infty}\right) & =\frac{\mathrm{d} \nu \circ I_{b_{0}}}{\mathrm{~d} \nu}\left(\sigma\left(b_{0} b_{1} x_{2}^{\infty}\right)\right) \\
& =\lambda^{-1} e^{\phi\left(b_{0} b_{1} x_{2}^{\infty}\right)}, \quad \nu-\text { q.t.p. em }[\underline{b}],
\end{aligned}
$$

$\log 0$

$$
F=\left\{x \in[\underline{b}] \mid \frac{\mathrm{d} \nu \circ I_{b_{0}}}{\mathrm{~d} \nu}\left(b_{1} x_{2}^{\infty}\right) \neq \lambda^{-1} e^{\phi\left(b_{0} b_{1} x_{2}^{\infty}\right)}\right\}
$$

tem medida nula. Como $\nu(F)=0$, então $\nu\left(\left.\sigma\right|_{[b]} ^{2} F\right)=0$. Pelo fato de $\nu$ ser não-singular temos $\nu\left(\left.\left.\sigma\right|_{[\underline{a}]} ^{-2} \sigma\right|_{[\underline{b}]} ^{2} F\right)=0$, é dizer,

$$
\left.\left.\sigma\right|_{[\underline{a}]} ^{-2} \sigma\right|_{[\underline{b}]} ^{2} F=\left\{x \in[\underline{a}] \mid \frac{\mathrm{d} \nu \circ I_{b_{0}}}{\mathrm{~d} \nu}\left(b_{1} x_{2}^{\infty}\right) \neq \lambda^{-1} e^{\phi\left(b_{0} b_{1} x_{2}^{\infty}\right)}\right\}
$$

tem medida nula. Logo

$$
\frac{\mathrm{d} \nu \circ I_{b_{0}}}{\mathrm{~d} \nu}\left(b_{1} x_{2}^{\infty}\right)=\lambda^{-1} e^{\phi\left(b_{0} b_{1} x_{2}^{\infty}\right)}, \quad \nu-\text { q.t.p. em }[\underline{a}] .
$$

De maneira análoga

$$
\frac{\mathrm{d} \nu \circ I_{b_{1}}}{\mathrm{~d} \nu}\left(x_{2}^{\infty}\right)=\lambda^{-1} e^{\phi\left(b_{1} x_{2}^{\infty}\right)}, \quad \nu-\text { q.t.p. em }[\underline{a}] .
$$

Substituindo as Equações (2.39) e (2.40) em (2.38) temos

$$
\begin{aligned}
\frac{\mathrm{d} \nu \circ I_{b_{0} b_{1}}}{\mathrm{~d} \nu}\left(\sigma^{2}(x)\right) & =\lambda^{-1} e^{\phi\left(b_{0} b_{1} x_{2}^{\infty}\right)} \lambda^{-1} e^{\phi\left(b_{1} x_{2}^{\infty}\right)} \\
& =\lambda^{-2} e^{\phi_{2}\left(b_{0} b_{1} x_{2}^{\infty}\right)}, \quad \nu \text { - q.t.p. em }[\underline{a}]
\end{aligned}
$$


Assim por indução em $n$ obtemos

$$
\frac{\mathrm{d} \nu \circ I_{\underline{b}}}{\mathrm{~d} \nu}\left(\sigma^{n}(x)\right)=\lambda^{-n} e^{\phi_{n}\left(\underline{b} x_{n}^{\infty}\right)}, \quad \nu-\text { q.t.p. em }[\underline{a}] .
$$

b.) Calculando $\left.\frac{\mathrm{d} \nu \circ \sigma^{n}}{\mathrm{~d} \nu}\right|_{[a]}$

$$
\begin{aligned}
\left.\frac{\mathrm{d} \nu \circ \sigma^{n}}{\mathrm{~d} \nu}\right|_{[\underline{a}]} & =\left.\frac{\mathrm{d} \nu \circ \sigma^{n}}{\mathrm{~d} \nu \circ I_{\underline{a}} \circ \sigma^{n}}\right|_{[\underline{a}]} \\
& =\left.\frac{\mathrm{d} \nu}{\mathrm{d} \nu \circ I_{\underline{a}}} \circ \sigma^{n}\right|_{[a]}, \quad \nu \circ I_{\underline{a}} \circ \sigma^{n}-\text { q.t.p. em }[\underline{a}] \\
& =\left.\frac{d \nu}{d \nu \circ I_{\underline{a}}} \circ \sigma^{n}\right|_{[\underline{a}]}, \quad \nu-\text { q.t.p. em }[\underline{a}] .
\end{aligned}
$$

Desde que $\nu \sim \nu \circ I_{\underline{a}}$ em $\mathcal{B} \cap \sigma^{n}[\underline{a}]$, então para $x \in[\underline{a}]$ temos

$$
\begin{aligned}
\frac{\mathrm{d} \nu \circ \sigma^{n}}{\mathrm{~d} \nu}(x) & =\frac{\mathrm{d} \nu}{\mathrm{d} \nu \circ I_{\underline{a}}}\left(\sigma^{n}(x)\right) \\
& =\left(\frac{\mathrm{d} \nu \circ I_{\underline{a}}}{\mathrm{~d} \nu}\right)^{-1}\left(\sigma^{n}(x)\right), \quad \nu-\text { q.t.p. em }[\underline{a}] \\
& =\lambda^{n} e^{-\phi_{n}\left(\underline{a} x_{n}^{\infty}\right)}, \quad \nu-\text { q.t.p. em }[\underline{a}]
\end{aligned}
$$

Substituindo as Equações (2.41) e (2.42) na Equação (2.35) obtemos

$$
\frac{\mathrm{d} \nu \circ v_{\underline{a}, \underline{b}}}{\mathrm{~d} \nu}(x)=e^{\phi_{n}\left(\underline{b} \sigma^{n} x\right)-\phi_{n}\left(\underline{a} \sigma^{n} x\right)}, \quad \nu \text { - q.t.p. em }[\underline{a}] .
$$

O seguinte corolário diz que toda medida conforme é DLR, este é uma generalização para o caso $\sigma$-finito do Teorema 68. A prova é consequência da Proposição 81 e do Teorema 84.

Corolário 85. Sejam $\Sigma_{A}$ um shift de Markov, $\phi: \Sigma_{A} \rightarrow \mathbb{R}$ um potencial mensurável satisfazendo a condição $\left\|L_{\phi} \mathbb{1}\right\|_{\infty}<\infty$. Se $\nu$ é uma medida não-singular $e(\phi, \lambda)$-conforme, para algum $\lambda>0$, tais que $\nu([a])<\infty$ para cada $a \in S$ então $\nu$ é $\phi-\mathrm{DLR}$.

O. Sarig estuda a existência de medidas conformes pelos modos de recorrência do potencial, veja [Sa5]. Consideremos $\Sigma_{A}$ um shift topologicamente mixing e $\phi: \Sigma_{A} \rightarrow \mathbb{R}$ um potencial que satisfaz a condição de Walters tal que $P_{G}(\phi)<\infty$. Quando $\phi$ é recorrente, pelo Teorema Generalizado de Ruelle-Perron-Frobenius existe uma medida $\nu$ que é $\left(\phi, e^{P_{G}(\phi)}\right)$-conforme e dá massa positiva aos cilindros. Se impomos a condição $\left\|L_{\phi} \mathbb{1}\right\|_{\infty}<\infty$ então pela Proposição 78 e Corolário 85 temos que $\nu$ é $\phi$-DLR. Por outro lado, se o potencial é $\lambda$-transiente, para algum $\lambda>0$, com variação somável e o shift de Markov é transitivo e localmente compacto, temos que existe uma medida $(\phi, \lambda)$-conforme, veja Teorema 46. De maneira análoga impondo a condição $\left\|L_{\phi} \mathbb{1}\right\|_{\infty}<\infty$ temos que esta medida é $\phi-D L R$. Notemos que a medida conforme do Exemplo 37 é uma medida DLR infinita.

A seguir temos uma consequência do Teorema de Ruelle-Perron-Frobenius Generalizado.

Proposição 86. Sejam $\Sigma_{A}$ shift topologicamente mixing e $\phi: \Sigma_{A} \rightarrow \mathbb{R}$ um potencial que satisfaz a condição de Walters com $P_{G}(\phi)<\infty$. Se $\phi$ é um potencial recorrente então $h \mathrm{~d} \nu$ é $(\phi+\log h-\log h \circ \sigma)-\mathrm{DLR}$, onde $h$ é uma função positiva contínua tal que $L_{\phi} h=e^{P_{G}(\phi)} h e$ $\nu$ é uma medida tal que $L_{\phi}^{*} \nu=e^{P_{G}(\phi)} \nu$. 
Demonstração. Consideremos $m=h \mathrm{~d} \nu$. Pelo Teorema de Ruelle-Perron-Frobenius Generalizado temos que $h$ é uma função continua afastada do zero e do infinito em cilindros e $m$ é uma medida $\sigma$-invariante tal que $m\left(\Sigma_{A}\right)<\infty$ quando $\phi$ é positivamente recorrente e $m\left(\Sigma_{A}\right)=\infty$ caso contrário.

Seja $f \in L^{1}(m), \operatorname{logo}$

$$
\begin{aligned}
e^{P_{G}(\phi)} \int f \mathrm{~d} m & =e^{P_{G}(\phi)} \int f h \mathrm{~d} \nu \\
& =\int L_{\phi}(f h) \mathrm{d} \nu \\
& =\int \sum_{\sigma y=x} e^{\phi(y)} f(y) h(y) \mathrm{d} \nu(x) \\
& =\int \sum_{\sigma y=x} e^{\phi(y)+\log h(y)-\log h(\sigma y)} f(y) h(x) \mathrm{d} \nu(x) \\
& =\int h(x) \sum_{\sigma y=x} e^{(\phi+\log h-\log h \circ \sigma)(y)} f(y) \mathrm{d} \nu(x) \\
& =\int\left(L_{(\phi+\log h-\log h \circ \sigma)} f\right)(x) \mathrm{d} m(x) .
\end{aligned}
$$

portanto $m$ é $\left((\phi+\log h-\log h \circ \sigma), e^{P_{G}(\phi)}\right)$-conforme. Desde que $m$ é invariante e $m([a])<\infty$ temos que $m\left(\pi_{n}^{-1}\{a\}\right)<\infty$ para todo $n \geq 0$ e $a \in S$ logo pelo Corolário 85 temos que $h \mathrm{~d} \nu$ é $(\phi+\log h-\log h \circ \sigma)-$ DLR.

Daqui em diante estamos interessados em provar a recíproca do Corolário 85, ou seja, quando uma medida DLR é conforme. Isso será mostrado no Teorema 89, antes mostraremos dois resultados que serão necessários.

Lema 87. Sejam $\Sigma_{A}$ um shift de Markov, $\phi: \Sigma_{A} \rightarrow \mathbb{R}$ um potencial mensurável e $\mu$ uma medida $\phi-$ DLR não-singular. Sejam $a, c \in S$ tais que $A(a, c)=1$. Se $\mu([a c])>0$, então

i.) Para todo $b \in S$ tal que $A(b, c)=1$ temos $\mu \circ v_{a c, b c} \sim \mu$ em $\mathcal{B} \cap[a c]$.

ii.) $\left.\mu \circ \sigma\right|_{[a c]} \sim \mu e m \mathcal{B} \cap[a c]$.

iii.) Para todo $b \in S$ tal que $A(b, c)=1$ temos $\mu \circ I_{b} \sim \mu$ em $\mathcal{B} \cap[c]$.

Por outro lado se $\mu([a c])=0$, então $\mu([c])=0$.

Demonstração. O item $i$.) é consequência da Proposição 81. A seguir mostraremos o item ii.). Seja $E \in \mathcal{B} \cap[a c]$ tal que $\mu\left(\left.\sigma\right|_{[a c]} E\right)=0$, logo pela não-singularidade de $\mu$ temos $\mu\left(\left.\sigma^{-1} \sigma\right|_{[a c]} E\right)=0$. Assim, $\mu(E)=0$ pois $\mu(E) \leq \mu\left(\left.\sigma^{-1} \sigma\right|_{[a c]} E\right)$. Portanto $\left.\mu \ll \mu \circ \sigma\right|_{[a c]}$ em $\mathcal{B} \cap[a c]$.

Seja $E_{a} \in \mathcal{B} \cap[a c]$ tal que $\mu\left(E_{a}\right)=0$, para $b \in S$ tal que $A(b, c)=1$ definamos $E_{b}:=v_{a c, b c}\left(E_{a}\right)$. Notemos que $E_{b} \in \mathcal{B} \cap[b c]$, logo pela Proposição 81 temos $\mu\left(E_{b}\right)=0$. Como

$$
\mu\left(\left.\sigma^{-1} \sigma\right|_{[a c]} E_{a}\right)=\mu\left(\bigcup_{A(b, c)=1} v_{a c, b c}\left(E_{a}\right)\right)=\mu\left(\bigcup_{A(b, c)=1} E_{b}\right)=\sum_{A(b, c)=1} \mu\left(E_{b}\right)=0,
$$

temos $\mu\left(\left.\sigma\right|_{[a c]} E_{a}\right)=0$, assim obtemos que $\left.\mu \circ \sigma\right|_{[a c]} \ll \mu$ em $\mathcal{B} \cap[a c]$.

Notemos que a aplicação $I_{b}:[c] \rightarrow[b c]$ é mensurável com inversa mensurável, pois $I_{b}$ é a aplicação inversa de $\left.\sigma\right|_{[b c]}:[b c] \rightarrow[b]$ o qual é um homeomorfismo, assim para todo $E \in \mathcal{B} \cap[c]$ 
temos $I_{b}(E) \in \mathcal{B} \cap[b c]$. Seja $E \in \mathcal{B} \cap[c]$ tal que $\mu(E)=0$, pelo fato de $\mu$ ser não-singular temos $\mu\left(\sigma^{-1} E\right)=0$. Além disso $I_{b}(E) \subset \sigma^{-1} E$, $\log 0 \mu \circ I_{b}(E)=0$. Seja $E \in \mathcal{B} \cap[c]$ tal que $\mu \circ I_{b}(E)=0$, sabemos que $I_{b}(E) \in \mathcal{B} \cap[b c]$ e $\mu\left(I_{b}(E)\right)=0$, pelo item ii. $)$, temos $0=\mu\left(\left.\sigma\right|_{[b c]}\left(I_{b}(E)\right)\right)=\mu(E)$. Portanto $\mu \circ I_{b} \sim \mu$ em $\mathcal{B} \cap[c]$.

Suponhamos que $\mu([a c])=0$, para cada $b \in S$ tal que $A(b, c)=1$ temos $v_{a c, b c}[a c]=[b c]$. Assim pela Proposição 81 temos que $\mu([b c])=0$ para todo $b \in S$ tal que $A(b, c)=1$. Logo

$$
\mu\left(\left.\sigma^{-1} \sigma\right|_{[a c]}[a c]\right)=\mu\left(\bigcup_{A(b, c)=1}[b c]\right)=\sum_{A(b, c)=1} \mu([b c])=0 .
$$

Como $\mu$ é não-singular então $\mu([c])=\mu\left(\left.\sigma\right|_{[a c]}[a c]\right)=0$.

Proposição 88. Sejam $\Sigma_{A}$ um shift de Markov, $\phi: \Sigma_{A} \rightarrow \mathbb{R}$ um potencial mensurável e $\mu$ uma medida $\phi$-DLR não-singular. Então para cada $c \in S$ e cada $a, b \in S$ tais que $A(a, c)=A(b, c)=1$ e $\mu([a c])>0$, temos

$$
\frac{\frac{\mathrm{d} \mu}{\mathrm{d} \mu \odot \sigma}(a x)}{e^{\phi(a x)}}=\frac{\frac{\mathrm{d} \mu}{\mathrm{d} \mu \odot \sigma}(b x)}{e^{\phi(b x)}}, \quad \mu-\text { q.t.p. } e m[c] .
$$

Demonstração. Como $\mu$ é não-singular então pelo Lema 16 temos que $\mu \ll \mu \odot \sigma$, assim a derivada de Radon-Nikodým $\frac{\mathrm{d} \mu}{\mathrm{d} \mu \odot \sigma}$ está bem definida. Sejam $a, b \in S$ tais que $A(a, c)=A(b, c)=1$ e $\mu([a c])>0$. A aplicação $v_{a c, b c}:[a c] \mapsto[b c]$ pode-se escrever como

$$
v_{a c, b c}=\left.I_{b} \circ \sigma\right|_{[a c]},
$$

onde $I_{b}: x \mapsto(b, x)$. Pelo Lema 87 temos que $\mu \circ v_{a c, b c} \sim \mu$ e $\left.\mu \circ \sigma\right|_{[a c]} \sim \mu$ em $\mathcal{B} \cap[a c]$. Logo, para todo $y \in[a c]$, temos

$$
\begin{aligned}
\frac{\mathrm{d} \mu \circ v_{a c, b c}}{\mathrm{~d} \mu}(y) & =\frac{\left.\mathrm{d} \mu \circ I_{b} \circ \sigma\right|_{[a c]}}{\mathrm{d} \mu}(y) \\
& =\frac{\left.\mathrm{d} \mu \circ I_{b} \circ \sigma\right|_{[a c]}}{\left.\mathrm{d} \mu \circ \sigma\right|_{[a c]}}(y) \cdot \frac{\left.\mathrm{d} \mu \circ \sigma\right|_{[a c]}}{\mathrm{d} \mu}(y), \quad \mu-\text { q.t.p. em }[a c]
\end{aligned}
$$

é dizer, se

$$
E=\left\{y \in[a c] \mid \frac{\mathrm{d} \mu \circ v_{a c, b c}}{\mathrm{~d} \mu}(y) \neq \frac{\left.\mathrm{d} \mu \circ I_{b} \circ \sigma\right|_{[a c]}}{\left.\mathrm{d} \mu \circ \sigma\right|_{[a c]}}(y) \cdot \frac{\left.\mathrm{d} \mu \circ \sigma\right|_{[a c]}}{\mathrm{d} \mu}(y)\right\},
$$

então $\mu(E)=0$. Porém pelo Lema 87 item $i i$. $)$, temos $\mu\left(\left.\sigma\right|_{[a c]} E\right)=0$. Assim,

$$
\begin{aligned}
\left.\sigma\right|_{[a c]}(E) & =\left\{x \in[c] \mid \frac{\mathrm{d} \mu \circ v_{a c, b c}}{\mathrm{~d} \mu}\left(\left.\sigma\right|_{[a c]} ^{-1}(x)\right) \neq \frac{\left.\mathrm{d} \mu \circ I_{b} \circ \sigma\right|_{[a c]}}{\left.\mathrm{d} \mu \circ \sigma\right|_{[a c]}}\left(\left.\sigma\right|_{[a c]} ^{-1}(x)\right) \cdot \frac{\left.\mathrm{d} \mu \circ \sigma\right|_{[a c]}}{\mathrm{d} \mu}\left(\left.\sigma\right|_{[a c]} ^{-1}(x)\right)\right\} \\
& =\left\{x \in[c] \mid \frac{\mathrm{d} \mu \circ v_{a c, b c}}{\mathrm{~d} \mu}(a x) \neq \frac{\left.\mathrm{d} \mu \circ I_{b} \circ \sigma\right|_{[a c]}}{\left.\mathrm{d} \mu \circ \sigma\right|_{[a c]}}(a x) \cdot \frac{\left.\mathrm{d} \mu \circ \sigma\right|_{[a c]}}{\mathrm{d} \mu}(a x)\right\}
\end{aligned}
$$

tem medida zero, logo

$$
\frac{\mathrm{d} \mu \circ v_{a c, b c}}{\mathrm{~d} \mu}(a x)=\frac{\left.\mathrm{d} \mu \circ I_{b} \circ \sigma\right|_{[a c]}}{\left.\mathrm{d} \mu \circ \sigma\right|_{[a c]}}(a x) \cdot \frac{\left.\mathrm{d} \mu \circ \sigma\right|_{[a c]}}{\mathrm{d} \mu}(a x), \quad \mu \text { - q.t.p. em }[c] .
$$

Como $\mu \circ I_{b} \sim \mu$ em $\mathcal{B} \cap[c]$ e $\left.\sigma\right|_{[a c]}$ é uma aplicação mensurável e invertível, pelo Lema 83 item ii.) 
temos que para cada $y \in[a c]$ segue

$$
\frac{\left.\mathrm{d} \mu \circ I_{b} \circ \sigma\right|_{[a c]}}{\left.\mathrm{d} \mu \circ \sigma\right|_{[a c]}}(y)=\frac{\mathrm{d} \mu \circ I_{b}}{\mathrm{~d} \mu} \circ \sigma(y)=\frac{\mathrm{d} \mu \circ I_{b}}{\mathrm{~d} \mu}(\sigma y), \quad \mu \circ \sigma-\text { q.t.p. em }[a c] .
$$

Pelo mesmo argumento para derivar a Equação (2.44), obtemos

$$
\frac{\left.\mathrm{d} \mu \circ I_{b} \circ \sigma\right|_{[a c]}}{\left.\mathrm{d} \mu \circ \sigma\right|_{[a c]}}(a x)=\frac{\mathrm{d} \mu \circ I_{b}}{\mathrm{~d} \mu}(x), \quad \mu-\text { q.t.p. em }[c] .
$$

Substituindo a Equação (2.45) em (2.44)

$$
\frac{\mathrm{d} \mu \circ v_{a c, b c}}{\mathrm{~d} \mu}(a x)=\frac{\mathrm{d} \mu \circ I_{b}}{\mathrm{~d} \mu}(x) \cdot \frac{\left.\mathrm{d} \mu \odot \sigma\right|_{[a c]}}{\mathrm{d} \mu}(a x), \quad \mu-\text { q.t.p. em }[c] .
$$

A seguir calcularemos cada derivada de Radon-Nikodým do lado direito da Equação (2.46).

i.) Como $I_{b}$ é uma aplicação mensurável, invertível, $\left.\sigma\right|_{[b c]} \circ I_{b}=\mathrm{id}$ e $\left.\mu \circ \sigma\right|_{[b c]}=\left.\mu \odot \sigma\right|_{[b c]}$ em $[b c]$, temos

$$
\begin{aligned}
\frac{\mathrm{d} \mu \circ I_{b}}{\mathrm{~d} \mu}(x) & =\frac{\mathrm{d} \mu \circ I_{b}}{\left.\mathrm{~d} \mu \circ \sigma\right|_{[b c]} \circ I_{b}}(x)=\frac{\mathrm{d} \mu}{\left.\mathrm{d} \mu \circ \sigma\right|_{[b c]}} \circ I_{b}(x) \\
& =\frac{\mathrm{d} \mu}{\left.\mathrm{d} \mu \circ \sigma\right|_{[b c]}}(b x)=\frac{\mathrm{d} \mu}{\left.\mathrm{d} \mu \odot \sigma\right|_{[b c]}}(b x),\left.\quad \mu \circ \sigma\right|_{[b c]} \circ I_{b}-\text { q.t.p. em }[c]
\end{aligned}
$$

Assim, do fato que $\left.\sigma\right|_{[b c]} \circ I_{b}=\mathrm{id}$, temos

$$
\frac{\mathrm{d} \mu \circ I_{b}}{\mathrm{~d} \mu}(x)=\frac{\mathrm{d} \mu}{\left.\mathrm{d} \mu \odot \sigma\right|_{[b c]}}(b x), \quad \mu \text { - q.t.p. em }[c] .
$$

ii.) Como $\left.\mu \odot \sigma\right|_{[a c]} \sim \mu \mathrm{em} \mathcal{B} \cap[a c]$, temos

$$
\frac{\left.\mathrm{d} \mu \odot \sigma\right|_{[a c]}}{\mathrm{d} \mu}(a x)=\left(\frac{\mathrm{d} \mu}{\left.\mathrm{d} \mu \odot \sigma\right|_{[a c]}}\right)^{-1}(a x)=\frac{1}{\frac{\mathrm{d} \mu}{\left.\mathrm{d} \mu \odot \sigma\right|_{[a c]}}(a x)}, \quad \mu-\text { q.t.p. em }[c] .
$$

Substituindo as Equações (2.47) e (2.48) em (2.46),

$$
\frac{\mathrm{d} \mu \circ v_{a c, b c}}{\mathrm{~d} \mu}(a x)=\frac{\frac{\mathrm{d} \mu}{\mathrm{d} \mu \odot \sigma}(b x)}{\frac{\mathrm{d} \mu}{\mathrm{d} \mu \odot \sigma}(a x)}, \quad \mu \text { - q.t.p. em }[c] .
$$

Considere

$$
F=\left\{x \in[a c] \mid \frac{\mathrm{d} \mu \circ v_{a c, b c}}{\mathrm{~d} \mu}(x) \neq \frac{e^{\phi_{2}\left(b c x_{2}^{\infty}\right)}}{e^{\phi_{2}\left(a c x_{2}^{\infty}\right)}}\right\}
$$

pela Proposição 81 temos que $\mu(F)=0$. Pelo Lema 87 item ii.) temos $\mu\left(\left.\sigma\right|_{[a c]}(F)\right)=0$, é dizer,

$$
\begin{aligned}
\left.\sigma\right|_{[a c]}(F) & =\left\{x \in[c] \mid \frac{\mathrm{d} \mu \circ v_{a c, b c}}{\mathrm{~d} \mu}\left(\left.\sigma\right|_{[a c]} ^{-1}(x)\right) \neq \frac{e^{\phi_{2}\left(\left.b c \sigma^{2} \sigma\right|_{[a c]} ^{-1}(x)\right)}}{e^{\phi_{2}\left(\left.a c \sigma^{2} \sigma\right|_{[a c]} ^{-1}(x)\right)}}\right\} \\
& =\left\{x \in[c] \mid \frac{\mathrm{d} \mu \circ v_{a c, b c}}{\mathrm{~d} \mu}(a x) \neq \frac{e^{\phi_{2}(b x)}}{e^{\phi_{2}(a x)}}\right\}
\end{aligned}
$$


tem medida zero. Logo

$$
\frac{\mathrm{d} \mu \circ v_{a c, b c}}{\mathrm{~d} \mu}(a x)=\frac{e^{\phi(b x)}}{e^{\phi(a x)}}, \quad \mu-\text { q.t.p. em }[c] .
$$

Finalmente das Equações (2.49) e (2.50) obtemos a Equação (2.43).

Teorema 89. Sejam $\Sigma_{A}$ um shift de Markov, $\phi: \Sigma_{A} \rightarrow \mathbb{R}$ um potencial mensurável e $m$ uma medida $\phi$-DLR $\sigma$-invariante. Então $L_{\phi}(\mathbb{1})=\lambda m$-q.t.p. se, e somente, se $m$ é $(\phi, \lambda)$-conforme, onde $\lambda>0$.

Demonstração. Pelo Corolário 23, temos

$$
1=\sum_{\sigma y=x} \frac{\mathrm{d} m}{\mathrm{~d} m \odot \sigma}(y), \quad m-\text { q.t.p. em } \Sigma_{A} .
$$

Fixado $c \in S$ tal que $m([c])>0$. Da Equação (2.51) obtemos

$$
1=\sum_{b \in S \mid A(b, c)=1} \frac{\mathrm{d} m}{\mathrm{~d} m \odot \sigma}(b x), \quad m \text { - q.t.p. em }[c] .
$$

Como $m\left(\sigma^{-1}[c]\right)>0$, fixemos $a \in S$ tais que $A(a, c)=1$ e $m([a c])>0$. Pela Proposição 88, para cada $b \in S$ tal que $A(b, c)=1$ temos

$$
\frac{\mathrm{d} m}{\mathrm{~d} m \odot \sigma}(b x)=e^{\phi(b x)} \frac{\frac{\mathrm{d} m}{\mathrm{~d} m \odot \sigma}(a x)}{e^{\phi(a x)}}, \quad m-\text { q.t.p. em }[c],
$$

definamos

$$
E_{b}:=\left\{x \in[c] \mid \frac{\mathrm{d} m}{\mathrm{~d} m \odot \sigma}(b x)=e^{\phi(b x)} \frac{\frac{\mathrm{d} m}{\mathrm{~d} m \odot \sigma}(a x)}{e^{\phi(a x)}}\right\},
$$

da Equação (2.53) obtemos que $m\left(E_{b}\right)=m([c])$ para todo $b \in S$ tal que $A(b, c)=1$. Consideremos $E:=\cap_{b \in S \mid A(b, c)=1} E_{b}$, então $m(E)=m([c]) \mathrm{e}$

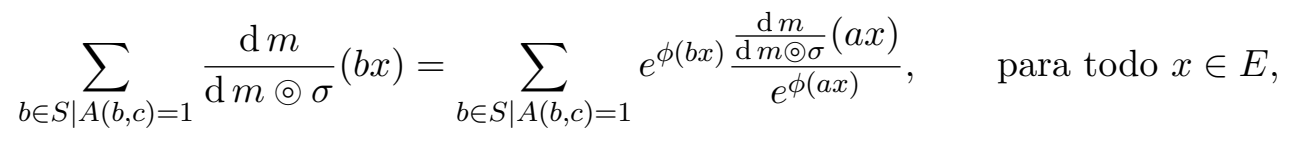

portanto

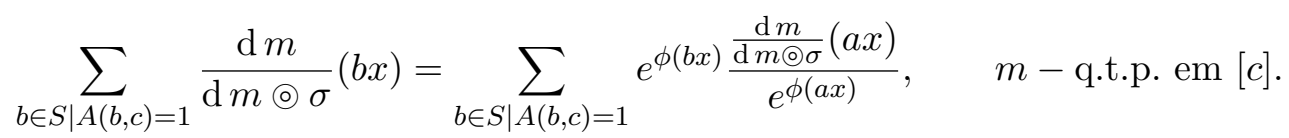

Substituindo (2.54) na Equação (2.52) obtemos

$$
\begin{aligned}
1 & =\sum_{b \in S \mid A(b, c)=1} e^{\phi(b x)} \frac{\frac{\mathrm{d} m}{\mathrm{~d} m \odot \sigma}(a x)}{e^{\phi(a x)}} \\
& =\frac{\frac{\mathrm{d} m}{\mathrm{~d} m \odot \sigma}(a x)}{e^{\phi(a x)}} \sum_{b \in S \mid A(b, c)=1} e^{\phi(b x)} \\
& =\frac{\frac{\mathrm{d} m}{\mathrm{~d} m \odot \sigma}(a x)}{e^{\phi(a x)}} \sum_{\sigma y=x} e^{\phi(y)}, \quad m-\text { q.t.p. em }[c] .
\end{aligned}
$$


Seja $\lambda>0$ tal que $L_{\phi} \mathbb{1}=\lambda$. Da Equação (2.55) temos

$$
\frac{\mathrm{d} m}{\mathrm{~d} m \odot \sigma}(a x)=\lambda^{-1} e^{\phi(a x)}, \quad m \text { - q.t.p. em }[c] .
$$

Pelo item ii.) do Lema 87 obtemos que a Equação (2.56) é $m$ - q.t.p. em [ac], é dizer,

$$
m(F)=\lambda^{-1} \int_{F} e^{\phi(x)} \mathrm{d} m \odot \sigma, \quad \forall F \in \mathcal{B} \cap[a c] .
$$

Note que para todo $\omega \in S$ tal que $A(\omega, c)=1$ e $m([\omega c])=0$ temos que

$$
m \odot \sigma[\omega c]=0,
$$

pois pelo Lema $87 m([c])=0$ e $m \odot \sigma([\omega c])=m([c])$.

Seja $\mathcal{W}_{2}(c)=\left\{\omega \in \mathcal{W}_{2} \mid \omega_{1}=c\right\}$. Cada $G \in \mathcal{B}$ pode-se escrever como $G=\bigsqcup_{c \in S} \bigsqcup_{\omega \in \mathcal{W}_{2}(c)} G \cap[\omega]$, além disso pelas Equações (2.57) e (2.58) obtemos

$$
\begin{aligned}
m(G) & =\sum_{c \in S} \sum_{\omega \in \mathcal{W}_{2}(c)} m(G \cap[\omega])=\sum_{\substack { c \in S \\
\begin{subarray}{c}{\omega \in \mathcal{W}_{2}(c) \\
m([\omega])>0{ c \in S \\
\begin{subarray} { c } { \omega \in \mathcal { W } _ { 2 } ( c ) \\
m ( [ \omega ] ) > 0 } }\end{subarray}} m(G \cap[\omega]) \\
& =\sum_{c \in S} \sum_{\substack{\omega \in \mathcal{W}_{2}(c) \\
m([\omega])>0}} \lambda^{-1} \int_{G \cap[\omega]} e^{\phi(x)} \mathrm{d} m \odot \sigma \\
& =\lambda^{-1} \sum_{c \in S} \sum_{\omega \in \mathcal{W}_{2}(c)} \int_{G \cap[\omega]} e^{\phi(x)} \mathrm{d} m \odot \sigma \\
& =\lambda^{-1} \sum_{c \in S} \int_{G \cap \pi_{1}^{-1}\{c\}} e^{\phi(x)} \mathrm{d} m \odot \sigma \\
& =\lambda^{-1} \int_{G} e^{\phi(x)} \mathrm{d} m \odot \sigma
\end{aligned}
$$

portanto $m$ é uma medida $(\phi, \lambda)$-conforme.

Consideremos $m$ uma medida $(\phi, \lambda)$-conforme. Da Equação (2.55) temos que para cada $c \in S$ tal que $m([c])>0$,

$$
\sum_{\sigma y=x} e^{\phi(y)}=\lambda, \quad m \text { - q.t.p. em }[c] .
$$

Portanto $L_{\phi} \mathbb{1}=\lambda, m-$ q.t.p..

A seguir temos um shift de Markov, um potencial e uma medida DLR satisfazendo as hipóteses do Teorema acima.

Exemplo 90. Consideremos $\Sigma_{A}$ o shift de Markov dado pelo grafo da Figura 2.1 e $\phi: \Sigma_{A} \rightarrow$ $\{\log \lambda\}$, onde $\lambda>0$. Como $\phi=\log \lambda$ e para cada $x \in \Sigma_{A}$ só existe uma pré-imagem então

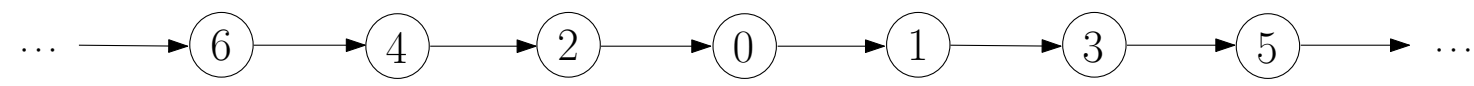

Figura 2.1: shift linha. 
$L_{\phi} \mathbb{1}=\lambda$. Sejam

$$
x_{i}= \begin{cases}(i, i+2, i+4 \ldots), & \text { se } i \text { é } \text { impar } ; \\ (0,1,3,5, \ldots), & \text { se } i=0 \\ (i, i-2, \ldots, i-(i-2 k), 0,1, \ldots), & \text { se } i=2 k, k \text { natural }\end{cases}
$$

e $m$ uma medida definida por

$$
m(E):=\sum_{i \geq 0} \delta_{x_{i}}(E) \quad \text { para todo } E \in \mathcal{B} .
$$

A medida $m$ tem as seguintes propriedades:

i.) $m$ é $\sigma$-finita e $m\left(\Sigma_{A}\right)=+\infty$. Basta notar que $m([\omega])=1$ para todo cilindro $\omega$ de tamanho arbitrário.

ii.) $m$ é uma medida $\phi$ - DLR. De fato, fixado $n \in \mathbb{N}$, seja $a:=\left(a_{0}, a_{1}, \ldots, a_{n-1}\right)$ uma palavra permitida de tamanho n e $E \in \sigma^{-n} \mathcal{B}$, logo:

$$
\begin{aligned}
\int_{E} \frac{e^{\phi_{n}\left(a \sigma^{n} x\right)}}{\sum_{\sigma^{n} y=\sigma^{n} x} e_{\left\{a \sigma^{n} x \in \Sigma_{A}\right\}}} \mathrm{d} m(x) & =\int \mathbb{1}_{E}(x) \mathbb{1}_{\left\{a \sigma^{n} x \in \Sigma_{A}\right\}} \mathrm{d} m(x) \\
& =\sum_{i \geq 0} \mathbb{1}_{E}\left(x_{i}\right) \mathbb{1}_{\left\{a \sigma^{n} x_{i} \in \Sigma_{A}\right\}} \\
& =\mathbb{1}_{E}\left(x_{a_{0}}\right),
\end{aligned}
$$

$e$

$$
\begin{aligned}
\int_{E} \mathbb{E}_{m}\left[\mathbb{1}_{[a]} \mid \sigma^{-n} \mathcal{B}\right](x) \mathrm{d} m(x) & =\int \mathbb{1}_{E \cap[a]}(x) \mathrm{d} m(x) \\
& =\sum_{i \geq 0} \mathbb{1}_{E}\left(x_{i}\right) \mathbb{1}_{[a]}\left(x_{i}\right) \\
& =\mathbb{1}_{E}\left(x_{a_{0}}\right) .
\end{aligned}
$$

De (2.59) e (2.60) temos que m é uma medida $\phi$ - DLR.

iii.) $m$ é $\sigma$-invariante. Seja $\mathcal{C}$ a álgebra gerada pelos cilindros de $\Sigma_{A}$. Para cada $\omega \in \mathcal{C}$ temos que $m(\omega)=m \circ \sigma^{-1}(\omega)$, logo $m(E)=m \circ \sigma^{-1}(E)$ para todo $E \in \mathcal{B}$. Assim, $m$ é $\sigma$-invariante.

Notemos que $m(\omega)=m \odot \sigma(\omega)$ para todo $\omega \in \mathcal{C}$ logo $m$ é $(\phi, \lambda)$-conforme.

\subsection{Equivalência entre Medidas DLR e de Equilíbrio}

Nesta seção estudaremos as relações das medidas de equilíbrio e as medidas DLR para o espaço shift de Markov com alfabeto enumerável. Esse problema já foi abordado por G. Keller, B. Kimura e S. Muir nos trabalhos [Kel, Ki, Mul] respectivamente, entre outros, neste caso o espaço é dado pelo full shift em $S^{\mathbb{Z}^{d}}$, onde $S$ é um alfabeto enumerável, e mostram que ambos conceitos coincidem assumindo alguma regularidade no potencial.

O. Sarig mostrou a equivalência entre medidas DLR e medidas conformes para o caso de shift de Markov topologicamente mixing com alfabeto finito, isto é o caso compacto. 
Teorema 91 ([Sa5]). Sejam $\Sigma_{A}$ o espaço shift de Markov topologicamente mixing com alfabeto finito e $\phi: \Sigma_{A} \rightarrow \mathbb{R}$ um potencial satisfazendo a condição de Walters. Existe uma única medida $\phi-\mathrm{DLR}$ e esta é conforme.

Demonstração. Para os detalhes da prova, veja Teorema 3.6 em [Sa5].

L. Cioletti e A. Lopes mostram o mesmo resultado para o caso do full shift através de uma prova alternativa, veja [CiLo]. Nesta seção mostraremos que esta equivalência segue sendo válida para o caso BIP com alfabeto enumerável, Teorema 93, e mostraremos as relações entre medidas de equilíbrio e DLR, veja o Teorema 96.

O seguinte lema será importante na prova dos próximos resultados.

Lema 92. Sejam $(\Omega, \mathcal{F})$ um espaço mensurável, $f: \Omega \rightarrow \mathbb{R} e\left\{F_{i}\right\}_{i \geq 1}$ uma partição $\mathcal{F}$-mensurável de $\Omega$. Então $f$ é $\mathcal{F}$-mensurável se, e somente, se para cada $i \geq 1$ temos que $\left.f\right|_{F_{i}}: \Omega \rightarrow \mathbb{R}$ é $\left.\mathcal{F}\right|_{F_{i}}-$ mensurável.

A seguir discutiremos hipóteses no shift de Markov e no potencial para que a medida DLR exista e seja única. Notemos que este teorema mostra a equivalência entre as medidas DLR e conformes.

Teorema 93. Sejam $\Sigma_{A}$ um shift de Markov topologicamente mixing com a propriedade BIP $e$ $\phi: \Sigma_{A} \rightarrow \mathbb{R}$ um potencial que satisfaz a condição de Walters com $\operatorname{Var}_{1} \phi<\infty$ e $P_{G}(\phi)<\infty$. Então existe uma única medida DLR e esta é de probabilidade. Em particular as medidas DLR $e$ conformes de probabilidade coincidem.

Demonstração. Pelas hipóteses do teorema temos que $\phi$ é positivamente recorrente. Logo existem uma medida $\nu \in \mathcal{M}^{1}\left(\Sigma_{A}\right)$ e uma função contínua $h: \Sigma_{A} \rightarrow \mathbb{R}$ afastada de zero e do infinito tais que $L_{\phi} h=\lambda h$ e $L_{\phi}^{*} \nu=\lambda \nu$, onde $\lambda=e^{P_{G}(\phi)}$, veja [Sa4]. A prova será feita em dois passos, primeiro mostraremos que $\mu \ll \nu$, onde $\mu$ é uma medida DLR, logo existe uma função mensurável $\varphi=\frac{\mathrm{d} \mu}{\mathrm{d} \nu}$ e finalmente mostraremos que $\varphi$ é constante igual a $1 \nu$ - q.t.p..

Afirmação 3. $\mu$ é absolutamente contínua em relação a $\nu$.

Sejam $n \in \mathbb{N}$ e $[\underline{a}]$ um cilindro de tamanho $n$. Denotemos $M:=\sup _{n \geq 1} \operatorname{Var}_{n+1} \phi_{n}+\operatorname{Var}_{1} \phi$, então $\left|\phi_{n}(\xi)-\phi_{n}(\eta)\right| \leq M$ para todo $\xi, \eta \in[\underline{a}]$. Fixado $x \in \Sigma_{A}$, temos

$$
e^{\phi_{n}\left(\underline{a} \sigma^{n} x\right)} \mathbb{1}_{\left\{\underline{a} \sigma^{n} x \in \Sigma_{A}\right\}} \leq e^{M} e^{\phi_{n}(\underline{a} y)}, \quad \forall y \in \sigma^{n}[\underline{a}] .
$$

Integrando em relação a medida $\nu$ temos

$\int_{\sigma^{n}[\underline{a}]} e^{\phi_{n}\left(\underline{a} \sigma^{n} x\right)} \mathbb{1}_{\left\{\underline{a} \sigma^{n} x \in \Sigma_{A}\right\}} \mathrm{d} \nu(y) \leq e^{M} \int_{\sigma^{n}[\underline{a}]} L_{\phi}^{n} \mathbb{1}_{[\underline{a}]}(y) \mathrm{d} \nu(y) \leq e^{M} \int_{\Sigma_{A}} L_{\phi}^{n} \mathbb{1}_{[\underline{a}]}(y) \mathrm{d} \nu(y)=e^{M} \lambda^{n} \nu([\underline{a}])$.

Portanto

$$
\lambda^{-n} e^{\phi_{n}\left(\underline{a} \sigma^{n} x\right)} \mathbb{1}_{\left\{\underline{a} \sigma^{n} x \in \Sigma_{A}\right\}} \leq \frac{1}{\nu\left(\sigma^{n}[\underline{a}]\right)} e^{M} \nu([\underline{a}]), \quad \forall x \in \Sigma_{A} .
$$

Sejam $b_{1}, b_{2}, \cdots, b_{N} \in S$ como na definição da propriedade $B I P$. Logo existe $i \in\{1,2, \cdots, N\}$ tal que $A\left(a_{n-1}, b_{i}\right)=1$, ou seja, $\sigma^{n}[\underline{a}] \supset\left[b_{i}\right]$, assim obtemos

$$
\nu\left(\sigma^{n}[\underline{a}]\right) \geq \min \left\{\nu\left(\left[b_{i}\right]\right) \mid i=1, \ldots, N\right\}:=K>0 .
$$


Note que $K$ é positivo pois a medida $\nu$ em cilindros é positiva. Substituindo na Equação (2.61) obtemos

$$
\lambda^{-n} e^{\phi_{n}\left(\underline{a} \sigma^{n} x\right)} \mathbb{1}_{\left\{\underline{a} \sigma^{n} x \in \Sigma_{A}\right\}} \leq \frac{e^{M}}{K} \nu([\underline{a}]), \quad \forall x \in \Sigma_{A} .
$$

Pelo fato de $\phi$ ser positivamente recorrente e $\Sigma_{A}$ ter a propriedade $B I P$, existem constantes positivas $H_{1}$ e $H_{2}$ tais que $H_{1} \leq h \leq H_{2}$ e além disso $L_{\phi}^{n} h(\xi)=\lambda^{n} h(\xi)$ para todo $n \in \mathbb{N}$ e $\xi \in \Sigma_{A}$, $\log 0$

$$
\lambda^{n} H_{1} \leq \lambda^{n} h\left(\sigma^{n} x\right)=\sum_{\sigma^{n} y=\sigma^{n} x} e^{\phi_{n}(y)} h(y) \leq H_{2} \sum_{\sigma^{n} y=\sigma^{n} x} e^{\phi_{n}(y)},
$$

$\operatorname{assim}$ obtemos $\frac{H_{1}}{H_{2}} \leq \lambda^{-n} \sum_{\sigma^{n} y=\sigma^{n} x} e^{\phi_{n}(y)}$. Analogamente $\lambda^{-n} \sum_{\sigma^{n} y=\sigma^{n} x} e^{\phi_{n}(y)} \leq \frac{H_{2}}{H_{1}}$. Portanto

$$
\frac{H_{1}}{H_{2}} \leq \lambda^{-n} \sum_{\sigma^{n} y=\sigma^{n} x} e^{\phi_{n}(y)} \leq \frac{H_{2}}{H_{1}}
$$

para todo $x \in \Sigma_{A}$. Das Equações (2.63) e (2.64) obtemos que dado $n \in \mathbb{N}$, e $[\underline{a}]$ um cilindro de tamanho $n$ então

$$
\frac{e^{\phi_{n}\left(\underline{a} \sigma^{n} x\right)} \mathbb{1}_{\left\{\underline{a} \sigma^{n} x \in \Sigma_{A}\right\}}}{\sum_{\sigma^{n} y=\sigma^{n} x} e^{\phi_{n}(y)}} \leq \frac{H_{1} e^{M}}{H_{2} K} \nu([\underline{a}]), \quad \forall x \in \Sigma_{A},
$$

desde que $\mu$ é uma medida $\phi$-DLR obtemos que $\mathbb{E}_{\mu}\left[\mathbb{1}_{[\underline{a}]} \mid \sigma^{-n} \mathcal{B}\right](x) \leq C \nu[\underline{a}]$, onde $C=\frac{H_{1} e^{M}}{H_{2} K}$. Portanto $\mu[\underline{a}] \leq C \nu[\underline{a}]$ para todo cilindro de tamanho $n$, desde que $n$ é arbitrário obtemos que $\mu \leq C \nu$ é satisfeito para qualquer cilindro, $\log 0 \mu \ll \nu$ e a medida $\phi-$ DLR é de probabilidade. $\triangle$

Desde que $\mu \ll \nu$ então existe uma função mensurável $\varphi: \Sigma_{A} \rightarrow[0, \infty)$ tal que $\varphi=\frac{\mathrm{d} \mu}{\mathrm{d} \nu}$.

Afirmação 4. $\varphi=1 \nu$ - q.t.p..

Fixado $n \geq 2$, sejam $[\underline{a}]:=\left[a_{0}, a_{1}, \ldots, a_{n-1}\right]$ e $[\underline{b}]:=\left[b_{0}, b_{1}, \ldots, b_{n-1}\right]$ dois cilindros de tamanho $n$ tais que $a_{n-1}=b_{n-1}$. Desde que $\mu$ e $\nu$ são $\phi-D L R$, temos que

$$
\left.\frac{\mathrm{d} \mu \circ v_{\underline{a}, \underline{b}}}{\mathrm{~d} \mu}\right|_{[\underline{a}]}=e^{\phi_{n}\left(\underline{b} x_{n}^{\infty}\right)-\phi_{n}\left(\underline{a} x_{n}^{\infty}\right)}, \quad \mu \text { - q.t.p. em }[\underline{a}],
$$

e

$$
\left.\frac{\mathrm{d} \nu \circ v_{\underline{a}, \underline{b}}}{\mathrm{~d} \nu}\right|_{[\underline{a}]}=e^{\phi_{n}\left(\underline{b} x_{n}^{\infty}\right)-\phi_{n}\left(\underline{a} x_{n}^{\infty}\right)}, \quad \nu \text { - q.t.p. em }[\underline{a}] .
$$

Das Equações (2.65), (2.66) e $\mu \ll \nu$ temos que $\left.\frac{\mathrm{d} \mu \circ v_{\underline{a}, \underline{b}}}{\mathrm{~d} \mu}\right|_{[\underline{a]}}=\left.\frac{\mathrm{d} \nu \circ v_{\underline{a}, \underline{b}}}{\mathrm{~d} \nu}\right|_{[\underline{a}]} \mu-$ q.t.p., isto é,

$$
\left(\mu \circ v_{\underline{a}, \underline{b}}\right)(E)=\int_{E} \frac{\mathrm{d} \nu \circ v_{\underline{a}, \underline{b}}}{\mathrm{~d} \nu} \mathrm{d} \mu
$$

para todo $E \in \mathcal{B} \cap[\underline{a}]$. Notemos que $v_{\underline{a}, \underline{b}}$ é uma aplicação bijetora e contínua, para $F \in \mathcal{B} \cap[\underline{b}]$ temos que $G:=v_{\underline{a}, \underline{b}}^{-1}(F) \in \mathcal{B} \cap[\underline{a}]$, logo usando a Equação (2.67) temos

$$
\begin{aligned}
\int_{F} \varphi \mathrm{d} \nu=\int_{v_{\underline{a}, \underline{b}}(G)} \mathrm{d} \mu & =\mu \circ v_{\underline{a}, \underline{b}}(G) \\
& =\int_{G} \frac{\mathrm{d} \nu \circ v_{\underline{a}, \underline{b}}}{\mathrm{~d} \nu} \mathrm{d} \mu \\
& =\int_{G} \varphi \mathrm{d} \nu \circ v_{\underline{a}, \underline{b}}
\end{aligned}
$$




$$
=\int_{F} \varphi \circ v_{\underline{b}, \underline{a}} \mathrm{~d} \nu
$$

Desde que $F \subset \mathcal{B} \cap[\underline{b}]$ é arbitrário temos que $\varphi(\underline{a} x)=\varphi(\underline{b} x), \nu$ - q.t.p., onde $\underline{a}$ e $\underline{b}$ são palavras arbitrárias de tamanho $n \in \mathbb{N}$ tal que $a_{n-1}=b_{n-1}$.

Fixemos $c \in S$ logo para cada $\underline{w_{1}}, \underline{w_{2}} \in \mathcal{W}_{n}(c)$ temos que $\varphi\left(\underline{w_{1}} x_{n}^{\infty}\right)=\varphi\left(\underline{w_{2}} x_{n}^{\infty}\right)$, é dizer $\left.\varphi\right|_{\pi_{n}^{-1}\{c\}}$ só depende das coordenadas $x_{k}$, para $k \geq n$, logo é $\left.\sigma^{-n} \mathcal{B}\right|_{\pi_{n}^{-1}\{c\}}$-mensurável, desde que $c \in S$ é arbitrário pelo Lema 92 temos que $\varphi$ é $\sigma^{-n} \mathcal{B}$-mensurável. Como $n \geq 2$ é arbitrário temos que $\varphi$ é $\sigma^{-n} \mathcal{B}$-mensurável para cada $n \in \mathbb{N} \operatorname{logo} \varphi$ é mensurável em relação a $\sigma$-álgebra caudal $\cap_{n \geq 1} \sigma^{-n} \mathcal{B}$.

Como $h \mathrm{~d} \nu$ é uma medida $\sigma$-invariante $\log o \nu$ é não-singular. Pelo Teorema 2.5 em [Sa5] temos que $\nu$ é exata, $\log 0 \varphi$ é constante $\nu$ - q.t.p.. Pelo fato de $\mu$ e $\nu$ ser medidas de probabilidade temos que $\varphi=1 \nu$ - q.t.p..

Como consequência da Afirmação 4 temos que $\nu=\mu$.

A seguir expomos um shift topologicamente mixing que na ausência da condição $B I P$ existe uma medida DLR que não é $(\phi, \lambda)$-conforme, para qualquer $\lambda>0$. Este exemplo foi inspirado em um shift de Markov dado por O. Shwartz, veja o Exemplo 2 em [Sw].

Exemplo 94. Consideremos o shift de Markov definido pelo grafo da Figura 2.2 e $\phi: \Sigma_{A} \rightarrow \mathbb{R}$ um potencial arbitrário (em particular pode ser um potencial satisfazendo a condição de Walters tais que $\operatorname{Var}_{1}<\infty$ e $\left.P_{G}(\phi)<\infty\right)$. Notemos que $\Sigma_{A}$ não satisfaz a propriedade BIP.

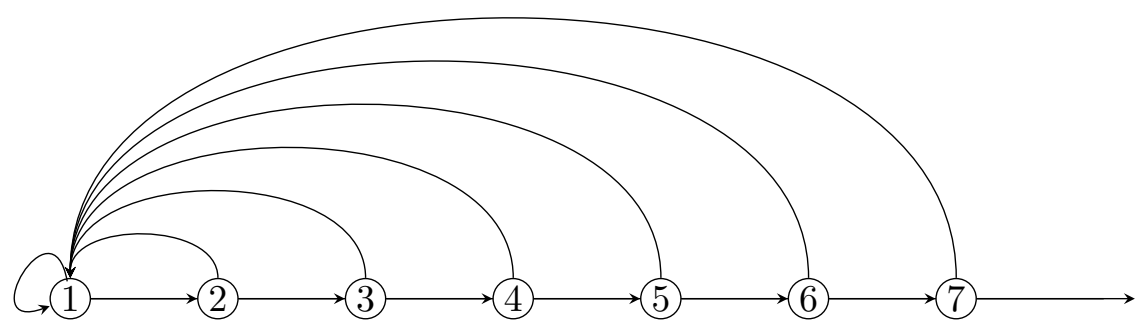

Figura 2.2

Seja $\bar{x}=(1,2, \ldots) \in \Sigma_{A}$. Definamos a medida de probabilidade $\mu:=\delta_{\bar{x}}$, afirmamos que $\mu$ é uma $\phi$ - DLR. Em efeito, $\mu$ é $\phi-\mathrm{DLR}$ se, e somente, se para cada $n \in \mathbb{N}$ e $\underline{a} \in \mathcal{W}_{n}$, temos

$$
\mathbb{E}\left[\mathbb{1}_{[\underline{a}]} \mid \sigma^{-n} \mathcal{B}\right](x)=\frac{e^{\phi_{n}\left(\underline{a} \sigma^{n} x\right)} \mathbb{1}_{\left\{\underline{a} \sigma^{n} x \in \Sigma_{A}\right\}}}{\sum_{\sigma^{n} y=\sigma^{n} x} e^{\phi_{n}(y)}} ; \quad \mu \text { - q.t.p. }
$$

ou equivalentemente

$$
\int_{B} \mathbb{1}_{[\underline{a}]}(x) \mathrm{d} \mu=\int_{B} \frac{e^{\phi_{n}\left(\underline{a} \sigma^{n} x\right)} \mathbb{1}_{\left\{\underline{a} \sigma^{n} x \in \Sigma_{A}\right\}}}{\sum_{\sigma^{n} y=\sigma^{n} x} e^{\phi_{n}(y)}} \mathrm{d} \mu,
$$

para cada $B \in \sigma^{-n} \mathcal{B}$. Consideremos $\underline{a} \neq(1,2, \ldots, n)$, a Equação (2.68) é satisfeita. Suponhamos que $\underline{a}=(1,2, \ldots, n), \operatorname{logo}:$

$$
\text { - } \int_{B} \mathbb{1}_{[\underline{a}]}(x) \mathrm{d} \mu=\mathbb{1}_{B}(\bar{x})
$$


- $\int_{B} \frac{e^{\phi_{n}\left(\underline{a} \sigma^{n} x\right)}}{\sum_{\sigma^{n} y=\sigma^{n} x} e^{\phi_{n}(y)}} \mathrm{d} \mu=\mathbb{1}_{B}(\bar{x})$.

Assim temos que a Equação (2.68) é satisfeita, portanto $\mu$ é uma medida $\phi$ - DLR.

Como $\mu\left(\sigma^{-1}[1]\right)=0$ e $\mu \odot \sigma\left(\sigma^{-1}[1]\right)>0$, logo não existe $\lambda>0$ tal que $\mu$ seja $(\phi, \lambda)$-conforme, pois

$$
\mu\left(\sigma^{-1}[1]\right) \neq \lambda^{-1} \int_{\sigma^{-1}[1]} e^{\phi} \mathrm{d} \mu \odot \sigma
$$

para todo $\lambda>0$.

A seguir demonstraremos uma proposição que será importante para a discussão de quando uma medida $\phi$-equilíbrio é $\phi-$ DLR.

Proposição 95. Sejam $\Sigma_{A}$ um shift topologicamente mixing e $\phi: \Sigma_{A} \rightarrow \mathbb{R}$ um potencial recorrente que satisfaz a condição de Walters com $P_{G}(\phi)<\infty$. Consideremos $m:=h \mathrm{~d} \nu$ onde $h$ e $\nu$ é a autofunção e automedida do operador de Ruelle respectivamente, logo as seguintes afirmações são equivalentes:

i.) $m$ é $\phi-\mathrm{DLR}$;

ii.) $h(x)=\alpha, \nu$ - q.t.p., para algum $\alpha>0$.

Demonstração. Seja o potencial

$$
\widetilde{\phi}:=\phi+\log h-\log h \circ \sigma-P_{G}(\phi),
$$

que normaliza o operador de Ruelle, pode-se verificar que

$$
\int f \mathrm{~d} m=\int L_{\widetilde{\phi}} f \mathrm{~d} m, \quad \text { para toda } f \in L^{1}(m),
$$

veja a prova da Proposição $86, \operatorname{logo} m$ é uma medida $(\widetilde{\phi}, 1)$-conforme, quando $\phi$ é positivamente recorrente $m\left(\Sigma_{A}\right)$ é finita, e infinita para o caso nulamente recorrente. Como $\left\|L_{\widetilde{\phi}} \mathbb{1}\right\|_{\infty}<\infty$ então pelo Corolário 85 temos que $m$ é uma medida $\widetilde{\phi}-$ DLR e isso não depende da finitude de $m$.

A seguir mostraremos $i$.) $\Rightarrow$ ii.). Para cada $n \geq 2$ fixemos $c \in S, \underline{a}:=a_{0}, a_{1}, \ldots, a_{n-1}$ e $\underline{b}:=b_{0}, b_{1}, \ldots, b_{n-1}$ palavras de tamanho $n$ tal que $a_{n-1}=b_{n-1}=c$. Como $m$ é uma medida $\phi-$ DLR e $\tilde{\phi}-$ DLR pela Proposição 81 temos o seguinte

$$
\begin{aligned}
& m \circ v_{\underline{a}, \underline{b}}(E)=\int_{E} e^{\phi_{n}\left(\underline{b} x_{n}^{\infty}\right)-\phi_{n}\left(\underline{a} x_{n}^{\infty}\right)} \mathrm{d} m(x) ; \\
& m \circ v_{\underline{a}, \underline{b}}(E)=\int_{E} e^{\widetilde{\phi}_{n}\left(\underline{b} x_{n}^{\infty}\right)-\widetilde{\phi}_{n}\left(\underline{a}_{n}^{\infty}\right)} \mathrm{d} m(x),
\end{aligned}
$$

para cada $E \in \mathcal{B} \cap[\underline{a}]$. Das Equações (2.69), (2.70) e (2.71) obtemos

$$
\begin{aligned}
\int_{E} e^{\phi_{n}\left(\underline{b} x_{n}^{\infty}\right)-\phi_{n}\left(\underline{a} x_{n}^{\infty}\right)} \mathrm{d} m(x) & =\int_{E} e^{\widetilde{\phi}_{n}\left(\underline{b} x_{n}^{\infty}\right)-\widetilde{\phi}_{n}\left(\underline{a} x_{n}^{\infty}\right)} \mathrm{d} m(x) \\
& =\int_{E} e^{\phi_{n}\left(\underline{b} x_{n}^{\infty}\right)-\phi_{n}\left(\underline{a} x_{n}^{\infty}\right)+h\left(\underline{b} x_{n}^{\infty}\right)-h\left(\underline{a} x_{n}^{\infty}\right)} \mathrm{d} m(x) \\
& =\int_{E} e^{\phi_{n}\left(\underline{b} x_{n}^{\infty}\right)-\phi_{n}\left(\underline{a} x_{n}^{\infty}\right)} \frac{h\left(\underline{b} x_{n}^{\infty}\right)}{h\left(\underline{a} x_{n}^{\infty}\right)} \mathrm{d} m(x) .
\end{aligned}
$$


Portanto

$$
\int_{E} e^{\phi_{n}\left(\underline{b} x_{n}^{\infty}\right)-\phi_{n}\left(\underline{a} x_{n}^{\infty}\right)}\left(1-\frac{h\left(\underline{b} x_{n}^{\infty}\right)}{h\left(\underline{a} x_{n}^{\infty}\right)}\right) \mathrm{d} m(x)=0,
$$

para todo Boreliano $E \in \mathcal{B} \cap[\underline{a}]$. Como $h$ é uma função contínua temos que $h\left(\underline{a} x_{n}^{\infty}\right)=h\left(\underline{b} x_{n}^{\infty}\right)$ para cada $x \in[\underline{a}]$ tal que $a_{n-1}=b_{n-1}=c$.

Notemos que $\underline{a}$ e $\underline{b}$ foram escolhidos de forma arbitrária tal que $a_{n-1}=b_{n-1}=c,\left.\log h\right|_{\pi_{n}^{-1}\{c\}}$ só depende das coordenadas $x_{k}$, para $k \geq n$, logo é $\left.\sigma^{-n} \mathcal{B}\right|_{\pi_{n}^{-1}\{c\}}$-mensurável, como $c \in S$ é arbitrário, pelo Lema 92 temos que $h$ é $\sigma^{-n} \mathcal{B}$-mensurável. Como $n \geq 2$ é arbitrário temos que $h$ é $\sigma^{-n} \mathcal{B}$-mensurável para cada $n \in \mathbb{N}$ logo $h$ é mensurável em relação à $\sigma$-álgebra caudal $\cap_{n \geq 1} \sigma^{-n} \mathcal{B}$.

Pelo Teorema 2.5 em [Sa5] temos que $\nu$ é uma medida exata logo $h(x)$ é $\nu$-constante para cada $x \in \Sigma_{A}$.

Agora mostraremos ii.) $\Rightarrow$ i.). Para cada $E \in \mathcal{B}$ temos que $m(E)=\alpha \nu(E)$ isto é $m=\alpha \nu$ logo $m$ é uma medida $(\phi, \lambda)$-conforme. Como $m$ é uma medida $\sigma$-invariante temos que

$$
m\left(\pi_{n}^{-1}\{a\}\right)=m([a])<\infty, \quad \forall a \in S, n \in \mathbb{N} .
$$

Logo a sub- $\sigma$-álgebra $\sigma^{-n} \mathcal{B}$ é compatível com a esperança condicional para cada $n \geq 1$, assim pela Proposição 78 e Corolário 85 temos que $m$ é uma medida $\phi-$ DLR.

O seguinte teorema mostra as relações entre as medidas DLR e de equilíbrio para o shift satisfazendo a condição BIP.

Teorema 96. Sejam $\Sigma_{A}$ um shift de Markov topologicamente mixing com a propriedade BIP e $\phi: \Sigma_{A} \rightarrow \mathbb{R}$ um potencial que satisfaz a condição de Walters com $\operatorname{Var}_{1} \phi<\infty$ e $P_{G}(\phi)<\infty$. Então:

i.) $\{$ medida $\phi-$ DLR $\sigma-$ inavariante $\} \cap\left\{h_{(\cdot)}(\sigma)<\infty\right\} \subset\{$ medida $\phi-$ Equilibrio $\}$;

ii.) $\{$ medida $\phi-E q u i l i ́ b r i o\} \subset\{$ medidas $\widetilde{\phi}-\mathrm{DLR} \sigma-$ invariante $\}$,

onde $\widetilde{\phi}=\phi+\log h-\log h \circ \sigma-P_{G}(\phi)$.

Demonstração. Nas hipóteses do teorema temos que $\sup \phi<\infty$, veja o Lema 4 em [BMP], logo a existência da medida de equilíbrio para o potencial $\phi$ é garantida, pelo Teorema $1.2 \mathrm{em}[\mathrm{BS}]$ temos que esta é dada por $m=h \mathrm{~d} \nu$ onde $\nu$ é uma medida finita tal que $L_{\phi}^{*} \nu=\lambda \nu$. A seguir provaremos o item $i$.). Consideremos $\mu$ uma medida $\phi-\operatorname{DLR} \sigma$-invariante. Sejam $n \in \mathbb{N}$ e $[\underline{a}]$ um cilindro de tamanho $n$. Sejam $H_{1}, H_{2}>0$ as constantes positivas tal que $H_{1}<h(x)<H_{2}$ para cada $x \in \Sigma_{A}$ e $M:=\sup _{n \geq 1} \operatorname{Var}_{n+1} \phi_{n}+\operatorname{Var}_{1} \phi$. Pela demonstração do Teorema 93 temos

$$
\lambda^{-n} e^{\phi_{n}\left(\underline{a} \sigma^{n} x\right)} \mathbb{1}_{\left\{\underline{a} \sigma^{n} x \in \Sigma_{A}\right\}} \leq \frac{e^{M}}{H_{1} K} m([\underline{a}]), \quad \forall x \in \Sigma_{A},
$$

onde $K>0$ é dado por (2.62).

Das Equações (2.64) e (2.73) obtemos que dado $n \in \mathbb{N}$, e $[\underline{a}]$ um cilindro de tamanho $n$ então

$$
\frac{e^{\phi_{n}\left(\underline{a} \sigma^{n} x\right)} \mathbb{1}_{\left\{\underline{a} \sigma^{n} x \in \Sigma_{A}\right\}}}{\sum_{\sigma^{n} y=\sigma^{n} x} e^{\phi_{n}(y)}} \leq \frac{e^{M}}{H_{2} K} m([\underline{a}]), \quad \forall x \in \Sigma_{A},
$$

como $\mu$ é uma medida $\phi-D L R$ obtemos que $\mathbb{E}_{\mu}\left[\mathbb{1}_{[\underline{a}]} \mid \sigma^{-n} \mathcal{B}\right](x) \leq C m[\underline{a}]$, onde $C=\frac{e^{M}}{H_{2} K}$. Portanto $\mu[a] \leq C m[a]$ para todo cilindro de tamanho $n$, desde que $n$ é arbitrário obtemos que $\mu \leq C m$ é satisfeito para qualquer cilindro, logo

$$
\mu \ll m,
$$


como $m$ é uma medida ergódica, veja o Teorema 4.7 em [Sa5], concluímos que $\mu=m=h \mathrm{~d} \nu$.

Para mostrar o item ii.) notemos que a medida de equilíbrio para o potencial $\phi$ é dada por $m=h \mathrm{~d} \nu$. Além disso um calculo padrão mostra que $L_{\widetilde{\phi}}^{*} m=m$ logo $m$ é uma medida $\widetilde{\phi}$-conforme e portanto $\widetilde{\phi}-\mathrm{DLR}$.

Observação 97. Notemos que a Proposição 95 garante que em geral não é possível que uma medida de equilíbrio para um potencial $\phi$ seja DLR para o mesmo potencial. Salvo que este normalize o operador de Ruelle, nesse sentido é esperado mostrar que a medida de equilíbrio para o potencial seja DLR para seu potencial normalizado como indica o item ii.) do Teorema acima.

Observação 98. Vale a pena mencionar que o item ii.) é valido em condições mais gerais como mostra a Proposição 86. 



\section{Capítulo 3}

\section{Transições de Fase Tipo Volume em Shifts de Markov}

O resultado principal deste capítulo é o Teorema 105 que mostra a existência de transições de fase tipo volume na família de automedidas $\left\{\nu_{\beta}\right\}_{\beta>0}$ associada a família de potenciais $\{\beta \phi\}_{\beta>0}$, isto mostra um bom comportamento do shift renewal em relação à finitude da automedida. Nos trabalhos de G. Iommi e O. Sarig mostra-se que a família de automedidas $\left\{\nu_{\beta}\right\}_{\beta>0}$ associada à família de potencias $\{\beta \phi\}_{\beta>0}$ também tem um bom comportamento em relação à transição de fase nos modos de recorrência e a existência de medidas maximizantes. A seguir estes resultados serão brevemente apresentados , para mais detalhes veja [Io] e [Sa3], e finalmente o resultado principal deste capítulo.

O. Sarig em [Sa3] mostra que existem shifts topologicamente mixing e potenciais $\phi(x)=$ $\phi\left(x_{0}, x_{1}\right)$ tais que:

i.) Para alguma sequência $\beta_{n} \downarrow 0, \beta \phi$ é recorrente em $\left(\beta_{i+1}, \beta_{i}\right)$ para $i$ par e transiente para $i$ impar.

ii.) Existem $\beta_{1}$ e $\beta_{2}$ tais que $1<\beta_{1}<\beta_{2}<\infty$, $\beta \phi$ é nulamente recorrente para $\beta \in\left(1, \beta_{1}\right)$, positivamente recorrente para $\beta \in\left(\beta_{1}, \beta_{2}\right)$ e transiente para $\beta>\beta_{2}$.

Isso mostra que a família de potenciais $\beta \phi$, para $\beta>0$, pode não ter um bom comportamento em relação aos modos de recorrência. Porém, para o shift renewal, existe um único $\beta_{c} \in(0, \infty]$ tal que $\beta \phi$ é positivamente recorrente para cada $\beta<\beta_{c}$ e transiente para $\beta>\beta_{c}$. O valor $\beta_{c}$ é chamado de valor crítico, e é onde $\beta \phi$ muda o modo de recorrência, neste sentido é entendido esta transição de fase. Vale o seguinte teorema:

Teorema 99 (Sarig [Sa3]). Sejam $\Sigma_{A}$ o shift renewal e $\phi: \Sigma_{A} \rightarrow \mathbb{R}$ um potencial localmente Hölder tal que $\sup \phi<\infty$. Então existe $0<\beta_{c} \leq \infty$ tal que:

i.) Para cada $\beta \in\left(0, \beta_{c}\right)$ o potencial $\beta \phi$ é positivamente recorrente e para cada $\beta>\beta_{c}$ é transiente.

ii.) $P_{G}(\beta \phi)$ é analítica em $\beta \in\left(0, \beta_{c}\right)$ e linear em $\left(\beta_{c}, \infty\right)$. Além disso, $P_{G}(\beta \phi)$ é contínua em $\beta_{c}$ mas não analítica.

Para os detalhes da prova veja o Teorema 5 em [Sa3]. O Teorema 99 também é válido para os shifts na classe $\Sigma_{R}{ }^{1}$. Isto é devido ao fato que nestes shifts de Markov para cada $n \in \mathbb{N}$ existe no máximo um ponto periódico com período mínimo $n$ tal que todas as letras da palavra de tamanho $n$ que define o período são diferentes.

\footnotetext{
${ }^{1}$ Veja a definição na Observação 6.
} 
Observação 100. Definamos $M$ como o coeficiente angular da parte linear da função $P_{G}(\beta \phi)$. Pelo Teorema do discriminante, Teorema 2 em [Sa3], temos que $P_{G}(\beta \phi)>M \beta$ para todo $\beta<\beta_{c}$ e $P_{G}(\beta \phi)=M \beta$ para $\beta>\beta_{c}$.

Note que o potencial $\beta_{c} \phi$ não tem um modo de recorrência definido como mostra O. Sarig em [Sa3]. Para isso considere $\left\{f_{n}\right\}_{n \geq 1}$ uma sequência de números tais que $f_{n}>0$ e $\log \left(f_{n}\right)=O(n)$, definamos

$$
\phi:=\sum_{n \geq 1} \mathbb{1}_{[1, n]} \log \left(f_{n}\right) \quad \text { e } \quad \zeta(s):=\sum_{n \geq 1} n^{-s},
$$

$\log \beta_{c} \phi$ é:

i.) positivamente recorrente para $f_{n}=\frac{1}{\zeta(3) n^{3}}$.

ii.) nulamente recorrente para $f_{n}=\frac{1}{\zeta(2) n^{2}}$.

iii.) transiente para $f_{n}=\frac{C}{n[\log (2 n)]^{2}}$, onde $C$ é uma constante tal que $\sum_{n \geq 1} f_{n}=\frac{1}{2}$.

Consideremos

$$
m(\phi):=\sup _{\nu \in \mathcal{M}_{\sigma}^{1}\left(\Sigma_{A}\right)}\left\{\int \phi \mathrm{d} \nu\right\},
$$

uma medida $\mu_{\phi} \in \mathcal{M}_{\sigma}^{1}\left(\Sigma_{A}\right)$ é chamada de $\phi$-maximizante se

$$
m(\phi)=\int \phi \mathrm{d} \mu_{\phi}
$$

O seguinte teorema dado por G. Iommi fornece mais informações sobre shifts renewal em relação as medidas $\phi$-maximizantes.

Teorema 101 (Iommi [Io]). Sejam $\Sigma_{A}$ um shift na classe $\mathcal{R}$ e $\phi: \Sigma_{A} \rightarrow \mathbb{R}$ um potencial localmente Hölder tal que $\sup \phi<\infty$. Logo:

i.) Se $\beta_{c}=\infty$, então existe uma medida $\phi$-maximizante.

ii.) Se $\beta_{c}<\infty$, então não existe uma medida $\phi$-maximizante e $m(\phi)=M$, onde $M$ é o coeficiente angular da parte linear da função $P_{G}(\beta \phi)$.

Lema 102. Sejam $\Sigma_{A}$ o shift renewal e $\mathcal{O}^{n}(1):=\left\{x \in \Sigma_{A} \mid \sigma^{n} x=x, x_{0}=1\right\}$ para cada $n \in \mathbb{N}$. Então $\left|\mathcal{O}^{n}(1)\right|=2^{n-1}$.

Demonstração. É fácil ver que $\left|\mathcal{O}^{1}(1)\right|=1$. Fixemos $n \in \mathbb{N}$, suponhamos que $\left|\mathcal{O}^{n}(1)\right|=2^{n-1}$, a seguir mostraremos que $\left|\mathcal{O}^{n+1}(1)\right|=2^{n}$.

Note que $(1 \underline{\omega})_{p e r},(\underline{\omega} 1)_{p e r} \in \mathcal{O}^{n+1}(1)$ para cada $\underline{\omega} \in \mathcal{O}^{n}(1)$, logo

$$
\left|\mathcal{O}^{n+1}(1)\right|=2\left|\mathcal{O}^{n}(1)\right|=2^{n} .
$$

Notemos que para cada $\beta>0, P_{G}(\beta \phi)<\infty$. De fato

$$
\begin{aligned}
P_{G}(\beta \phi) & =\lim _{n \rightarrow \infty} \frac{1}{n} \log \left(\sum_{\sigma^{n} x=x} e^{\beta \phi_{n}(x)} \mathbb{1}_{[1]}(x)\right) \\
& \leq \lim _{n \rightarrow \infty} \frac{1}{n} \log \left(\sum_{\sigma^{n} x=x} e^{n \beta \sup \phi_{1}} \mathbb{1}_{[1]}(x)\right)
\end{aligned}
$$




$$
\begin{aligned}
& =\beta \sup \phi+\lim _{n \rightarrow \infty} \frac{1}{n} \log \left(\sum_{\sigma^{n} x=x} \mathbb{1}_{[1]}(x)\right) \\
& =\beta \sup \phi+\lim _{n \rightarrow \infty} \frac{1}{n} \log \left(\#\left\{x \in \Sigma_{A} \mid \sigma^{n} x=x, x_{0}=1\right\}\right) \\
& =\beta \sup \phi+\lim _{n \rightarrow \infty} \frac{1}{n} \log 2^{n-1} \\
& =\beta \sup \phi+\log 2<\infty .
\end{aligned}
$$

Para cada $\beta \in\left(0, \beta_{c}\right)$ consideremos $\mu_{\beta}$ a medida de RPF associada ao potencial $\beta \phi$. O seguinte lema será importante na demonstração da Proposição 104.

Lema 103. Sejam $\Sigma_{A}$ o shift renewal e $\phi: \Sigma_{A} \rightarrow \mathbb{R}$ um potencial localmente Hölder tal que $\sup \phi<\infty$. Então

$$
\frac{\mathrm{d}}{\mathrm{d} \beta} P_{G}(\beta \phi)=\int \phi \mathrm{d} \mu_{\beta}
$$

para cada $\beta \in\left(0, \beta_{c}\right)$.

Demonstração. A prova é análoga ao Proposição 2.6.13 em [MaUr2].

Para cada $\beta \in\left(0, \beta_{c}\right)$ definamos a função

$$
\psi(\beta):=\frac{P_{G}(\beta \phi)}{\beta} .
$$

Proposição 104. Sejam $\Sigma_{A}$ o shift renewal e $\phi: \Sigma_{A} \rightarrow \mathbb{R}$ um potencial localmente Hölder tal que $\sup \phi<\infty$. Logo a função $\psi$ é

i.) contínua;

ii.) decrescente.

Além disso,

iii.) $\lim _{\beta \rightarrow 0^{+}} \psi(\beta)=+\infty$.

Demonstração. O item $i$.) é uma consequência direta do Teorema 99 item ii.). A seguir mostraremos que a função $\psi$ é decrescente em $\left(0, \beta_{c}\right)$.

Seja $\mu_{\beta}$ a única medida de equilíbrio associada ao potencial $\beta \phi$ para $\beta \in\left(0, \beta_{c}\right)$. Definamos a função $\varphi:\left(0, \beta_{c}\right) \rightarrow \mathbb{R}$ dada por

$$
\varphi(\beta):=P_{G}(\beta \phi)-\beta m(\phi)
$$

Primeiro mostraremos que $\varphi$ é uma função positiva. Quando $\beta_{c}<\infty$ temos que essa afirmação é direta, veja a Observação 100 e o Teorema 101 item $i$.). Consideremos $\beta_{c}=+\infty$, pelo Teorema 101 item $i$.) existe uma medida $\mu_{\phi} \in \mathcal{M}_{\sigma}^{1}\left(\Sigma_{A}\right)$ que é $\phi$-maximizante. Neste caso

$$
\varphi(\beta) \geq h_{\mu_{\phi}}(\sigma)+\beta \int \phi \mathrm{d} \mu_{\phi}-\beta m(\phi)=h_{\mu_{\phi}}(\sigma) \geq 0,
$$

$\log 0$

$$
\varphi \geq 0, \quad \text { para cada } \beta>0 .
$$

Usando o Lema 103 obtemos

$$
\frac{\mathrm{d}}{\mathrm{d} \beta} \varphi(\beta)=\int \phi \mathrm{d} \mu_{\beta}-m(\phi) \leq 0,
$$


portanto $\varphi$ é uma função convexa e não-crescente.

Suponhamos por contradição que existe $\tilde{\beta}>0$ satisfazendo $P_{G}(\tilde{\beta} \phi)=\tilde{\beta} m(\phi), \operatorname{logo}$ :

$$
P_{G}(\beta \phi)=\beta m(\phi), \quad \text { para todo } \beta \in[\tilde{\beta}, \infty),
$$

e da Equação (3.3) temos que cada medida $\mu_{\beta}$ é $\phi$-maximizante para $\beta>\tilde{\beta}$.

Para cada $\beta>\tilde{\beta}$ temos

$$
\beta m(\phi)=P_{G}(\beta \phi)=h_{\mu_{\beta}}(\sigma)+\beta \int \phi \mathrm{d} \mu_{\beta},
$$

$\operatorname{logo} h_{\mu_{\beta}}(\sigma)=0, \forall \beta>\tilde{\beta}$. De forma análoga pode-se mostrar que $h_{\mu_{\phi}}(\sigma)=0$.

Fixado $\beta>\tilde{\beta}$ temos

$$
\begin{aligned}
P_{G}(\beta \phi) & =h_{\mu_{\beta}}(\sigma)+\beta \int \phi \mathrm{d} \mu_{\beta} ; \\
& =h_{\mu_{\phi}}(\sigma)+\beta \int \phi \mathrm{d} \mu_{\phi},
\end{aligned}
$$

logo as medida $\mu_{\phi}$ e $\mu_{\beta}$ são medidas de equilíbrio associadas ao potencial $\beta \phi$, como a medida de equilíbrio é única, temos que $\mu_{\phi}=\mu_{\beta}$. Como $\beta$ foi escolhido de maneira arbitrária, temos que $\mu_{\phi}=\mu_{\beta}$ para todo $\beta>\tilde{\beta}$, note que isso também demonstra que existe uma única medida $\phi$-maximizante.

Consideremos $\beta_{1}, \beta_{2} \in(\tilde{\beta},+\infty)$, como $\beta_{1} \phi$ e $\beta_{2} \phi$ tem a mesma medida de equilíbrio logo $\beta_{1} \phi \sim \beta_{2} \phi+c$, veja o Teorema 57, onde $c$ é alguma constante. Pela Proposição 30 item iii.) e a Equação (3.4) temos que

$$
c=\left(\beta_{1}-\beta_{2}\right) m(\phi) .
$$

Consideremos $\bar{x}=(1,1,1, \ldots) \in \Sigma_{A}$. Como $\beta_{1} \phi \sim \beta_{2} \phi+c$, temos que existe uma função $\alpha$ tal que $\beta_{1} \phi=\beta_{2} \phi+\alpha-\alpha \circ \sigma+c, \log \mathrm{O}$

$$
c=\left(\beta_{1}-\beta_{2}\right) \phi(\bar{x})
$$

De (3.5) e (3.6) temos que

$$
m(\phi)=\phi(\bar{x})=\int \phi \mathrm{d} \delta_{\bar{x}}
$$

Como a medida maximizante é única temos que $\delta_{\bar{x}}=\mu_{\phi}$, isto é uma contradição pois a medida de equilíbrio quando existe vem do operador de Ruelle e dá massa positiva a cada cilindro. Portanto $\varphi>0$.

Como $\phi$ é uma função positiva e não-crescente, para cada $\beta_{1}, \beta_{2} \in\left(0, \beta_{c}\right)$ tal que $\beta_{1}<\beta_{2}$ temos $\varphi\left(\beta_{1}\right) \geq \varphi\left(\beta_{2}\right)>0$. Assim obtemos

$$
\psi\left(\beta_{1}\right)=\frac{\varphi\left(\beta_{1}\right)}{\beta_{1}}+m(\phi)>\frac{\varphi\left(\beta_{2}\right)}{\beta_{2}}+m(\phi)=\psi\left(\beta_{2}\right),
$$

isso prova o item $i i$.$) .$

A seguir mostraremos o item iii.), isto é,

$$
\lim _{\beta \rightarrow 0^{+}} \psi(\beta)=+\infty
$$

Para todo $0<\beta<\beta_{c}$, temos que $\psi^{\prime}(\beta)=\frac{1}{\beta}\left(P_{G}^{\prime}(\beta \phi)-\psi(\beta)\right)$. Como $\psi$ é decrescente, logo

$$
P_{G}^{\prime}(\beta \phi)-\psi(\beta)<0 .
$$


Seja $\beta_{0}<\beta_{c}$, como $P_{G}(\beta \phi)$ é convexa em $\beta$, temos que a reta tangente a $P_{G}(\beta \phi)$ em $\left(\beta_{0}, P_{G}\left(\beta_{0} \phi\right)\right)$ está abaixo da curva $\left(\beta, P_{G}(\beta \phi)\right)$ para $0<\beta<\beta_{c}$, isto é,

$$
P_{G}\left(\beta_{0} \phi\right)+P_{G}^{\prime}\left(\beta_{0} \phi\right) \beta-P_{G}^{\prime}\left(\beta_{0} \phi\right) \beta_{0} \leq P_{G}(\beta \phi),
$$

equivalentemente

$$
P_{G}^{\prime}\left(\beta_{0} \phi\right)+\frac{\beta_{0}}{\beta}\left(\psi\left(\beta_{0}\right)-P_{G}^{\prime}\left(\beta_{0} \phi\right)\right) \leq \psi(\beta),
$$

usando (3.9) e fazendo $\beta \rightarrow 0^{+}$obtemos (3.8).

\subsection{Existência de Transições de fase Tipo Volume}

O Exemplo 37 mostra que para cada $\beta>0$ o potencial $\beta \phi$ é positivamente recorrente, onde $\phi(x):=x_{0}-x_{1}$. Além disso $\nu_{\beta}$ é uma medida finita para cada $\beta \in(0, \log 2)$ e infinita para cada $\beta \geq$ $\log 2$, onde $\nu_{\beta}$ é a automedida que existe pelo Teorema de Ruelle-Perron-Frobenius Generalizado. A seguir mostraremos que a finitude da automedida no shift renewal tem um comportamento de transição de fase análoga ao dos modos de recorrência.

Teorema 105. Sejam $\Sigma_{A}$ o shift renewal e $\phi: \Sigma_{A} \rightarrow \mathbb{R}$ um potencial localmente Hölder tal que $\sup \phi<\infty$. Então existe $\tilde{\beta}_{c} \in\left(0, \beta_{c}\right]$ tal que $\nu_{\beta}$ é uma medida finita para $\beta \in\left(0, \tilde{\beta}_{c}\right)$ e é infinita para $\beta \in\left(\tilde{\beta}_{c}, \beta_{c}\right)$, onde $\nu_{\beta}$ é a automedida associada ao potencial $\beta \phi$.

Demonstração. Para cada $\beta>0$ temos que

$$
P_{G}(\beta \phi) \leq \log 2+\beta \sup \phi,
$$

$\operatorname{logo} P_{G}(\beta \phi)<\infty$ para cada $\beta>0$.

Para cada $\beta \in\left(0, \beta_{c}\right)$ o potencial $\beta \phi$ é positivamente recorrente e assim pelo Teorema Generalizado de Ruelle-Perron-Frobenius existe uma medida $\sigma$-finita $\nu_{\beta}$ tal que

$$
e^{P_{G}(\beta \phi)} \int f \mathrm{~d} \nu_{\beta}=\int L_{\beta \phi} f \mathrm{~d} \nu_{\beta}, \quad \text { para cada } f \in L^{1}\left(\nu_{\beta}\right) .
$$

Para cada $a \geq 2$ consideremos $f:=\mathbb{1}_{[a]}$, logo a Equação (3.10) reduz-se a

$$
e^{P_{G}(\beta \phi)} \nu_{\beta}([a])=\int_{[a-1]} e^{\beta \phi(a x)} \mathrm{d} \nu_{\beta}
$$

Consideremos $\gamma_{a}:=(\overline{a, a-1, \cdots, 1})$, para cada $a \geq 2$, logo

$$
\phi\left(\gamma_{a}\right)-\operatorname{Var}_{a} \phi \leq \phi(a x) \leq \phi\left(\gamma_{a}\right)+\operatorname{Var}_{a} \phi \text {, para cada } x \in \sigma[a] .
$$

Usando a Equação (3.11) na desigualdade anterior obtemos

$$
e^{-\beta \operatorname{Var}_{a} \phi+\beta \phi\left(\gamma_{a}\right)-P_{G}(\beta \phi)} \nu_{\beta}([a-1]) \leq \nu_{\beta}([a]) \leq e^{\beta \operatorname{Var}_{a} \phi+\beta \phi\left(\gamma_{a}\right)-P_{G}(\beta \phi)} \nu_{\beta}([a-1])
$$

Da Desigualdade (3.13) temos

$$
\nu_{\beta}([1])\left(\sum_{n \geq 1} e^{\beta \sum_{j=2}^{n}-\operatorname{Var}_{j} \phi+\phi\left(\gamma_{j}\right)} e^{-(n-1) P_{G}(\beta \phi)}\right) \leq \nu_{\beta}\left(\Sigma_{A}\right),
$$




$$
\nu_{\beta}\left(\Sigma_{A}\right) \leq \nu_{\beta}([1])\left(\sum_{n \geq 1} e^{\beta \sum_{j=2}^{n} \operatorname{Var}_{j} \phi+\phi\left(\gamma_{j}\right)} e^{-(n-1) P_{G}(\beta \phi)}\right) .
$$

Usando o teste da raiz na Desigualdade (3.15) temos que $\nu_{\beta}$ é finita para cada $\beta>0$ tal que

$$
\limsup _{n \rightarrow \infty}\left(e^{\beta \sum_{j=2}^{n} \operatorname{Var}_{j} \phi+\phi\left(\gamma_{j}\right)} e^{-(n-1) P_{G}(\beta \phi)}\right)^{\frac{1}{n}}<1
$$

isto é equivalente a

$$
\limsup _{n \rightarrow \infty}\left[\frac{\beta}{n}\left(\sum_{j=2}^{n} \operatorname{Var}_{j} \phi+\phi\left(\gamma_{j}\right)\right)-\frac{n-1}{n} P_{G}(\beta \phi)\right]<0 .
$$

Usando o fato que $\operatorname{Var}(\phi)<\infty$, temos que

$$
\limsup _{n \rightarrow \infty}\left(\frac{\beta}{n} \sum_{j=2}^{n} \phi\left(\gamma_{j}\right)\right)-P_{G}(\beta \phi)=\limsup _{n \rightarrow \infty}\left[\frac{\beta}{n}\left(\sum_{j=2}^{n} \operatorname{Var}_{j} \phi+\phi\left(\gamma_{j}\right)\right)-\frac{n-1}{n} P_{G}(\beta \phi)\right] .
$$

Logo $\nu_{\beta}$ é finita para cada $0<\beta<\beta_{c}$ tal que

$$
\limsup _{n \rightarrow \infty} \frac{1}{n} \sum_{j=2}^{n} \phi\left(\gamma_{j}\right)<\frac{P_{G}(\beta \phi)}{\beta}
$$

De maneira análoga em (3.14), $\nu_{\beta}$ é infinita para cada $0<\beta<\beta_{c}$ tal que

$$
\limsup _{n \rightarrow \infty} \frac{1}{n} \sum_{j=2}^{n} \phi\left(\gamma_{j}\right)>\frac{P_{G}(\beta \phi)}{\beta} .
$$

Definamos

$$
\tilde{\beta}_{c}:=\sup \left\{\beta \in\left(0, \beta_{c}\right]: \limsup _{n \rightarrow \infty} \frac{1}{n} \sum_{j=2}^{n} \phi\left(\gamma_{j}\right)<\frac{P_{G}(\beta \phi)}{\beta}\right\},
$$

pela Proposição 104 temos que $\tilde{\beta}_{c}$ existe e é único.

Notemos que $\beta_{c}$ e $\tilde{\beta}_{c}$ podem ter valores diferentes:

i.) Seja $\phi(x)=c$, onde $c \in \mathbb{R}$, neste caso $\beta_{c}=\tilde{\beta}_{c}=+\infty$.

ii.) Considerando $\phi(x)=x_{0}-x_{1}$ temos que $\tilde{\beta}_{c}=\log 2$ e $\beta_{c}=+\infty$.

Observação 106. Notemos que o Teorema 105 também é válido para a classe $\Sigma_{R_{1}}{ }^{2}$ e o valor $\tilde{\beta}_{c}$ é dado por (3.16).

Consideremos $\Sigma_{\mathcal{R}^{-}}$o shift de Markov, onde $\mathcal{R}^{-}$é a matriz de transição $\left(A_{i, j}\right)_{\mathbb{N} \times \mathbb{N}}$ com entradas $A(i, i+1), A(i, 1)$ igual a 1 para cada $i \geq 1$ e o resto das entradas igual a zero, isto é representado pelo grafo da Figura 3.1. Para o shift de Markov $\Sigma_{\mathcal{R}^{-}}$, o Teorema de Ruelle-Perron-Frobenius Generalizado garante a existência de automedidas $\nu_{\beta}$ associadas ao potencial $\beta \phi$, para $0<\beta<\beta_{c}$. Além disso, como $\Sigma_{\mathcal{R}^{-}}$é localmente compacto o Teorema 46 garante a existência de automedidas $\nu_{\beta}$ associadas ao potencial $\beta \phi$ para $\beta \geq \beta_{c}$. Como $\Sigma_{\mathcal{R}^{-}} \in \Sigma_{R}$, logo o Teorema 99 é válido para $\Sigma_{\mathcal{R}^{-}}$, assim este shift de Markov tem uma transição de fase no sentido do modo de recorrência. A seguinte proposição garante que $\Sigma_{\mathcal{R}^{-}}$possui potenciais onde não há transição de fase em relação à finitude da medida.

${ }^{2}$ Veja a definição no Exemplo 7. 


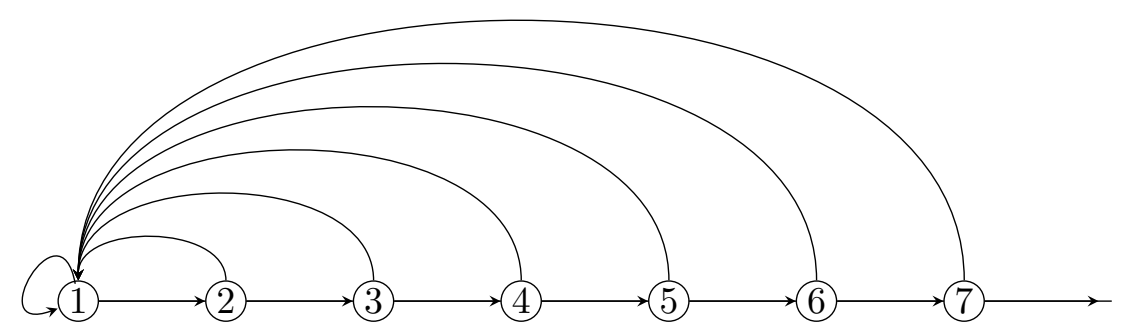

Figura 3.1: Shift $\Sigma_{\mathcal{R}^{-}}$

Proposição 107. Seja $\phi: \Sigma_{\mathcal{R}^{-}} \rightarrow \mathbb{R}$ tais que $\operatorname{Var}_{2} \phi=0$ e $\phi_{n}\left(\gamma_{n}\right)=0$ para cada $n \geq 1$, onde $\gamma_{n}=(\overline{1,2, \ldots, n}) . S e$

$$
\limsup _{n \rightarrow \infty} \frac{\phi(n, 1)}{n} \neq 0,
$$

então $\nu_{\beta}$ é finita ou infinita para todo $\beta>0$.

Demonstração. Note que para cada $\beta>0$, temos que

$$
P_{G}(\beta \phi)=\log 2 .
$$

e o potencial $\beta \phi$ é positivamente recorrente. Assim, pelo Teorema de Ruelle-Perron-Frobenius Generalizado, existe uma medida $\sigma$-finita $\nu_{\beta}$ associada ao potencial $\beta \phi$, para cada $\beta>0$, satisfazendo

$$
2 \int f \mathrm{~d} \nu_{\beta}=\int L_{\beta \phi} f \mathrm{~d} \nu_{\beta}, \quad \text { for every } f \in L^{1}\left(\nu_{\beta}\right) .
$$

Usando a Equação (3.19) e o fato que $\phi_{n}\left(\gamma_{n}\right)=0$ para todo $n \in \mathbb{N}$, obtemos

$$
\nu_{\beta}([n])=e^{\beta \phi(n, 1)} \nu_{\beta}([1]), \quad \text { para } n \geq 2 .
$$

Logo

$$
\nu_{\beta}\left(\Sigma_{\mathcal{R}^{-}}\right)=\nu_{\beta}([1]) \sum_{n \geq 1} e^{\beta \phi(n, 1)} .
$$

Portanto $\nu_{\beta}$ é finita, para cada $\beta>0$, se

$$
\limsup _{n \rightarrow \infty} \frac{\phi(n, 1)}{n}<0 .
$$

Analogamente, $\nu_{\beta}$ é infinita, para cada $\beta>0$, se

$$
\limsup _{n \rightarrow \infty} \frac{\phi(n, 1)}{n}>0 .
$$

A seguir apresentamos dois potenciais satisfazendo as hipóteses da proposição acima:

i.) Considerando $\phi(x)=c$, onde $c \in \mathbb{R}$. Da Equação (3.20) temos que $\nu_{\beta}\left(\Sigma_{\mathcal{R}^{-}}\right)=+\infty$ para cada $\beta>0$.

ii.) Considerando $\phi(x)=x_{1}-x_{0}$, pode-se ver facilmente da Equação (3.20) que $\nu_{\beta}\left(\Sigma_{\mathcal{R}^{-}}\right)<\infty$ para cada $\beta>0$. 
A seguir temos um potencial que não satisfaz a condição (3.17) e tem uma transição de fase diferente a que acontece no shift renewal.

Exemplo 108. Consideremos $\phi: \Sigma_{\mathcal{R}^{-}} \rightarrow \mathbb{R}$ dado por $\phi(x)=\log \frac{x_{1}}{x_{0}}$. Note que $\lim \sup _{n \rightarrow \infty} \frac{\phi(n, 1)}{n}=$ 0. Pela Equação (3.20), temos

$$
\nu_{\beta}\left(\Sigma_{\mathcal{R}^{-}}\right)=\nu_{\beta}([1]) \sum_{n \geq 1} \frac{1}{n^{\beta}},
$$

portanto $\nu_{\beta}\left(\Sigma_{\mathcal{R}^{-}}\right)$é infinito para $\beta \leq 1$ e finito quando $\beta>1$. 


\section{Conclusões e Questões Futuras}

No caso quando $\Sigma_{A}$ é um shift topologicamente mixing satisfazendo a condição $B I P$ e $\phi$ é um potencial satisfazendo a propriedade de Walters com $P_{G}(\phi)<\infty$ e $\sum_{n>1} \operatorname{Var}_{n} \phi<\infty$ temos que as medidas conformes e DLR são equivalentes. Além disso a medida DLR existe e é única, veja o Teorema 93. A importância desse resultado é que exibe uma classe de shifts de Markov onde as medidas conformes e DLR são equivalentes, para o caso de alfabeto enumerável com cardinalidade infinita. Esse resultado generaliza os resultados para o caso de alfabeto finito dado por O. Sarig e L. Cioletti - A. Lopes em [[CiLo], Teorema B] e [[Sa5], Teorema 3.6], respectivamente. Além disso, nas mesmas condições pode-se concluir que toda medida DLR invariante com entropia métrica finita é de equilíbrio, veja o Teorema 96 , esse resultado é análogo ao obtido por S. Muir para o caso $\mathbb{N}^{d}$ em [Mu1].

A seguir mostramos que a medida DLR é uma propriedade que depende da matriz e não podem ser caracterizadas pelos modos de recorrência como acontece no caso das medidas conformes, veja o Teorema 36. Consideremos o shift de Markov dada pela Figura 3.2, no Exemplo 94 define se uma

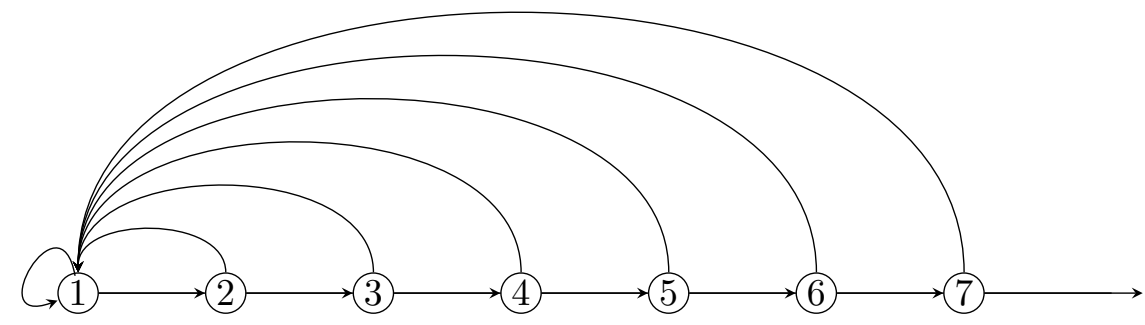

Figura 3.2

medida $\mu$ tal que para qualquer potencial $\phi$ temos que $\mu$ é $\phi-D L R$, onde $\mu([1])=1$ e $\mu([a])=0$ para $a>1$. Além disso mostra se que $\mu$ não é $(\phi, \lambda)$-conforme para qualquer $\lambda>0$. Notemos que a medida $\mu$ não depende do potencial ou de alguma regularidade a ser considerada, só depende do comportamento da matriz.

A seguir mostramos que as medidas DLR não podem ser caracterizadas pelos modos de recorrência. Note que pode se definir potenciais com alguma regularidade tal que exista uma medida conforme, e portanto DLR, diferente a $\mu$; veja os Teoremas 36 e 46. Consideremos os seguintes casos:

a.) [Potencial Recorrente.] Consideremos o potencial $\phi(x)=2 \log \frac{x_{1}}{x_{0}}, \log 0 \phi$ satisfaz a condição de Walters e $P_{G}(\phi)=2$. Notemos que a serie

$$
\sum_{n \geq 1} e^{-n P_{G}(\phi)} Z_{n}(\phi, 1)=+\infty
$$

portanto $\phi$ é recorrente. Assim pelo Teorema Generalizado de Ruelle-Perron-Frobenius temos que existe uma medida conforme $\nu$ que dá massa positiva a cada cilindro. Como $\left\|L_{\phi} \mathbb{1}\right\|_{\infty}<\infty$, 
pela Proposição 78 e Corolário 85 temos que $\nu$ é uma medida DLR no entanto $\nu \neq \mu$ pois $\mu([a])=0$ para cada $a>1$.

b.) [Potencial $\lambda$-Transiente.] Consideremos o potencial $\phi$ do item $a$.) e $\lambda \in \mathbb{R}$. Temos que

$$
\sum_{n \geq 1} \lambda^{-n} Z_{n}(\phi, 1)<\infty
$$

para cada $\lambda>2$. Pelo Teorema 46 existe uma medida $\lambda$-conforme, para cada $\lambda>2$, de maneira análoga ao item a.) esta medida é DLR e diferente a $\mu$.

c.) [Potencial Transiente.] Consideremos o potencial

$$
\phi(x)=\sum_{n \geq 1} \mathbb{1}_{[n, 1]}(x) \log \frac{1}{\zeta(3) n^{3}},
$$

onde $\zeta(s):=\sum_{n \geq 1} n^{-s}$, notemos que $\phi$ satisfaz a condição de Walters. Um cálculo simples mostra que $\beta_{c}=1$, onde $\beta_{c}$ é o valor da transição de fase do modo de recorrência; veja o Capitulo 3. Para cada $\beta>1$, temos que o potencial $\beta \phi$ é transiente e existe uma medida conforme $\nu_{\beta \phi}$. Como $\left\|L_{\beta \phi} \mathbb{1}\right\|_{\infty}<\infty$ temos que $\nu_{\beta \phi}$ é uma medida DLR para cada $\beta>1$, porém $\mu \neq \nu_{\beta \phi}$.

Os itens $a.), b$. ) e $c$.) mostram que as medidas DLR não podem ser caracterizadas pelos modos de recorrência. Além disso, que a família das medidas DLR é maior que as medidas conformes.

A noção de medida DLR pode ser estendida para o caso $\sigma$-finito, veja a Subseção 2.2.2. Além disso mostramos que cada medida conforme $\sigma$-finita é DLR, veja o Corolário 85, para isso exigimos uma regularidade no potencial. Esse corolário generaliza o resultado dado por O. Sarig para medidas finitas, veja a Proposição $2.2 \mathrm{em}$ [Sa5]. Também estudamos a inclusão contraria, assim obtemos o Teorema 89, este caracteriza quando uma medida DLR $\sigma$-finita e $\sigma$-invariante é conforme.

Os Teoremas 36 e 99 garantem a existência da medida conforme $\sigma$-finita $\nu_{\beta}$ associada ao potencial $\beta \phi$ no shift renewal para $\beta \in\left(0, \beta_{c}\right)$, onde $\beta_{c}$ é o máximo valor tal que o potencial $\beta \phi$ é positivamente recorrente para $\beta<\beta_{c}$. O Teorema 105 mostra a existência de um valor $\tilde{\beta}_{c} \in\left(0, \beta_{c}\right]$ tal a medida $\nu_{\beta}$ é finita para cada $\beta \in\left(0, \tilde{\beta}_{c}\right)$ e infinita para $\beta \in\left(\tilde{\beta}_{c}, \beta_{c}\right)$, mostrando assim uma transição de fase tipo volume na família de medidas conformes $\left\{\nu_{\beta}: \beta \in\left(0, \beta_{c}\right)\right\}$ e a existência de medidas conformes, portanto DLR, finitas para altas temperaturas.

É possível mostrar um teorema geral de transição de fase tipo volume para o shift $\mathcal{R}^{-}$?. Estender e estudar medidas DLR infinitas (mas $\sigma$-finitas) em espaços multidimensionais como $\mathbb{N}^{d}$, seguindo S. Muir [Mu1], por exemplo? 


\section{Referências}

[Aar] J. Aaronson. An Introduction to Infinite Ergodic Theory, volume 50 of Mathematical Surveys and Monographs. American Mathematical Society, Providence, RI, 1997. 12

[BMP] R. Bissacot, J. Mengue and E. PÉrez. A Large Deviation Principle for Gibbs States on Markov Shifts at Zero Temperature. ArXiv:1612.05831, preprint, 2016, to appear. 62

[Bo74] R. Bowen. Some systems with unique equilibrium states. Theory of Computing Systems, 8, No. 3, 193-202, 1974. 21, 32

[Bo75] R. Bowen. Equilibrium states and the ergodic theory of Anosov diffeomorphisms. Lecture Notes in Mathematics, vol. 470. Springer, Berlin, 1975. 1

[BS] J. BuZZI AND O.M. SARIG. Uniqueness of equilibrium measures for countable markov shifts and multidimensional piecewise expanding maps. Ergodic Theory and Dynamical Systems, 23, No. 05, 1383-1400, 2003. 32, 62

[Cyr1] V. T. Cyr. PhD thesis, The Pennsylvania States University, 2010. 4, 21, 24, 28

[Cyr2] V. T. Cyr. Transient Markov shifts. Proceedings of the London Mathematical Society, 103, No 6, 923-949, 2011.

[Da] Y. DAOn.Bernoullicity of equilibrium measures on countable Markov shifts. Discrete and Continuous Dynamical Systems, 33, No. 9, 4003-4015, 2013. 4, 30, 32

[DeUr] M. Denker and M. Urbanski. On the existence of conformal measures. Transactions of the American Mathematical Society, 328, No. 2, 563-587, 1991. 9

[Dob] R. Dobrushin. Description of a random field by means of conditional probabilities and conditions for its regularity. Teoriya Veroyatnostei $i$ ee Primeneniya, 13, 201-229, 1968. 1

[FrV] R. Freire AND V. VARGAS. Equilibrium states and zero temperature limit on topologically transitive countable Markov shifts. Transactions of the American Mathematical Society. ArXiv:1511.01527, preprint, 2015, to appear. 22

[Ge] Georgir, H. O. Gibbs measures and phase transitions. No. 9, 2011. 34

[Io] G. Iommi. Ergodic optimization for renewal type shifts. Monatshefte für Mathematik, 150, No. 2, 91-95, 2007. 3, 4, 7, 65, 66

[Kel] G. Keller. Equilibrium States in Ergodic Theory. London Mathematical Society Student Texts 42, Cambridge University Press, Cambridge, 1998. 1, 57

[Ki] B. Kimura. Gibbs Measures on Subshifts. Master's Thesis. University of São Paulo, 2015. 1, 57

[KiTa] J. F. C. Kingman and S. J. TaYlor. Introduction to measure and probability. Cambridge University Press, Cambridge, 1966. 41 
[LaRu] O. LANDFORD AND D. Ruelle. Observables at infinity and states with short range correlation in statistical mechanics. Communications in Mathematical Physics, 13, 194-215, 1969. 1

[CiLo] L. Cioletti And A. Lopes. Interactions, specifications, DLR probabilities and the Ruelle operator in the one-dimensional Lattice. Discrete and Continuous Dynamical Systems , 37, No. 12, 6139-6152, 2017. 1, 33, 34, 35, 58, 73

[MaUr1] R. MaUldin AND M. URBański. Gibbs states on the symbolic space over an infinite alphabet. Israel Journal of Mathematics, 125, No. 1, 93-130, 2001. 19, 21, 34

[MaUr2] R. Mauldin And M. URBański. Graph directed Markov systems: geometry and dynamics of limit sets., Cambridge University Press, Cambridge, 2003. 67

[Mu1] S. MUIR. Gibbs/equilibrium measures for functions of multidimensional shits with countable alphabets. PhD thesis, University of North Texas, 2011. 1, 33, 57, 73, 74

[Mu2] S. MuIR. A New Characterization of Gibbs Measures on $\mathbb{N}^{d}$. Nonlinearity, 24, No. 10, 2933-2952, 2011.

[Pa] S. J. Patterson. The limit set of a Fuchsian group. Acta mathematica, 136, No. 1, 241-273, 1976. 9

[Pe] E. PÉRez Reyes. Principio dos grandes desvios para estados de Gibbs-equilíbrio sobre shift enumeráveis à temperatura zero PhD tesis, Universidad de São Paulo, 2015. 20

[Ru67] D. RuelLe. A variational formulation of equilibrium statistical mechanics and the Gibbs state rule. Commun. Math. Phys. 5, 324-329, 1967. 1

[Ru68] D. Ruelle. Statistical mechanics of a one-dimensional lattice gas. Commun. Math. Phys. 9 (1968), 267-278. 2, 21

[Ru73] D. RuelLe. Statiscal mechanics on a compact set with $Z^{\nu}$ action satisfying expansiveness and specification. Trans. Amer. Math. Soc. 78(6), 988-991, 1972. 30

[Ru76] D. Ruelle. A measure associated with axiom-A attractors. American Journal of Mathematics, 98, No. 3, 619-654, 1976. 12

[Ru04] D. RuELlE. Thermodynamic formalism: the mathematical structure of equilibrium statistical mechanics, 2nd edition. Cambridge University Press, 2004. 1

[Sa1] O. M. SARIG. Thermodynamic formalism for countable Markov shift. Ergodic Theory and Dynamical Systems, 19, No. 6, 1565-1593, 1999. 4, 21, 30

[Sa2] O. M. SARIG. Thermodynamic formalism for null recurrent potentials. Israel Journal of Mathematics, No. 1, 121, 285-311, 2001. 4, 22

[Sa3] O. M. SARIG. Phase transition for countable Markov shifts. Communications in Mathematical Physics, 217, No. 3, 555-577, 2001. 3, 4, 65, 66

[Sa4] O. M. SARIG. Characterization of the existence of Gibbs measure for countable Markov shifts. Proceedings of the American Mathematical Society., 131, No. 6, 1751-1758, 2003. 1, 4, 24, 58

[Sa5] O. M. SARIG. Lecture notes on thermodynamic formalism for topological Markov shifts, Penn State, 2009. 1, 2, 3, 4, 6, 8, 11, 12, 13, 16, 17, 18, 19, 20, 21, 22, 23, 24, 30, 31, 32, 33, 34, 37, $39,44,51,58,60,62,63,73,74$

[Si] Y. Sinai. Gibbs measures in ergodic theory. Russian Mathematical Surveys, 27, 21-69, 1972. 1 
[Sw] O. Shwartz. Thermodynamic Formalism for Transient Potential Functions. ArXiv: 1711.03627v2, preprint, 2018. 4, 21, 24, 28, 60

[Va] V. VArgas. Sobre existência de estados de equilíbrio e limite em temperatura zero para shifts de Markov topologicamente mixing. Tese de Doutorado. Universidade de São Paulo, 2015. 22

[Wal78] P. WALters. Invariant measures and equilibrium states for some mappings which expand distance. Trans. Amer. Math. Soc 236 (1978), 121-153. 8

[Wal20] P. Walters. An introduction to ergodic theory. vol. 79. Springer, Verlag, 2000. 30 



\section{Índice Remissivo}

Medida

de Equilíbrio, 30

de RPF, 23

Conforme, 11

DLR $\sigma$-finita, 43

Operador

de Ruelle, 14

de transferência, 12

Potenciais cohomólogos, 19

Potencial

com variação somável, 8

localmente Hölder, 8

nulamente recorrente, 22

positivamente recorrente, 22

recorrente, 22

satisfazendo a condição de Walters, 8

somável, 8

transient, 22

Pressão de Gurevich, 19

shift de Markov, 5

primitivo, 6

renewal, 7

satisfazendo a condição BIP, 6

topologicamente mixing, 6

transitivo, 6 